Contribution to

XI-th International Symposium on Very High Energy

Cosmic Ray Interactions (Campinas, 17 - 21 July, 2000)

and

Chacaltaya Meeting on Cosmic Ray Physics (La Paz, 23 - 27 July, 2000)

Chacaltaya Cosmic Ray Experiment

(edited by A. Ohsawa)

CERN LIBRARIES, GENEVA

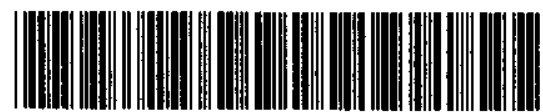

CM-P00042864

(To appear in Nucl. Phys. B and in Nuovo Cim.) 


\section{Content}

"Study of Nuclear Interactions by Emulsion Chambers at Mt. Chacaltaya" ..........

"Hadron/Gamma Identification of Showers Observed by EC's" $\ldots \ldots \ldots \ldots \ldots \ldots \ldots \ldots 20$

"Characteristies of hadron-induced showers observed by the Pamir thick lead chambers"

"A study of the penetrating nature of gamma- and hadron-induced cascade showers

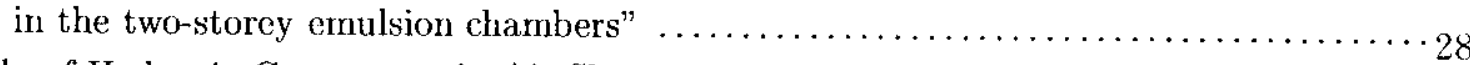

"Study of Hadronic Component in Air Showers at Mt. Chacaltaya"

"On the penetrating showers observed in Chacaltaya two-storey emulsion chambers"

"Study of Nuclear Interactions by Obscrving Families and Air Showers at Mt. Chacaltaya"

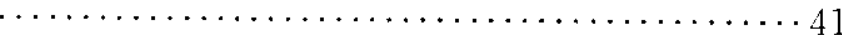

"Nuclear Interactions and Highest Energy Air Showers" $\ldots \ldots \ldots \ldots \ldots \ldots \ldots \ldots \ldots \ldots 46$ 


\title{
Study of Nuclear Interactions by Emulsion Chambers at Mt. Chacaltaya
}

\author{
Akinori Ohsawa \\ ${ }^{a}$ Institute for Cosmic Ray Research, University of Tokyo, Kashiwa, Chiba, 277-8582 Japan.
}

\section{Introduction}

Study of nuclear interactions by observing cosmic rays permits us to investigate what cannot be done by accelerator experiments. Those are the studies of high energy nuclear interactions in the energy region exceeding $1.5 \times 10^{14} \mathrm{eV}$ (CERN $\bar{p} p$ collider of $\sqrt{s}=546 \mathrm{GeV}$ ) or $1.6 \times 10^{15} \mathrm{eV}$ (FNAL Tevatron of $\sqrt{s}=1,800 \mathrm{GeV}$ ), and of exotic events, such as Centauro events[1], etc. which are possibly produced in extremely high energy region and/or by an exotic component among the primary cosmic rays. In this report we discuss high energy nuclear interactions - multiple particle production -- based on the data by cosmicray experiments, leaving important and interesting topics of exotic events elsewhere.

To discuss high energy nuclear interactions it is convenient to start from the energy distribution of produced particles per event, defined as

$\frac{d N}{d E^{*}} \equiv \frac{1}{\sigma_{\text {inel }}} \frac{d \sigma}{d E^{*}}$

because other quantities, such as inelasticity and multiplicity, are derived from it. ${ }^{1}$ Feynman scaling law asserts that the distribution, which is expressed by a variable $x^{*} \equiv 2 p_{\|}^{*} / \sqrt{s}$, is independent of the incident energy of collision as $\sqrt{s} \rightarrow \infty$.[2] The law is verified in the energy region of $\sqrt{s} \leq 63 \mathrm{GeV}$. And one of the empirical formulae of the energy distribution for charyed produced particles is

$\frac{d N}{d x^{*}}=D \frac{\left(1-x^{*}\right)^{d}}{x^{*}}$

with $D=2(d+1) / 3$ and $d=4.0 .[3]$ It leads to the energy distribution in the laboratory system

\footnotetext{
Quantities with and without an asterisk are those in the center of mass system and in the laboratory system, respectively.
}

$$
\frac{d N}{d x}=D \frac{(1-x)^{d}}{x}
$$

because we have $x^{*} \simeq E / E_{0} \equiv x$ at high energies. Then we have the charged multiplicity and the total inelasticity

$m\left(E_{0}\right) \equiv \int_{m_{\pi} / E_{0}}^{1} \frac{d N}{d x} d x \simeq D\left[\ln \left(\frac{E_{0}}{m_{\pi}}\right)-\frac{25}{12}\right]$

and

$<K>\equiv \frac{3}{2} \int_{0}^{1} x \frac{d N}{d x} d x=0.5$

where the factor $3 / 2$ is due to charge independence of produced pions assuming that all the produced particles are pions. Both of the above reproduce the experimental data in low energy region well.(See Fig. 12.)

In Section 2 we discuss violation of the Feynman scaling law in high energies, based on the experimental data by emulsion chamber experiments at Mt. Chacaltaya. And in Section 3 we formulate the energy distribution of produced particles which shows the violation of the Feynman scaling law, based on the data of direct observation by accelerator and cosmic-ray experiments. We discuss the consequences of the formulated distribution at high energies and examine the validity of it using the data of extremely high energy $\left(>10^{18} \mathrm{eV}\right)$ air showers.

\section{Emulsion chamber experiments at Mt. Chacaltaya}

Emulsion chamber experiments have been carried out at Mt. Chacaltaya $(5,200 \mathrm{~m}$, Bolivia) by Brazil-Japan collaboration and by Bolivia-BrazilJapan collaboration, employing various types of the chambers according to the purpose of the study. In this section we present some results by the experiments in order to discuss what the 


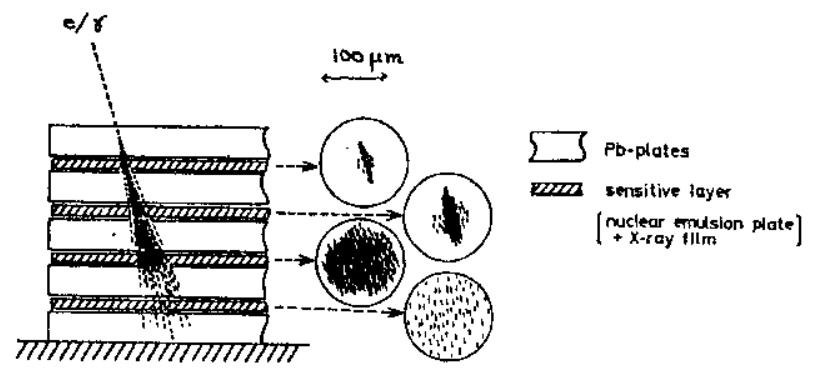

Figure 1. Emulsion chamber consists of lead plates and sensitive layers (X-ray films and/or nuclear emulsion plates), piled up alternately. An electron or a photon, incident upon the chamber produces a cascade shower in the chamber.

Table 1

Performance of the emulsion chamber

\begin{tabular}{l|l|l}
\hline & Nuclear cmulsion plate & \multicolumn{1}{|c}{ X-ray film } \\
\hline Shower position & \multicolumn{1}{|c|}{$\Delta x \sim 10 \mu \mathrm{m}$} & $\Delta x \sim 100 \mu \mathrm{m}$ \\
\hline \multirow{3}{*}{ Shower energy } & electron tracks by microscope & opacity by microphotometer \\
& absolute value & relative value \\
& $\Delta E / E \sim 20 \%$ & $\Delta E / E \sim 10 \%$ \\
& $E_{t h}=0.1 \mathrm{TeV}$ & $E_{t h}=1 \mathrm{TeV}$ \\
\hline Shower structure & observable & impossible \\
\hline
\end{tabular}

experimental data indicate on the nuclear interaction characteristics in high energy region.

\subsection{Emulsion chamber}

Emulsion chamber is a multiple sandwich of lead plates $(1 \mathrm{~cm}$ thick each) and sensitive layers (X-ray films and/or nuclear emulsion plates), piled up alternately.[4] (See Fig. 1.) It detects cascade showers which are produced in the chamber by high energy particles incident upon the chamber. That is, an electron or a photon of high energy, incident upon the chamber, produces a number of clectrons, positrons and photons -.. a cascade shower - in the chamber through a chain of clectromagnetic interactions with lead. The electron component in the cascade shower are recorded by the sensitive layers, and it appears as a small back spot $(\sim 100 \mu \mathrm{m}$ in radius $)$ on the X-ray film after development.

Since a cascade shower develops over several centimeters of lead, the shower spots are found on X-ray films of several successive sensitive laycrs in the chamber. The transition of the spot darkness, which is obtained by a microphotometer with the slit of $200 \times 200 \mu \mathrm{m}^{2}$, along the depth of the chamber enables us to estimate the energy of the incident particle by comparing it with those which are calculated on the basis of the cascade theory[5] and the sensitivity curve of the X-ray film. In this way we can determine the position and the energy of the incident electron or photon, called $(e, \gamma)$ hereafter, with high precision. (See Table 1.) The detection threshold energy of the shower is $E_{t h}=1 \sim 4 \mathrm{TeV}$ on the X-ray film, depending on the experimental conditions.

On the other hand a high energy hadron, incident upon the chamber, causes multiple particle production through a nuclear collision with lead in the chamber. The $\pi^{0}$ 's among the produced particles originate a cascade shower through their decays into $\gamma$-rays. In this way emulsion chamber is sensitive to both $(e, \gamma)$ 's and hadrons, incident 


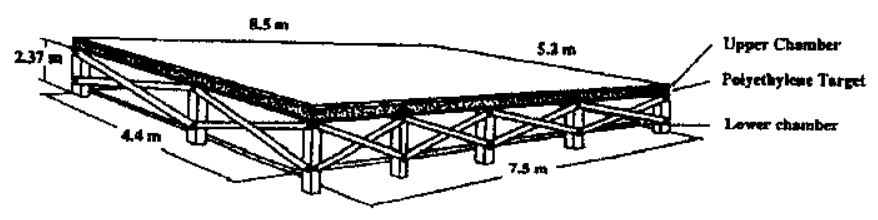

Figure 2. Two-storey chamber, which is used to study C-jets. The chamber consists of upper chamber, target layer, air gap and lower chamber.

Table 2

Structure of two-storey emulsion chambers

\begin{tabular}{|l|cc|cc|cc|}
\hline & \multicolumn{2}{|c|}{ I ('65-'69) } & \multicolumn{2}{c|}{ II ('69-'83) } & \multicolumn{2}{c|}{ III ('83-) } \\
& Area & Thickness & Area & Thickness & Area & Thickness \\
\hline Upper chamber & $6.0 \mathrm{~m}^{2}$ & $6 \mathrm{~cm} \mathrm{~Pb}$ & $44.2 \mathrm{~m}^{2}$ & $6 \mathrm{~cm} \mathrm{~Pb}$ & $44.2 \mathrm{~m}^{2}$ & $6 \mathrm{~cm} \mathrm{~Pb}$ \\
\hline Target layer & $6.0 \mathrm{~m}^{2}$ & $70 \mathrm{~cm}$ Pitch & $44.2 \mathrm{~m}^{2}$ & $23 \mathrm{~cm}$ Pitch & $44.2 \mathrm{~m}^{2}$ & $30 \mathrm{~cm} \mathrm{CH}$ \\
\hline Air gap & -- & $80 \mathrm{~cm}$ & - & $158 \mathrm{~cm}$ & - & $237 \mathrm{~cm}$ \\
\hline Lower chamber & $6.0 \mathrm{~m}^{2}$ & $10 \mathrm{~cm} \mathrm{~Pb}$ & $32.0 \mathrm{~m}^{2}$ & $10 \mathrm{~cm} \mathrm{~Pb}$ & $32.0 \mathrm{~m}^{2}$ & $10 \mathrm{~cm} \mathrm{~Pb}$ \\
\hline
\end{tabular}

f Polyethylene

upon the chamber. It is worth noting,

(1) that hadron detection depends on the total thickness of lead in the chamber, because the mean free path of inelastic $N$-Pb collisions $(N$ : nucleon) is $\lambda_{N}=18.0 \mathrm{~cm}$, and

(2) that the observed energy $E_{o b}$ of hadroninduced shower is not that of the incident hadron $E_{h}$ but that released into $\gamma$-rays, i.e. $E_{o b}=k_{\gamma} E_{h}$ where $k_{\gamma}$ is the energy fraction of produced $\gamma$ rays with $\left\langle k_{\gamma}\right\rangle \simeq 1 / 6$. Therefore the detection threshold for hadrons is much higher ( $\sim 6$ times) than that for $(e, \gamma)$ 's.

\subsection{Nuclear interactions in $10^{14} \mathrm{eV}$}

Nuclear interactions in $10^{14} \mathrm{eV}$ are observed by the two-storey chamber (Fig. 2), which consists of the upper chamber, the target layer, the air gap and the lower chamber.[4,6] (Sce Table 2 for details of the two-storey chamber.) The upper chamber is a filter of atmospheric $(e, \gamma)$ 's, and therefore only the hadrons arrive at the target layer to cause multiple particle production. The $\gamma$-rays produced in multiple particle production, which are mainly the decay products of produced $\pi^{0}$ s, arrive at the lower chamber with sufficient mutual separation owing to the air gap. ${ }^{2}$ The events are called " $\mathrm{C}$-jets", because the events are mainly due to the collisions with carbon in the target layer.

The showers in the lower chamber, originated by these $\gamma$-rays, are inspected by a microscope in the nuclear emulsion plates which are employed in the sensitive layers of the lower chamber together with $\mathrm{X}$-ray films. Their energies are determined by counting the number of electron tracks in respective showers. That is, the transition of the electron number in a shower is compared with those which are calculated for various incident energies by cascade theory. [5] The threshold energy for shower detection is $E_{t h}=0.2 \sim 0.5 \mathrm{TeV}$ when we use nuclear emulsion plates.

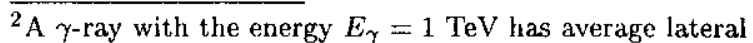
spread $r=I I p_{T_{\gamma}} / E_{\gamma}=100(\mathrm{~cm}) \times 200(\mathrm{MeV} / c) / 1(\mathrm{TeV})$ $=200(\mu \mathrm{m})$ after traversing the air gap of $H=1(\mathrm{~m})$.
} 

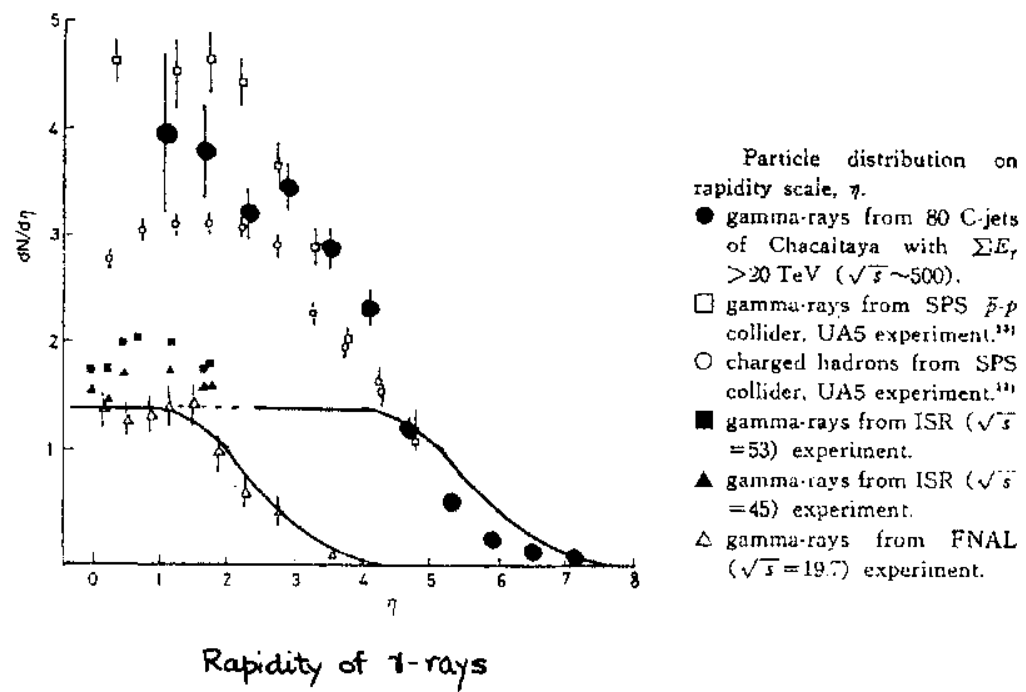

Figure 3. Rapidity density distributions of $\gamma$-rays. The solid curves present the distributions to follow Feynman scaling law at $s^{1 / 2}=19.7 \mathrm{GeV}$ and at $s^{1 / 2}=500 \mathrm{GeV}$, respectively.

The basic idea of two-storey chamber is to study nuclear collisions by observing $\pi^{0}$ 's among the produced particles in multiple particle production. One can see that the produced particles in the collision are observed directly in this experiment. It is worth noting that the ambiguity of the interaction point in the target layer is less than the error of energy, determined by electron track counting, and consequently the energy of $\gamma_{-}$ rays can be calibrated by the kinematical relation of $\pi^{0} \rightarrow 2 \gamma$ decay.

The characteristics of multiple particle production, which are obtained by the experiment, were confirmed by the experiments by CERN $\bar{p} p$ collider.[7] Those are the violation of Feynman scaling law, gradual increase of $\left\langle p_{T \gamma}\right\rangle$ (the average transverse momentum) with the incident energy, a correlation between $d N / d y_{\gamma}$ (the rapidity density of produced $\gamma$-rays) and $\left\langle p_{T_{\gamma}}\right\rangle$, etc.

Fig. 3 presents the rapidity density distribution of $\gamma$-rays obtained by the experiment. $[8,6]$ The data are $80 \mathrm{C}$-jets with the total observed energy $\sum E_{\gamma}=20 \sim 80 \mathrm{TeV}$ and the number of observed $\gamma$-rays $n_{\gamma} \geq 4$. The average incident energy of collision is $\langle\sqrt{s}\rangle=500 \mathrm{GeV}$. It shows clearly that $\gamma$-ray production is suppressed in the forward region and enhanced in the central region, compared with the distribution of the Feynman scaling law.

\subsection{Nuclear interactions in $10^{15} \mathrm{eV}$ (1)}

Direct observation of nuclear collisions is not easy in this energy region because of the scarce intensity of high energy cosmic-rays even at high mountains. Hence nuclear interactions in the atmosphere are observed by the upper chamber of the two-storey chamber or by a simple emulsion chamber of large area.

The event, called "family" or "A-jet", consists of several $\sim$ a few tens showers of $(e, \gamma)$ and hadron origins, distributed over an area of a few $\sim$ ten centimeters radius. Identification of family is easy because the constituent showers are in parallel one another on the projection map of all the observed showers. Family is the air shower at 


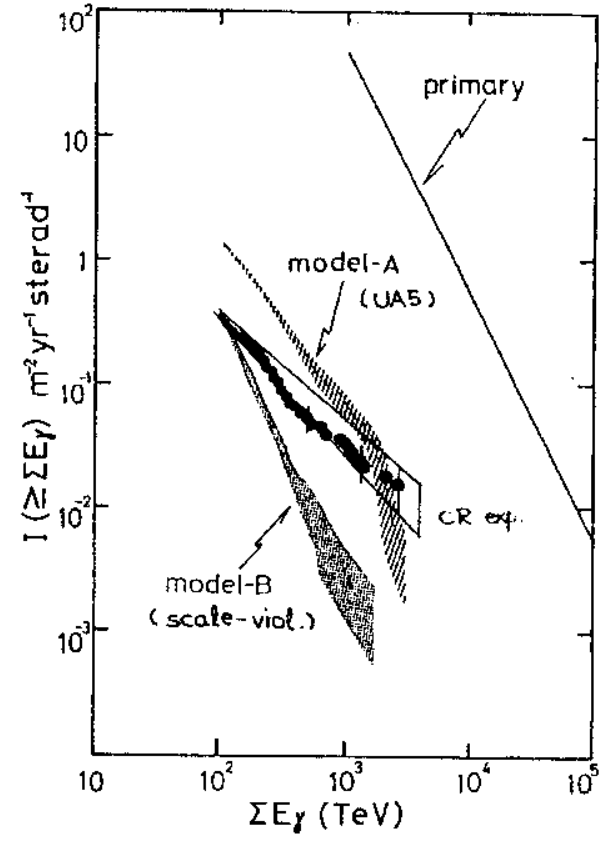

Figure 4. The intensity of families with the energy exceeding $\sum E_{\gamma}$ (the total observed energy in the family) at Pamirs. Full circles are the experimental data, and 'model-A' is by the simulations where UA5 code is assumed for nuclear interactions.

its central part or at the early stage of its development, because only the high energy particles are detected by emulsion chamber.

It is not straightforward to extract characteristics of nuclear collisions from the observed data of families, because most of the events are not due to a single collision but due to complicated cascade processes - nuclear and electromagnetic - in the atmosphere. Therefore usually the data are compared with simulations which follow the atmospheric diffusion of cosmic rays. Main assumptions in the simulations are on the energy distribution of produced particles in multiple particle production and on the mass composition of the primary cosmic rays. We assume UA5 code for the former and proton-dominant composition for the latter. UA5 code is a phenomenological simulation code of multiple particle production, which reproduces what UA5 Collaboration observed at CERN $\ddot{p} p$ collider.[9] It predicts the energy distribution of produced particles with rather strong violation of Feynman scaling law, both in the central region and in the forward region. (See Sect. III.) The assumed proton-dominant composition[10] is the extrapolation of the one in low energy region ( $\leq$ several $\mathrm{TeV}$ ), which are established by the experiments of direct observation. ${ }^{3}$

Fig. 4 shows the frequency of the families with the energy exceeding $\sum E_{\gamma}$ (the total observed energy of $(e, \gamma)$ 's in the family) at Pamir altitude by Chacaltaya-Pamir Collaboration,[11] which is one of the basic parameters to discuss the families. Those by simulations are presented together. The figure shows that the intensity by the experimental data is lower than that by the simulation where UA5 code and proton-dominant composition are assumed. It indicates that at least either of the assumptions should be revised in the direction to make energy subdivision more rapid through the atmospheric diffusion of cosmic rays. That is, the violation of Feynman scaling law is stronger than that expected by UA5 code and/or the composition of the primary cosmic rays is heavier than proton-dominant one in $10^{15} \mathrm{eV}$.

Fuji-Kambala collaboration obtained the similar results by the emulsion chamber experiment at Mt. Kambala $(5,400 \mathrm{~m}$, Tibet $)$, which are shown in Fig. 5.[12] They argue that the composition becomes quite heavier in $10^{15} \mathrm{eV}$ on the assumption of quasi-scaling energy distribution of the produced particles. However, a quasi-scaling energy distribution is not an established one in the cnergy region of $10^{15} \mathrm{eV}$.

\begin{tabular}{l|ccccc}
\hline${ }^{3}$ Typical one is; \\
$\begin{array}{c}E_{0}(\mathrm{eV}) \\
\end{array}$ & $\mathrm{H}$ & Relative abundance $(\%)$ & CNO & Heavy & Fe \\
\hline $10^{15}$ & 42 & 17 & 14 & 14 & 13 \\
$10^{16}$ & 42 & 13 & 14 & 15 & 16 \\
\hline
\end{tabular}



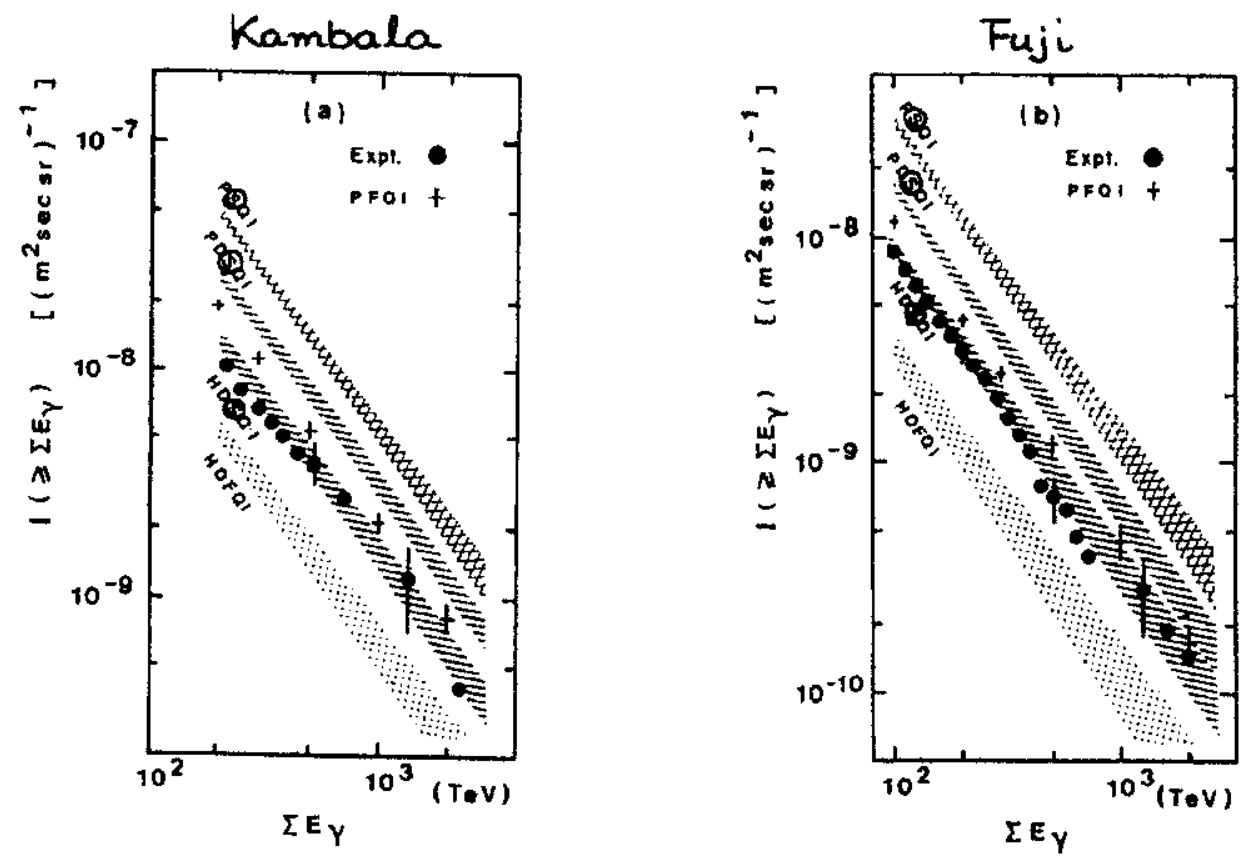

Figure 5. The intensity of the families with the energy excceding $\sum E_{\gamma}$ at Mt. Fuji and at Mt. Kambala. The abbreviations, attached to the data by simulations, are as follows.

$\mathrm{P}$ (PD) : protons (proton dominant composition) for primary cosmic rays

$\mathrm{S}(\mathrm{F})$ : scaling (fire-ball) model for nuclear interactions

$\mathrm{Q}$ : QCD-jets included

I : increasing cross section

2.4. Nuclear interactions in $10^{15} \mathrm{eV}(2)$

We cannot discriminate by emulsion chamber data which of nuclear interaction or cosmic-ray composition is the cause of the observed low intensity of families. Still more it should be pointed out that the above argument nceds further assumption of the intensity of the primary cosmic rays, which is obtained by the air shower experiments assuming characteristics of high energy nuclear interactions. ${ }^{4}$ To overcome such shortcom-

\footnotetext{
${ }^{4}$ It is true that the air shower size at the shower maximum does not depend strongly on the details of nuclear interactions and on the nature of the primary cosmic rays. Hence it is a good measure of the primary energy to initiate the air shower.
}

ings we started an experiment to observe families and air showers simultaneously.[13]

The experimental set-up consists of air shower array, hadron calorimeter and emulsion chamber, by which air shower size (the total number of charged particles in the air shower), hadron data and family data are obtained, respectively. The hadron data which are obtained by the hadron calorimeter, installed beneath the emulsion chamber, provide a clue to link air showers with families. That is, the families without arriving time and the hadron data with arriving time are correlated by the coincidence of their geometrical center, and the hadron data and the air showers by the coincidence of their arriving time. 

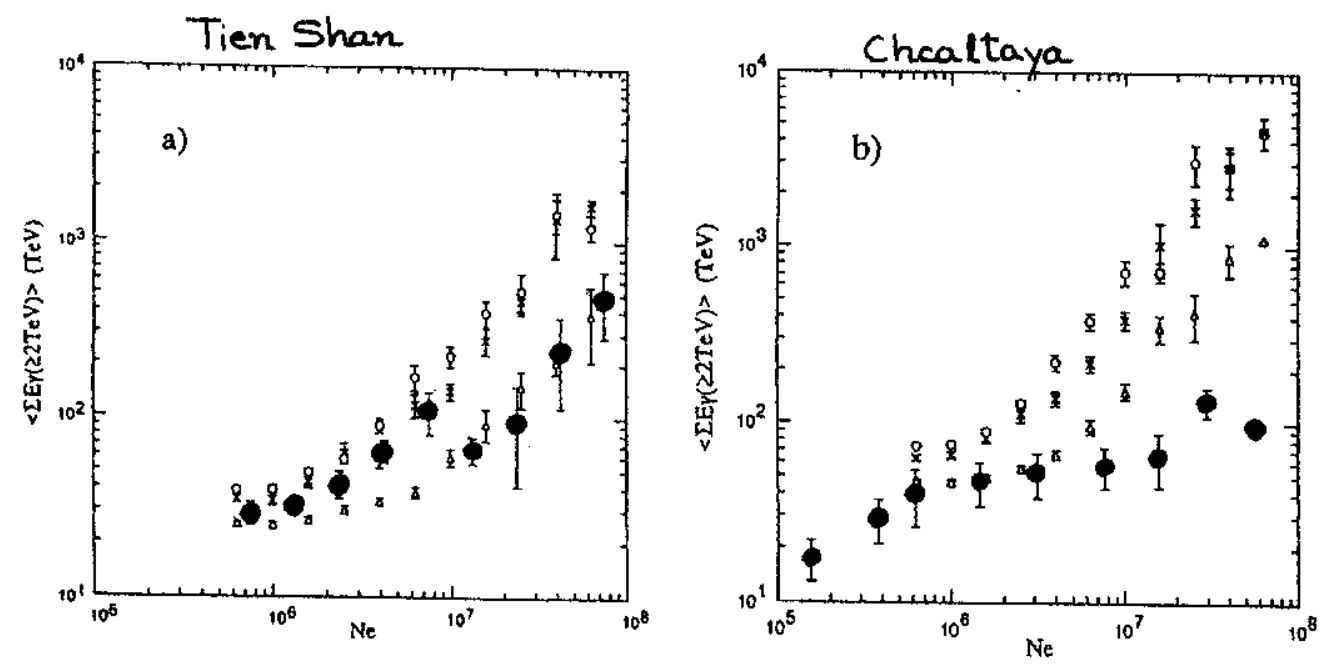

Figure 6. Shower-size $\left(N_{\mathrm{c}}\right)$ dependence of average family energy, $\left(\Sigma E_{\gamma}\right):(a)$ for the HADRON experiment at Tien-Shan, and (b) for the sys experiment at Chacaltaya. Symbols are $(O)$ for model-A with 'normal' chemical composition, $(X)$ model-A with 'heavy-dominant' chemical composition, and $(\Delta)$ model-B with 'normal' chemical composition and (S)experimentaldata.

Figure 6. Correlations between $\sum E_{\gamma}$ (total observed energy in the family) and $N_{e}$ (air shower size) at Tien Shan (a) and at Chacaltaya (b), for the air showers which are accompanied by families with $\sum E_{\gamma} \geq 10 \mathrm{TeV}$ and $n_{\gamma} \geq 4$. Full circles are for the experimental data open circles (crosses) are for those by simulations where UA5 code and proton-dominant (heavy-dominant) composition are assumed.

Fig. 6 presents a correlation between $\sum E_{\gamma}$ and $N_{e}$ (air shower size) for the air showers which are accompanied by the families. $[14,13]$ Definition of the family is $\sum E_{\gamma} \geq 10 \mathrm{TeV}$ and $n_{\gamma} \geq 4$. Discrepancy between the experimental data and the simulation leads to the same conclusion mentioned in the previous subsection. The similar type of the experiments, which is carried out at Tien Shan (3,300 m, Kazakhstan), obtained the same conclusion, which is shown in Fig. 6.[14, 15]

The figures show also that the hypothesis of the heavy composition of the primary cosmic rays, proposed by Fuji-Kambala Collaboration, reduces but cannot describe fully the observed discrepancy. Hence we conclude that violation of the Feynman scaling law is stronger than that assumed in the UA5 code, irrespective of the assumption of the primary cosmic-ray composition.

Fig. 7 shows the differential energy spectrum of hadrons in the air shower, which is obtained from the data by the hadron calorimeter, together with the results by the simulations.[16] The figure confirms the above conclusion again. KASCADE experiment reaches the same conclusion from the study of hadronic cores of extensive air showers by the large hadron calorimeter.[17]

\section{Nuclear Interactions at $10^{14} \sim 10^{20} \mathrm{eV}$}

As we saw in the previous sections, emulsion chamber experiments show/indicate that the Feynman scaling law of the energy distribution of produced particles, which looks to be valid in $\sqrt{s} \leq 63 \mathrm{GeV}$, is violated in $10^{14} \sim 10^{16} \mathrm{eV}$. Then our next question is what kind of energy distribution describes the experimental data. However there are a variety of energy distributions, possible physically. 


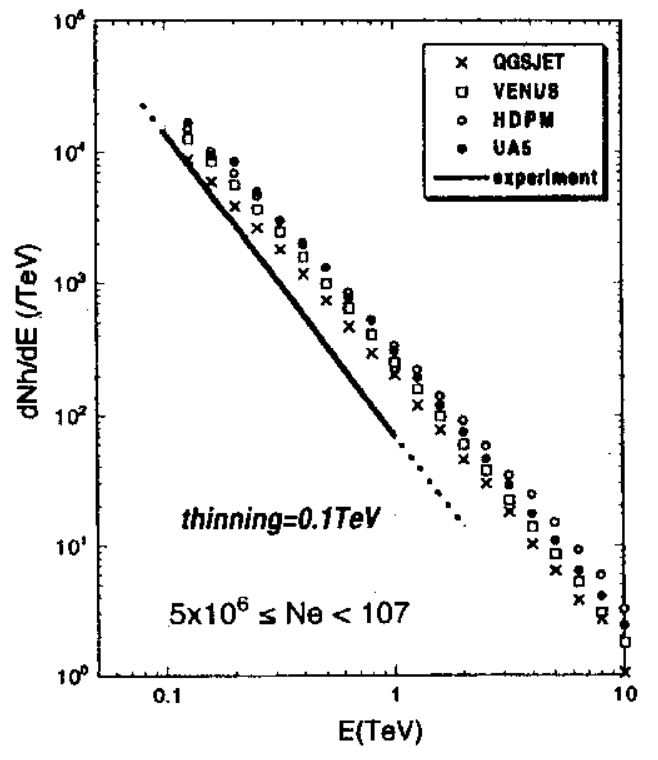

Figure 7. Differential number of hadrons in the air shower, which is obtained by the hadron calorimeter. Full circles are for the data by the simulation where UA5 code and proton-dominant composition are assumed.

Hence in this section we formulate the energy distribution of produced particles, based on the data of direct observation by accelerator experiments and cosmic-ray experiments (C-jets), which show violation of the law. Then the consequences of the formulated distribution are examined and the distribution is compared with those by nuclear interaction models which are used recently in simulations to follow the diffusion of cosmic rays in the atmosphere.

\subsection{Formulation of the energy distribution by the data of direct observation}

There are several sets of data of direct observation by cosmic-ray and accelerator experiments, which are tabulated in Table 3 in chronological order. One should notice that the experimental data are presented in various quantities as rapidity density, pseudo-rapidity density, etc., owing to the experimental conditions of the respective groups. To discuss these data in relation to the energy distribution of eq.(1), one has to transform $d N / d x^{*}$ into $d N / d \eta^{*}, d N / d y^{*}$, etc.

We assume that $x$-distribution is expressed in the following way.

$$
\begin{gathered}
\frac{d N}{d x^{*} d p_{T}}=a D \frac{\left(1-a^{\prime} x^{*}\right)^{d}}{\sqrt{x^{* 2}+\left(\frac{2 \mu}{\sqrt{s}}\right)^{2}}} g\left(p_{T}\right) \\
\left(\mu \equiv \sqrt{p_{T}^{2}+m_{\pi}^{2}}\right)
\end{gathered}
$$

where the parameters $a$ and $a^{\prime}$ are adjustable ones. This formula reproduces the scaling function of eq.(1) using $a=a^{\prime}=1$ and $\sqrt{s} \rightarrow \infty$. The parameters $a(\geq 1)$ and $a^{\prime}(\geq 1)$ express enhancement of eq.(1) in the central region and suppression in the forward region, respectively.

The $p_{r}$-distribution is assumed to be

$g\left(p_{T}\right) d p_{T}=p_{T} \exp \left(-\frac{p_{T}}{p_{0}}\right) \frac{d p_{T}}{p_{0}^{2}}$

with

$p_{0}=\left\{\begin{array}{ll}c & \left(x^{*}<x_{0}^{*}\right) \\ c\left(\frac{x_{0}^{*}}{x^{*}}\right)^{c^{t}} & \left(x^{*}>x_{0}^{*}\right)\end{array}(c=0.2 \mathrm{GeV} / c)\right.$

According to eq.(3), the average value of $p_{T}$, given by $\left\langle p_{T}\right\rangle=2 p_{0}$, is constant $(=0.4 \mathrm{GeV} / c)$ in $0 \leq x^{*} \leq x_{0}^{*}$ and becomes smaller in the forward region of $x^{*}>x_{0}^{*}$, which is observed by the experiments. $[4,18]$ The values of $c^{\prime}$ and $x_{0}^{*}$ are determined as

$c^{\prime}=0.57, \quad x_{0}^{*}=0.08$

by the rapidity dependence of the average $p_{T}$ value by UA7 Collaboration.[18]

The energy distribution of eq.(2) can be transformed easily to rapidity and pseudo-rapidity distributions;

$$
\begin{aligned}
& \frac{d N}{d y^{*} d p_{T}}=a D\left[1-a^{\prime} \frac{\mu}{\sqrt{s}}\left(e^{y^{*}}-e^{-y^{*}}\right)\right]^{d} g\left(p_{T}\right) \\
& \frac{d N}{d \eta^{*} d p_{T}}=a D\left[1-a^{\prime} \frac{p_{T}}{\sqrt{s}}\left(e^{\eta^{*}}-e^{-\eta^{*}}\right)\right]^{d}
\end{aligned}
$$


Table 3

Particle distribution in multiple particle production

\begin{tabular}{|c|c|c|c|c|c|c|c|}
\hline Group & Site & Collision & $\begin{array}{c}\text { Energy } \\
\sqrt{s}(\mathrm{GeV})\end{array}$ & $\begin{array}{l}\text { Maximum } \\
\text { rapidity }\end{array}$ & $\begin{array}{l}\text { Presented } \\
\text { quantity }\end{array}$ & $\begin{array}{l}\text { Observed } \\
\text { particles }\end{array}$ & $\begin{array}{l}\text { Observed } \\
\text { region }\end{array}$ \\
\hline $\begin{array}{l}\text { Brazil-Japan } \\
\text { Collab.[6] }\end{array}$ & $\begin{array}{l}\text { Mt. Cha- } \\
\text { caltaya }\end{array}$ & $h-\mathrm{C}^{\dagger}$ & $500 \ddagger$ & 12.2 & $d N / d y_{\gamma}$ & $\gamma$-rays & $y_{\gamma} \geq 7.8$ \\
\hline UA5 Collab.\{9] & CERN & $\grave{p} p$ & $\begin{array}{r}53 \\
200 \\
546 \\
900\end{array}$ & $\begin{array}{l}4.0 \\
5.3 \\
6.3 \\
6.8\end{array}$ & $\begin{array}{l}d N / d \eta^{*} \\
d N / d \eta^{*} \\
d N / d \eta^{*} \\
d N / d \eta^{*}\end{array}$ & $\begin{array}{l}\text { charged } \\
\text { charged } \\
\text { charged } \\
\text { charged }\end{array}$ & $\left|\eta^{*}\right|<4.5$ \\
\hline UA7 Collab.[18] & CERN & $\bar{p} p$ & 630 & 6.4 & $d N / d y^{*}$ & $\pi^{0}$ & $y^{*}=5.0 \sim 6.6$ \\
\hline Harr et al.[19] & CERN & $\bar{p} p$ & 630 & 6.4 & $d N / d \eta^{*}$ & charged & $\eta^{*}=1.5 \sim 5.5$ \\
\hline \multicolumn{8}{|c|}{ hadron (nucleon, pion)-Carbon collisions } \\
\hline$\times \frac{\left(e^{\eta^{\prime}}\right.}{\sqrt{\left(e^{\eta^{*}}-t\right.}}$ & $\frac{\left.+e^{-\eta^{*}}\right)}{\left.=-\eta^{*}\right)^{2}+(i}$ & $g\left(p_{T}\right)$ & (5) & & $=1.67 \times 0$. & $\times\left(\frac{s}{s_{0}}\right)^{\alpha}$ & \\
\hline
\end{tabular}

The distributions $d N / d y^{*}$ and $d N / d \eta^{*}$ are obtained by numerical integration with respect to the transverse momentum $p_{T}$.

\subsection{Scaling violation parameters}

Fig. 8 shows the pseudo-rapidity distributions of eq.(5) at $\sqrt{s}=546 \mathrm{GeV}$ for various values of the scaling violation parameter $a^{\prime}$. We assume the energy dependence of parameter $a$ as

$a=\left(\frac{s}{s_{0}}\right)^{\alpha} \simeq\left(\frac{E_{0}}{A}\right)^{\alpha}$

where $s_{0}=3.9 \times 10^{2} \mathrm{GeV}^{2}, A \equiv s_{0} / 2 M=$ $2.0 \times 10^{2} \mathrm{GeV}$ and $\alpha=0.105$. Then the pseudorapidity density at $\eta^{*}=0$ is given by

$$
\left(\frac{d N}{d \eta^{*}}\right)_{\eta^{*}=0}=D\left\langle\frac{p_{r}}{\sqrt{p_{T}^{2}+m_{\pi}^{2}}}\right\rangle a
$$

which reproduces $\rho(0)=0.74 s^{0.105}$, given empirically by UA5 Collaboration.[9] It may be worth mentioning that the data of $\rho(0)$, the pseudorapidity density at $\eta^{*}=0$, is reliable by a collidertype accelerator experiment.

The calculated distributions are compared with the experimental data, listed in Table 3 , to obtain the value of the scaling violation parameter $a^{\prime}$. The data are those of all inelastic events, but not of NSD (non-single-diffractive) events, because the former is more suitable for discussing cosmic-ray diffusion. ${ }^{5}$ The energy dependence of

\footnotetext{
${ }^{5}$ Definitions of all inelastic events and NSD events are, $\sigma_{\text {inel }}=\sigma_{\mathrm{NSD}}+\sigma_{\mathrm{SD}} \quad$ and $\quad \sigma_{\mathrm{NSD}}=\sigma_{\mathrm{ND}}+\sigma_{\mathrm{DD}}$ where ND, SD and DD stand for "non-diffractive", "singlediffractive" and "double-diffractive" events.

According to the data by UA5 Collaboration, the density of the former is smaller by $10 \%$ than that of the latter in the pseudo-rapidity range $0<\eta^{*} \leq 3.5$ and is almost equal to that of the latter in $\eta^{*} \geq 3.5$ at $\sqrt{s}=546 \mathrm{GeV}$.[9]
} 


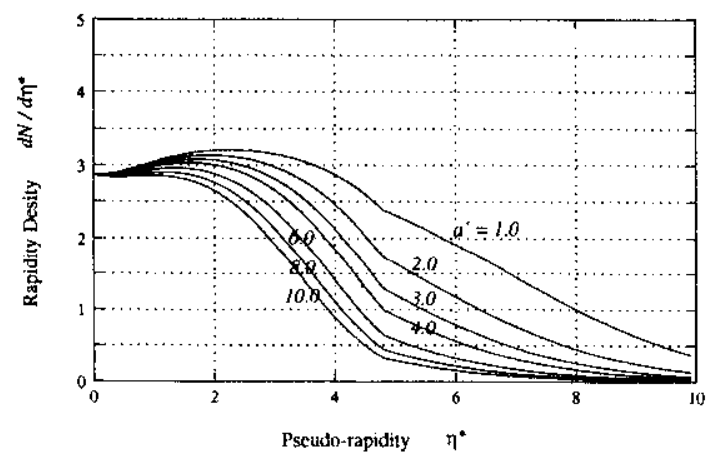

Figure 8. Pseudo-rapidity density distributions at $s^{1 / 2}=546 \mathrm{GeV}$ for various values of the scaling violation parameter $a^{\prime}$. The parameter $a$ is assumed by eq.(6) in the text. The kink of the curve at $\eta^{*}=4.8$ is due to the rapidity dependence of the average $p_{T}$ value.

the scaling violation parameters $a$ and $a^{\prime}$ are in Fig. 9. There we observe that the parameter $a^{\prime}$ has a stronger dependence than the parameter $a$.

Two cases of energy dependence are used in

$a^{\prime}=\left(\frac{E_{0}}{A}\right)^{\alpha^{\prime}} \quad\left(\alpha^{\prime}=0.105\right.$ and 0.210$)$

which are called Model-1 and Model- 2 hereafter, respectively. The parameters $a$ and $a^{\prime}$ in Model-1 have the same energy dependence, and those in Model-2 are the best-fitting ones to the experimental data. The values of $a^{\prime}$ by Harr et al. and by $\mathrm{C}$-jets deviate considerably from other data. Model- 0 with $a=a^{\prime}=1.0$, which stands for the case of Feynman scaling law, is for the reference. (Table 4.)

Fig. 10 shows the pseudo-rapidity density distributions (of all inelastic events but not of nonsingle-diffractive events) by UA5 Collaboration together with those of the parameter values of the best fitting. One can see in the figure that the reproduction is satisfactory by Model- 2 and that Model-0 (the Feynman scaling law) cannot reproduce the data both in the central and forward regions.

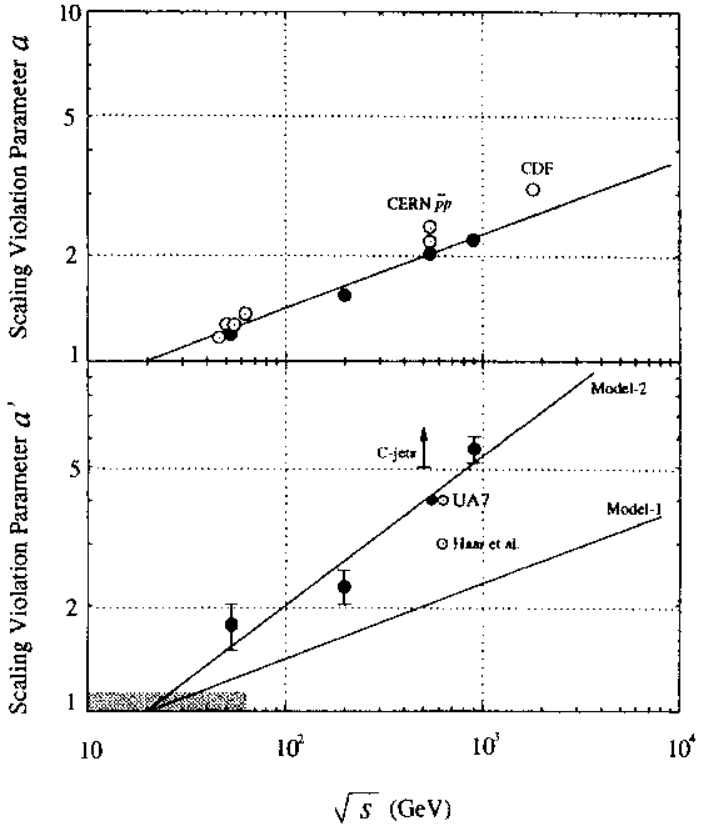

Figure 9. Energy dependence of the scaling violation parameters, $a$ and $a^{\prime}$, which are obtained by fitting the calculated curves of (pseudo-)rapidity density distribution to those of the experimental data in Table 3 . The full circles are the experimental data of UA5 Collaborationand the open circles are by the data of other experimental groups. The hatched area indicates the energy region where the Feynman scaling law ( $a \simeq 1.0$ and $a^{\prime} \simeq 1.0$ ) is verified by the experiments. The lines are the assumed energy dependences of Model-1 and Model-2. 
Table 4

Scaling violation parameters, assumed in the models

$\begin{array}{llll}\text { Model } & \text { Scaling-violation parameters* } & \text { Average } & \text { Remarks } \\ & a=\left(\frac{E_{0}}{A}\right)^{\alpha} \quad a^{\prime}=\left(\frac{E_{0}}{A}\right)^{\alpha^{\prime}} & \text { inelasticity } & \end{array}$

Model-0 $\quad \alpha=0 \quad \alpha^{\prime}=0 \quad<K>=0.5 \quad$ the Feynman scaling law

Model-1 $\quad \alpha=0.105 \quad \alpha^{\prime}=0.105 \quad<K>=0.5$

Model-2 $\alpha=0.105 \quad \alpha^{\prime}=0.210 \quad<K>=\left(\frac{E_{0}}{A}\right)^{-0.105} \begin{aligned} & \text { consistent with experimental } \\ & \text { data }\end{aligned}$

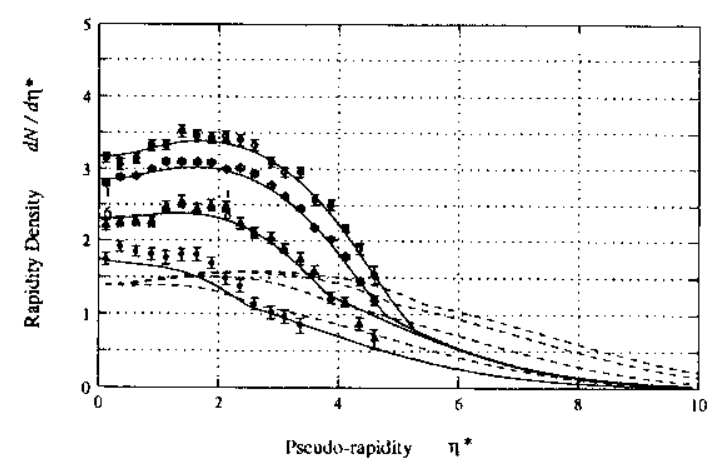

Figure 10. Pseudo-rapidity density distributions. Experimental data are by UA5 Collaboration for all inelastic events at the energies of $s^{1 / 2}=53$ $\mathrm{GeV}(\bullet), 200 \mathrm{GeV}(\triangle), 546 \mathrm{GeV}(\diamond)$, and $900 \mathrm{GeV}$ (o). Solid lines are the eq. $(5)$ in the text, with the parameter values of the best fitting, shown in Fig. 2. The chain lines are those of Model- 0 .

\section{3. $x$-distribution, multiplicity and inelas- ticity, predicted by the models}

Using an approximate relation $x^{*} \simeq E / E_{0} \equiv$ $x$, valid at high energies, we obtain $d N / d x$ from $d N / d x^{*}$ of eq.(2).

$$
\frac{d N}{d x}=\int_{0}^{\infty} a D \frac{\left(1-a^{\prime} x\right)^{d}}{\sqrt{x^{2}+\left(\frac{2 \mu}{\sqrt{s}}\right)^{2}}} g\left(p_{T}\right) d p_{T}
$$

Fig. 11 shows the $x$-distributions, predicted by Model-2. As can be seen in the figures, the Feynman scaling law is violated strongly both in the central region and in the forward region in Model2. There is almost no particle with $x \geq 0.01$ at $E_{0}=10^{20} \mathrm{eV}$.

Fig. 12 shows the energy dependence of charged multiplicity, predicted by the models, after integration of eq.(7). That is,

$m\left(E_{0}\right)=\int_{0}^{1 / a^{\prime}} d x \frac{d N}{d x}$

One can see in the figure that there is no big difference about the multiplicity between Model1 and Model-2. And it is of no wonder that the energy dependence of Model-2 agrees better with the experimental data than that of Model-1 does.

Fig.12 shows also the energy dependence of the average total inelasticity in the laboratory system, defined by

$$
<K>=\frac{3}{2} \int_{0}^{1 / a^{\prime}} x \frac{d N}{d x} d x
$$

It shows that the inelasticity decreases considerably in Model-2 at high energies while it is con- 

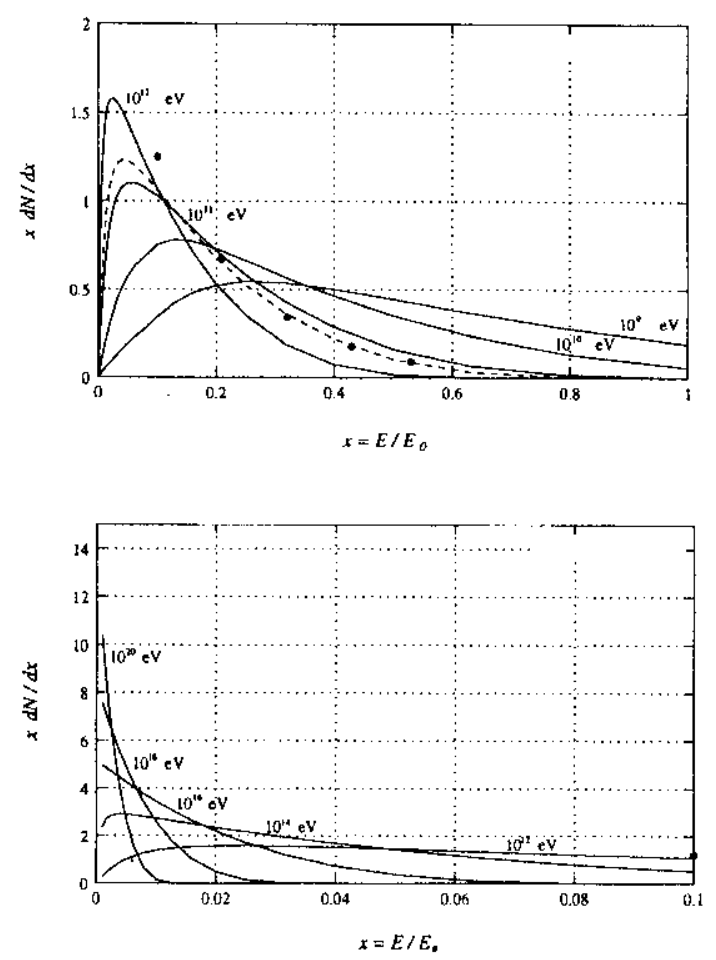

Figure 11. $x$-distribution of Model-2 for various primary energies $E_{0}$. The model describes the strong violation of Feynman scaling law both in the forward region and in the central region. The chain line in the upper figure is the scaling function of eq.(1) in the text. Full circles are the experimental data of $p+p \rightarrow \pi^{c h}+X$ at $s^{1 / 2}=20$ $\mathrm{GeV}$, where the scaling law is valid.[20] The lower figure shows that the distribution in the forward region shrinks distinctly, in particular, at highest energies.

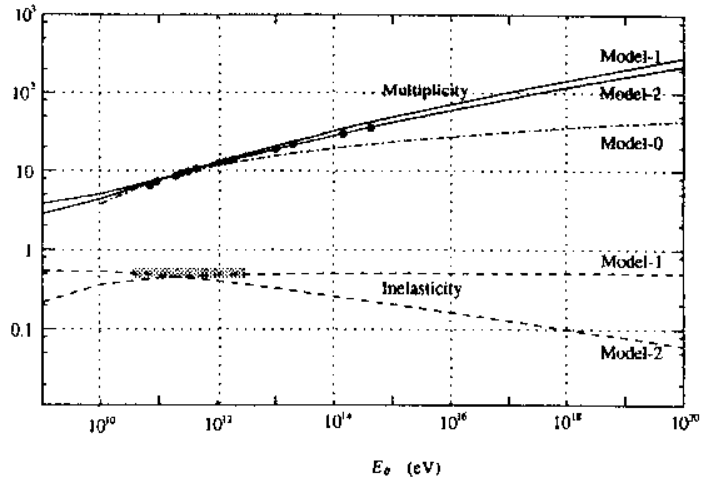

Figure 12. Energy dependence of charged multiplicity and that of total inelasticity, predicted by the models. There is no big difference of the multiplicity between the models. One can see that Model-2 describes the experimental data (full circles) better than Model-1 does, because Model2 has the best fitted parameters to describe the rapidity density distribution. Inelasticity is decreasing in Model-2, while it is constant $(=0.5)$ for Model- 0 and Model-1. The shadowed area indicates the region where the Feynman scaling law is verified by the experiments within the experimental errors. 
stant $(=0.5)$ in Model-0 and in Model-1. ${ }^{6}$

\subsection{Comparison with models used in sim- ulations}

It is interesting to sec how the relation is between the models, assumed here, and those which are used recently in simulations of atmospheric cosmic-ray diffusion. We compared in Fig. 13 the pseudo-rapidity density distributions[21], predicted by UA5 code[22] ${ }^{7}$, VENUS[23], QGSJET[24], SIBYLL[25], HDPM[26] and DPMJET[27], with those of the present models. ${ }^{8}$ The models VENUS, QGSJET and DPMJET are QCD theory inspired ones, while UA5 code, SIBYLL and HDPM are based on the experimental data.

One can see the following points in the figure. (1) The difference among model predictions is not small one another, although all models are either QCD-inspired or empirical.

(2) In the central region all model predictions of the pseudo-rapidity density are consistent, except that of HDPM.

(3) In the middle pseudo-rapidity region, i.e. $2.0 \leq \eta^{*} \leq 6.0$, which is the most important for the atmospheric diffusion of cosmic rays, QCDinspired models predict higher density than phenomenological models do. The pseudo-rapidity densities by the QCD-inspired models, are between those of Model-1 and Model-2. Those by the phenomenological models are almost consistent with Model-2.

(4) In the most forward region, all model predictions are consistent with the one of Model-2.

\footnotetext{
${ }^{6}$ It may look strange that the average inelasticity $\langle K\rangle$ is 0.5 for Model-1, which has a higher rapidity-density than QGSJET, because it is said that $\langle K\rangle \simeq 0.6$ by QGSJET. It is due to the difference of sampled events, i.e. ail inelastic events in the former and NSD events in the latter. In other words the average inelasticity by QGSJET is $\sim 0.5$ for all inelastic events.

${ }^{7} \mathrm{UA5}$ code is a phenomenological simulation program, made by UA5 Collaboration, which describes the data observed by the collaboration. The code does not necessarily predict the pseudo-rapidity density correctly in the forward region, because the observed pseudo-rapidity region by UA5 Collaboration is limited to $\eta^{*} \leq 4.5$.

${ }^{8}$ One should notice that the pseudo-rapidity density by simulations is for NSD (non-single-diffractive) events while that of the calculation is for all inelastic events. See the footnote ${ }^{6}$
}

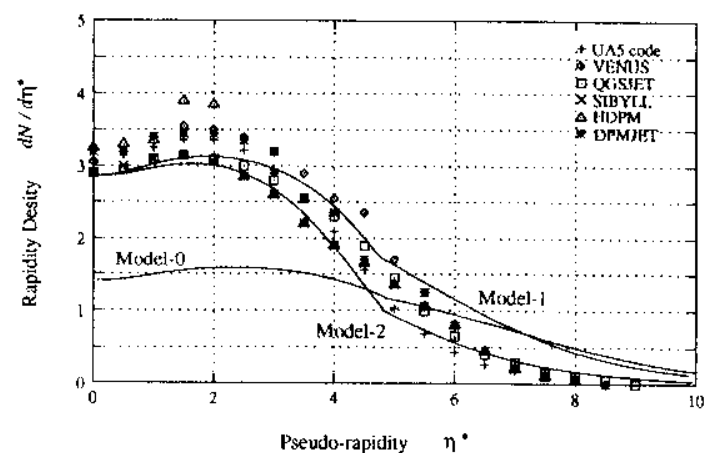

Figure 13. Pseudo-rapidity density distributions at $s^{1 / 2}=546 \mathrm{GeV}$. Plots are by the models (UA5 code, VENUS, QGSJET, SIBYLL, HDPM and DPMJET) which are employed in the simulations of cosmic ray phenomena. The data by the simulations are based on the NSD (nonsingle-diffractive) events, while those by Model-0, Model-1 and Model-2 (solid lines) are for all inclastic events. See the footnote ${ }^{6}$ for the difference between NSD and all inelastic events.

(5) The experimental data by Harr et al. are almost consistent with those by QGSJET.

(6) The distribution by UA5 code is almost consistent with that of Model-2.

\section{Application of the formulated energy distribution to highest energy air show- ers}

We consider highest energy air showers $\left(>10^{18}\right.$ $\mathrm{eV}$ ) as the first step to examine the validity of the formulated energy distribution of produced particles, because the energy dependence of the formulated distribution has larger effect at higher energies.

Our interest is how the nuclear interactions affect the air shower development and how the formulated energy distribution, based on the data of direct observation, describes the highest energy air showers. 


\subsection{Diffusion of Cosmic Rays in the At- mosphere.}

A high energy primary cosmic-ray proton ${ }^{9}$, incident upon the top of the atmosphere, makes a nuclear collision with an atmospheric nucleus, and many particles -- one surviving particle and a number of produced particles --. are yielded through the collision. The inelastic cross section of the proton-air collision increases with the proton energy. The surviving particle, either a proton or a neutron, repeats inelastic collisions in the atmosphere.

The produced particles are assumed to be pi ons. The energy distribution of produced pions is substituted by that of $N-N$ collision, which is formulated in the previous paper, because the effect of the air nucleus target appears only in the backward region. ${ }^{10}$ The charged pions among the produced pions make nuclear collisions in the atmosphere again. The collision mean free path of charged pions has the same energy dependence as that of nucleon. We neglect decays of charged pions into muons.

The multiple particle production, induced by a charged pion, has essentially the same characteristics as the onc by a nucleon, which is confirmed in low energy region within the errors of the experimental data. That is, the final state of the collision consists of one surviving pion and produced pions whose energy distribution is the same as the one of proton collision. The differences are that the inelastic cross section of proton is larger than that of pion, and that the surviving pion has a probability to be a neutral pion (callcd the 'charge exchange' process). (See Table 5.)

The neutral pions, annong the produced pions and through the charge exchange process, decay into $\gamma$-rays which produce a number of electromagnetic component, electrons and photons, via electromagnetic cascade process.

\footnotetext{
${ }^{9}$ We assume that the primary cosmic ray is a proton. And we employ the superposition model for a target nucleus, which assumes that the nucleus of mass number $A$ and energy $E_{0}$ is a bundle of $A$ nucleons and energy $E_{0} / A$, each. The model is valid when the observation level is sufficiently deep in the atmosphere.[?]

${ }^{10}$ The diffusion of cosmic rays in the atmosphere is governed by the high energy particles, produced in the forward region.
}

Following the above description of the cosmicray diffusion in the atmosphere, we formulate the elementary processes in the following way.

(1) Inelastic collision mean free path

The mean free path of the inelastic $N-\operatorname{air}(N$ : nucleon) collisions,

$\lambda_{N}\left(E_{0}\right)=\lambda_{N}\left(\frac{E_{0}}{B}\right)^{-\beta} \quad\left(\lambda_{N}=80.0 \mathrm{~g} / \mathrm{cm}^{2}\right)$

and for $\pi^{ \pm}-$air collisions

$\lambda_{\pi}\left(E_{0}\right)=\frac{1}{\xi} \lambda_{N}(E) \quad\left(\xi \equiv \frac{\sigma_{\pi N}}{\sigma_{N N}}=0.71\right)$

(2) Energy distribution of produced charged pions

The energy distribution of produced charged particles through multiple particle production is formulated in Section 3. In the laboratory system it is given by eq.(7). And the scaling violation parameters $a$ and $a^{\prime}$ are dependent on the incident energy. That is,

$a=\left(\frac{E_{0}}{A}\right)^{\alpha} \quad a^{\prime}=\left(\frac{E_{0}}{A}\right)^{\alpha^{\prime}}$

where the exponents of the parameters $\alpha$ and $\alpha^{\prime}$ are tabulated in Table 4.

In other words we assume that the number of charged pions with the energy between $E$ and $E+$ $d E$ is given by

$\varphi\left(E_{0}, E\right) d E=D\left(\frac{E_{0}}{A}\right)^{\alpha}\left[1-\left(\frac{E_{0}}{A}\right)^{\alpha^{\prime}} \frac{E}{E_{0}}\right]^{d} \frac{d E}{E}$

where $E_{0}$ is the energy of the incident particle. According to the above, the energy of the produced pions is between $E=0$ and $E=$ $\left(E_{0} / A\right)^{-\alpha^{\prime}} E_{0}$.

The average value of the total inelasticity in the laboratory system it is constant $(=0.5)$ in Model0 and Model-1, while it decreases considerably in Model-2 at high encrgies. (See Fig. 12 and Table 4.)

(3) Inelasticity distribution

We assume that the inelasticity is distributed uniformly between 0 and $2\langle K\rangle(\leq 1.0)$. That is, the inelasticity distribution is

$u(K) d K=\frac{d K}{2<K>} \quad(K=0 \sim 2<K>)$ 
Table 5

Energy distribution of the produced particles in multiple particle production

Collision Surviving particle Produced particles (pions)

\begin{tabular}{ccc}
\hline \multirow{N}{*}{$N-N$} & nucleon & charged pions \\
& $\delta\left(E-(1-K) E_{0}\right) d E$ & $\varphi\left(E_{0}, E\right) d E$ \\
& & neutral pions \\
& & $\frac{1}{2} \varphi\left(E_{0}, E\right) d E$ \\
& & \\
& charged pions & charged pions \\
$\pi^{ \pm}-N$ & $(1-b) \delta\left(E-(1-K) E_{0}\right) d E^{\dagger}$ & $\varphi\left(E_{0}, E\right) d E$ \\
& neutral pions & neutral pions \\
& $b \delta\left(E-(1-K) E_{0}\right) d E^{\dagger}$ & $\frac{1}{2} \varphi\left(E_{0}, E\right) d E$ \\
& & \\
\hline
\end{tabular}

$\dagger$ The charge exchange probability $b=0.3$ is assumed.

(4) Energy spectrum of the surviving particle

The energy distribution of the surviving particle is determined by the inelasticity distribution;

$\int u(K) d K \delta\left(E-(1-K) E_{0}\right) d E$

which leads to $x$-distribution of the surviving particle as

$\frac{d x}{2<K>} \quad(1-2<K>\leq x \leq 1)$

That is, the variable $x$ of the surviving particle is distributed uniformly between $1-2\langle K\rangle$ and 1 , which is consistent with experimental data in low energy region.

There is a probability in pion collisions that the surviving pion is a neutral pion $\pi^{0}$, which decays immediately into $\gamma$-rays. This charge exchange process is important from the energy flow point of view, because the surviving particle has high energy in average, compared with the produced pions. We assume that the charge exchange probability is $b=0.3$ tentatively, because there is no experimental data of it in high energy region. ${ }^{11}$

(5) Energy distribution of $\pi^{0}$ 's

11The probability may be higher than 0.3 , and probably $\sim 0.4$.
We assume that the energy distribution of $\pi^{0} \mathrm{~s}$ is a half of that of charged pions, which is confirmed within the experimental errors in low energy region.

The assumptions, mentioned here, are tabulated in Table 5.

\subsection{Size of the air shower}

Size of the air shower is defined as the total number of charged particles in the air shower which pass through the plane at the obscrvation level. And the charged particles in an air shower consists of electron component (electrons and positrons), charged hadrons (mainly charged pions) and muons, among which electron component are dominant. Hence we calculate only the number of electron component $N_{e}$ to express the air shower size.

The calculation is made analytically, because our main interest is to see how the characteristics of nuclear interactions affect the air shower development. That is, the diffusion equations of nucleon, charged pion and electron components are solved analytically for four cases in Table 6 . It corresponds to take into account the respective factors of the nuclear interaction characteristics one by one, taking Case A as the zero-th approximation, which is the one of the objectives of the 
Table 6

Cases to solve the diffusion equations

\begin{tabular}{ccccccccc}
\hline Case & \multicolumn{4}{c}{ Parameters } & \multicolumn{3}{c}{ Remarks } \\
& $\alpha$ & $\alpha^{\prime}$ & $\beta$ & $b$ & Model & $\langle\bar{K}\rangle$ & $\sigma(E)$ \\
\hline $\mathrm{A}$ & 0 & 0 & 0 & 0 and 0.3 & Model-0 & $\rightarrow$ & $\rightarrow$ \\
$\mathrm{B}$ & 0 & 0 & 0.056 & 0 & Model-0 & $\rightarrow$ & $\nearrow$ \\
$\mathrm{C}$ & 0.105 & 0.105 & 0 & 0 & Model-1 & $\rightarrow$ & $\rightarrow$ \\
$\mathrm{D}$ & 0.105 & 0.210 & 0 & 0 & Model-2 & $\searrow$ & $\rightarrow$ \\
\hline
\end{tabular}

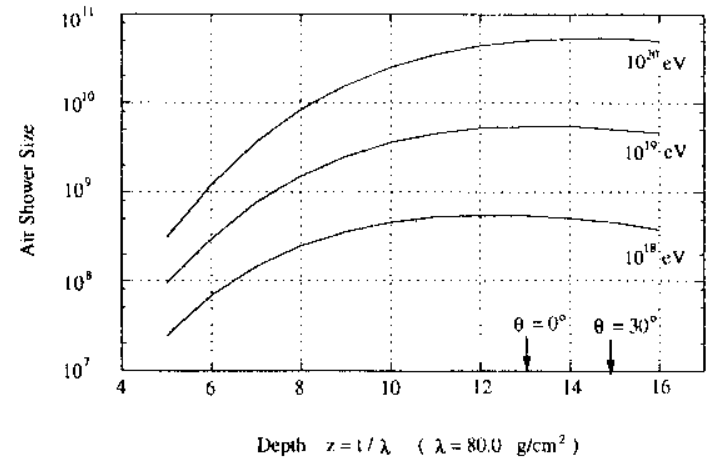

Figure 14. Transition curve of the air shower size for the primary proton with the energy $E_{0}=$ $10^{18}, 10^{19}$, and $10^{20} \mathrm{eV}$, for Case A (Model-0 and constant cross section). The arrows indicate the depth of the sea level $\left(1,030 \mathrm{~g} / \mathrm{cm}^{2}\right)$ for the air showers with the inclination $\theta=0^{\circ}$ and $30^{\circ}$.

present study.

Fig. 14 shows the transition curve of the air shower size for the primary proton with the energies $E_{0}=10^{18}, 10^{19}, 10^{20} \mathrm{eV}$ for Case A. One can see in the figure that the air showers are at the maximum development at sea level and that the relation $E_{0} / N_{e} \simeq 2.0(\mathrm{GeV})$ holds approximately.

Fig. 15 shows the ratio of the air shower size between the cases of $\mathrm{B}, \mathrm{C}$ and $\mathrm{D}$ and the case $\mathrm{A}$ for the primary energy $E_{0}=10^{20} \mathrm{eV}$. One can see the following in the figure.

(1) Effect of the charge exchange process of the surviving pion is almost constant over the atmospheric depth, and amounts $13 \%$. (This tendency can be explained by analytic solutions obtained.)

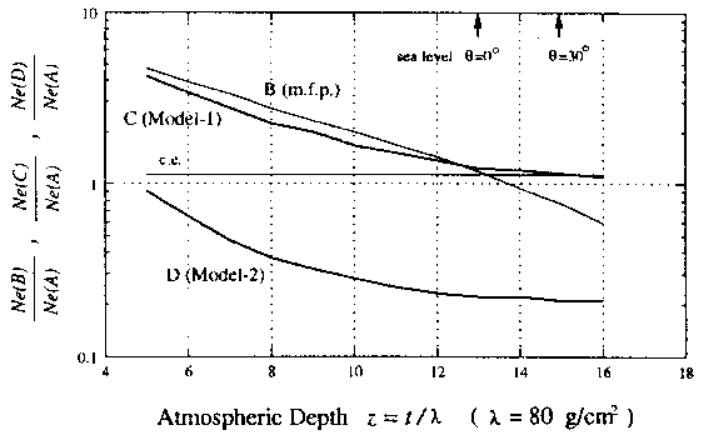

Figure 15. Ratio of air shower size, $N_{e}(\mathrm{~B}) / N_{e}(\mathrm{~A})$, $N_{e}(\mathrm{C}) / N_{e}(\mathrm{~A})$ and $N_{e}(\mathrm{D}) / N_{e}(\mathrm{~A})$, along the depth. The cases of $A, B, C$ and $D$ are tabulated in Table 6 . The primary energy of a proton is $10^{20} \mathrm{eV}$.

(2) Effect of increasing cross section is large at high altitude, but is small at sea level. (This tendency can be explained by analytic solutions obtained.)

(3) The effects of scaling violation, in Model-1 and in Model-2, have similar depth dependence, but the absolute values of them differ by five times. (4) Model-2 gives smaller air shower size, and the attenuation of the air shower size after the shower maximum is very slow due to the small value of inelasticity.

(5) At sea level the air shower size is affected most by the energy distribution of produced particles, and the effects of the increasing cross section and of the charge exchange are relatively small. 
Table 7

Constants in the numerical calculation

$$
\begin{aligned}
& \text { charge exchange probability } \quad b=0.3 \\
& \text { (of the surviving pion) } \\
& \text { collision mean free path } \\
& \begin{aligned}
N-\text { air } & \lambda_{N}=80.0\left(\mathrm{~g} / \mathrm{cm}^{2}\right) \\
\pi \text { - air } & \lambda_{\pi}=113.0\left(\mathrm{~g} / \mathrm{cm}^{2}\right) \\
\text { ratio } & \xi \equiv \lambda_{N} / \lambda_{\pi}=0.71
\end{aligned} \\
& \text { radiation length of the air } \quad X_{0}=37.1\left(\mathrm{~g} / \mathrm{cm}^{2}\right) \\
& \text { ratio } \quad \xi_{0}=\lambda_{N} / X_{0}=2.16 \\
& \text { critical energy of the air } \quad \epsilon=80.0(\mathrm{MeV})
\end{aligned}
$$

\section{Summary and discussion}

(i) We formulated three models, Model-0, Model1 and Model-2, for the energy spectrum of produced particles in multiple particle production (Table 4). Model-2 is based on the data of direct observation by cosmic-ray and accelerator experiments in the energy region of $10^{12} \sim 10^{14} \mathrm{eV}$. It indicates the violation of the Feynman scaling law both in the central region and in the forward region. Average inelasticity is $\langle K\rangle=0.5$ in Model-0 and in Model-1, but decreases with the incident energy in Model-2. Most of the nuclear interaction models, which are cmployed in recent simulations to follow the diffusion of cosmic rays in the atmosphere, lie between Model-1 and Model-2, from the view point of the energy distribution of produced particles.

(ii) Analytical expression is given for the size of the air shower, based on the formulated models of nuclear interactions. It cnables us to discuss effects of physical processes --. the charge exchange of the surviving pion, increasing cross section of hadron - air collisions, and the energy distribution of produced particles — to the air shower size. These processes are the major factors to govern the diffusion of high energy cosmic rays in the atmosphere. We obtained the following observations about the sizc of the extremely high energy air showers of $E_{0}=10^{20} \mathrm{eV}$.

(1) Effect of charge exchange process is almost constant $(x 1.13)$ over the whole depth in the atmosphere.

(2) Effect of increasing cross section is large ( $\times 2 \sim 3$ ) at mountain altitudes, but small $(\times 1.18)$ at the sea level.

(3) Effect of scaling violation of Model-1 is large $(x 2 \sim 3)$ at mountain altitudes, but small $(\times 1.23)$ at sea level.

(4) Effect of scaling violation of Model-2 is not negligible at any depth, i.e. $\times 0.6 \sim 0.4$ at mountain altitudes and $\times 0.22$ at the sea level.

(iii) The air shower size at sua level, expected by the present calculation, is tabulated in Table 8 for the incident proton of $E_{0}=10^{20} \mathrm{eV}$. In the table the effects of the charge exchange process and the increasing cross section are obtained by Fig. 15. To calculate the expected air shower size, to which the cffects of charge exchange probability and increasing cross section are included, we multiplied all the factors because the factors are near 1.0 .

(iv) M. Nagano et al. examined the method of energy determination of extremely high energy air showers, employed by AGASA experiment, by the simulation code of CORSIKA[26] (with QGSJET model). And they reached the conclusion that the method works well for the highest energy air 
Table 8

Air shower size at sea level, expected by the models, for the incident proton of $E_{0}=10^{20} \mathrm{eV}$

\begin{tabular}{lccc}
\hline & Model-0 & Model-1 & Model-2 \\
\hline size* & $5.0 \times 10^{10}$ & $6.2 \times 10^{10}$ & $1.1 \times 10^{10}$ \\
ratio to Model-0 & $(\times 1.0)$ & $(\times 1.23)$ & $(\times 0.22)$ \\
charge exchange & $\times 1.13$ & $\times 1.13$ & $\times 1.13$ \\
increasing cross section & $\times 1.18$ & $\times 1.18$ & $\times 1.18$ \\
\hline size (expected) & $6.7 \times 10^{10}$ & $8.3 \times 10^{10}$ & $1.5 \times 10^{10}$ \\
\hline${ }^{*}$ without the processes of increasing cross section and the charge exchange. \\
** with the processes of increasing cross section and the charge exchange.
\end{tabular}

showers.[28] The simulation gives $N_{e}=5.5 \times 10^{10}$ for the proton-induced air showers of $E_{0}=10^{20}$ $\mathrm{eV} .{ }^{12}$ We can see the following points by comparing the value with those in Table 7 .

(1) The value by the simulation is between those of Model-1 and Model-2. In this sense our calculation and the simulation are consistent each other, because we saw in Section 3 that the pseudorapidity density distribution by QGSJET model is between those by Model-1 and Model-2.

(2) If we take Model-1, the energy spectrum of highest energy air showers shifts to the left (toward lower energy) by a factor 1.5 .

(3) If we take Model-2, which is the best-fitted to the experimental data, the energy spectrum shifts to right (toward higher energy) by a factor 3.7.

Among the major factors to govern the cosmicray diffusion in the atmosphere - the energy distribution of produced particles, the charge exchange probability of the surviving pion and the increasing cross section of hadron-air collisions -- , one can see that the first one has the largest effect on the size of extremely high energy air showers. Hence we have to specify the energy distribution of produced particles in multiple particle production in more detail, in order to confirm the extremely high energy cosmic rays exceeding $10^{20}$ $e V$.

(v) The item (3) in the above paragraph (iv) makes the puzzle of extremely high energy cosmic rays more serious. That is, the energy spectrum of the primary cosmic rays, which is esti-

\footnotetext{
${ }^{12}$ We obtained $N_{e}=5.5 \times 10^{9}$ for the proton-induced air showers of $E_{0}=10^{19} \mathrm{eV}$, from the figure in Ref.[28], and multiplied it by $\mathrm{I} 0$.
}

mated by the highest energy air showers, extends beyond the Greisen-Kuzmin-Zatsepin cut-off energy $\left(\sim 10^{19} \mathrm{cV}\right)[29]$, which is one of the most important and interesting puzzles to be solved at present. [30]

Probably it is not irrelevant to conclude that the energy dependences of the scaling violation parameters in Model-2 are not valid at extremely high energy region. In other words Model 2 does not describe the extremely high energy air showers. This is due to the fact that Model-2 predicts small inelasticity at high energies. For example, the value is as small as 0.2 even at $E_{0}=10^{16} \mathrm{eV}$. (See Fig. 12.)

According to our previous analysis of attenuation mean free paths of hadron and $(e, \gamma)$ components [31], the inelasticity of $\langle K\rangle=0.5$ is compatible but smaller inclasticity is not compatible with the experimental data in the energy region of $10^{14} \sim 10^{16} \mathrm{eV}$. We will discuss in succeeding papers the cosmic-ray data in the encrgy region of $10^{14} \sim 10^{16} \mathrm{eV}$, presented in section 2 , and a model to describe all the available data consistently.

\section{Acknowledgment}

The author thanks the local organizing committee of the symposium for offering him an opportunity to give this talk and for holding very fruitful and enjoyable symposium.

The data, presented here, are obtained by the longstanding (more than 30 years !) BrazilJapan collaboration and by the Bolivia-BrazilJapan collaboration which is under way. Univer- 
sidade Estadual de Campinas, the host institute of the symposium, has been and is a member of the collaboration.

He is indebted to Prof. Edison H. Shibuya and Prof. Masanobu Tamada for valuable discussions of this work.

\section{REFERENCES}

1 C.M.G. Lattes, Y. Fujimoto, S. Hasegawa, Phys. Rep. 65 (1980) 65.

2 R. Feynman, Phys. Rev. Lett. 23 (1969) 1415.

3 T.K. Gaisser, R.J. Protheroe, K.E. Turver, T.J.L. McComb, Rev. Mod. Phys. 50 (1978) 859.

4 C.M.G. Lattes et al. Prog. Theor. Phys. Suppl. No.47 (1971) 1.

5 J. Nishimura, Handbuch der Physik Vol. XLVI/2 (Springer-Verlag) (1967) 1.

6 J.A. Chinellato et al., Prog. Theor. Phys. Suppl. No.76 (1983) 1.

7 F. Halzen and N. Yamdagni, Nucl. Phys. B190 (1987)213.

8 N. Arata, Nucl. Phys. B211 (1983) 189.

9 G.L. Alner et al. (UA5 Collaboration), Z. Phys. C33 (1986) 1; Nucl. Phys. B291 (1987) 445; Phys. Rep. Nos.5 and 6 (1987) 247.

10 S.I. Nikolsky, Proc. 3rd Sympo. on Cosmic Rays and Particle Physics, Tokyo (1984) (Inst. for Cosmic Ray Research, Univ. of Tokyo), 507.

11 L.T. Baradzei et. al., Nucl. Phys. B370 (1992) 365.

12 J.R. Ren et al., Phys. Rev. D38 (1988)1404.

13 N. Kawasumi et al., Phys. Rev. D53 (1996) 3534.

14 J. Phys. G 20 (1994) 487.

15 V.V. Arabkin, S.I. Nikolsky, K.V. Cherdyntseva and S.B. Schaulov, FIAN Preprint No.140 (1990) (Lebedev Physical Institute) (in Russian); Proc. 22nd Intern. Cosmic Ray Conf. (Dublin) (1991) Vol.4 pp.141, 269, 273.

16 C. Aguirre et al., Phys. Rev. D62 (2000) 032003; Talk by N. Kawasumi in this symposium.

17 T. Antoni et al., J. Phys. G 25 (1999) 2161.

18 E. Pare, T. Doke, M. Haugenauer, V. Innocente, K. Kasahara, T. Kashiwagi, J. Kikuchi,
S. Lazano, K. Masuda, H. Murakami, Y. Muraki, T. Nakada, A. Nakamoto, T. Yuda, Phys Lett. B242 (1990) 531.

19 R. Haar, C. Liapis, P. Karchin, C. Biino, S. Erhan, W. Hofmann, P. Kreuzer, D. Lynn, M. Medinnis, S. Palestini, L. Pesando, M. Punturo, P. Schlein, B. Wilkens, J. Zweizig, Phys. Lett. B401 (1997) 176.

20 M. Adams et al., Z. Phys. C39 (1988) 257.

21 J. Knapp, D. Heck, G. Schatz, Preprint of Forchungszentrum Karlsruhe, FZKA 5828 (1996).

22 G.J. Alner et al. (UA5 Collaboration), CERN-EP/86-213 (1986).

23 K. Werner, Phys. rep. 232 (1993) 87.

24 N.N. Kalmykov, S.S. Ostapchenko, Yad. Fiz. 56 (1993) 105; N.N. Kalmykov, S.S. Ostapchenko, Phys. At. Nucl. 56 (3) (1993) 346; N.N. Kalmykov, S.S. Ostapchenko, A.I. Pavlov, Bull. Russ. Acad. Sci. (Physics) 58 (1994) 1966.

25 R.S. Fletcher, T.K. Gaisser, P. Lipari, T. Stanev, Phys. Rev. D50 (1994) 5710; J. Engler, T.K. Gaisser, P. Lipari, T. Stanev, Phys. Rev. D46 (1992) 5013.

26 J.N. Capdevielle et al., Preprint of Kernforschungszentrum Karlsruhe KfK 4998 (1992).

27 J. Ranft, Phys. Rev. D51 (1995) 64.

28 M. Nagano, D. Heck, K. Shinozaki, N. Inoue, J. Knapp, Preprint of Forschungszentrum Karlsruhe, FZKA 6191 (1998).

29 S. Yoshida, Rapporteur Talk in 26th Int. Cosmic Ray Conf. (Salt Lake) (1999) AIP Conf. Proc. 516 p. 180 .

$30 \mathrm{~J}$. Cronin, the talk in this symposium.

31 A. Ohsawa, K. Sawayanagi, Phys. Rev. D49 (1992) 3128-3133. 


\title{
Hadron/Gamma Identification of Showers Observed by EC's
}

\author{
Y. Fujimoto ${ }^{a}$, V.V. Kopenkirı ${ }^{b}$, A. Ohsawac , M. Tarnadad \\ ${ }^{a}$ Advanced Research Center for Sci. and Eng., Waseda Univ., Shinjukı, Tokyo, 169 Japan \\ b Institute of Nuclear Physics, Moscow State University, 119899 Moscow, Russia \\ 'Institute for Cosmic Ray Research, University of Tokyo, Kashiwa, Chiba, 277-8582 Japan \\ ¿Faculty of Science and Engineering, Kinki University, Higashi-Osaka, Osaka, 577-8582 Japan
}

\section{Introduction}

Emulsion chamber (EC) is sensitive to high energy $(e, \gamma)$ 's and hadroms, incident upon the chamber, and can determine the positions and energies of these particles with high precision. It is used widely in the experiments to observe high energy cosmic rays, in particular in those at high mountains.

Objectives of mountain EC experiments are mainly to study nuclear interactions in the cnergy region exceeding those of accelerators and to study exotic events, such as Centauro, Chiron, etc. To study these subjects it, is important to identify the origin of observed showers, hadrons and $(e, \gamma)$ 's. For example, fraction of high energy hadrons and $(e, \gamma)$ 's in the air shower is one of the interesting parameters to study nuclear interactions which originate air showers. And one of the remarkable characteristics of Centauro events is a high fraction $(\sim 1.0)$ of hadron conterit.

In this report we present an algorithm to identify showers and some results which are obtained by applying the algorithm to experimental data.

\section{Showers observed by emulsion chamber}

\subsection{Emulsion chamber (EC)}

EC, a multiple sandwich of lead plates (usually $1 \mathrm{~cm}$ thick each) and sensitive layers (X-ray films, etc.), detects cascade showers which are produced in the chamber by the particles incident upon the chamber.

A cascade shower traverses several centimeters of lead, and therefore is recorded by several suc- cessive sensitive layers of EC. A cascade shower forms a small dark spot $(\sim 100 \mu \mathrm{m})$ on the Xray film after development. Darkness of the spot. is measured by a microphotometer with a slit of $200 \times 200 \mu \mathrm{m}^{2}$. EC detects spots with darkness cxcceding $\sim 0.1$, which corresponds to the shower energy of $\sim 1 \mathrm{TeV}$. The transition of the spot darkness values along the depth of the chamber $D(i)$ ( $i$ : $i$-th sensitive layer), called "(shower) transition curve", enables us to determine energy and starting depth of the shower, by comparing it with those calculated on the basis of the cascade shower theory, taking into account sensitivity curve of the X-ray film.

\subsection{Showers observed by EC}

(i) Gamma-showers and hadron-showers

Showers, originated by $(e, \gamma)$ 's, are called "gamma-showers". Their shower transition curves are characterized by a single peak at shallow layers of EC. On the other hand, a hadron also originates a cascade shower through its nuclear collision with lead, which is called a "hadron-shower". Most of hadron-showers have starting points deep in the chamber, because of long inelastic collision mean frec path of hadrons in the chamber $(\sim 18.0 \mathrm{~cm} \mathrm{~Pb})$. Sometimes the transition curve of a hadron-shower is not of a single peak but of multiple peaks, due to secondary collisions of the hadrons which are produced in the first collision.

(ii) Single-showers and family-showers

Observed showers are also classified into those of single arrival and those in a bundle. We call the former "single-showers" and the latter "family- 


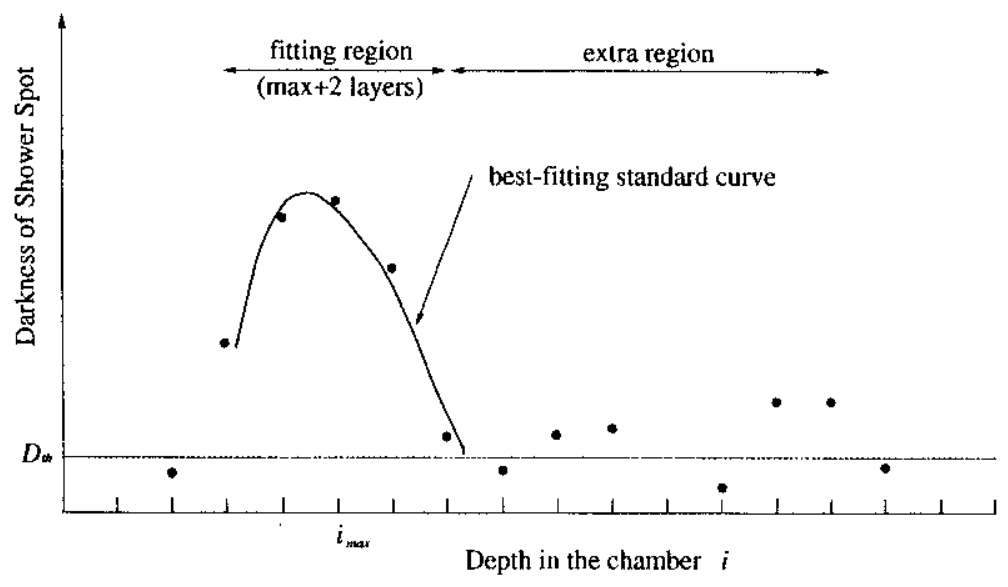

Figure 1. Illustration of fitting the standard curve to the data points

showers". A family, a bundle of gamma- and hadron-showers with the same direction of incidence, is produced by nuclear interaction(s) in the atmosphere.

Among hadron-showers, single-showers consist of those originated by nucleons and pions with roughly equal abundance, ${ }^{1}$ while family-showers are mainly due to pions.

\subsection{Gamma/hadron identification}

Gamma- and hadron-showers differ mainly in the starting depth and in the peaks of the shower transition curve. So far we have made hadronshower identification by the condition that the starting depth $\Delta t \geq 4(6)$ c.u. (cascade unit), and the number of hadron-showers with $\Delta t=0 \sim$ $4(6)$ c.u. is estimated statistically. ${ }^{2}$ This way is sufficient to discuss the intensities of $(e, \gamma)$ 's and hadrons. It is the study of exotic events that urges us to identify respective showers.

The following are the points which make identification devious.

(1) Development of cascade shower is sensitive only to the primary energy. In other words it is not so sensitive to the multiplicity of incident $\gamma$ rays if the total energy is the same.

\footnotetext{
${ }^{1}$ The intensities of nucleons and pions are approximately equal at mountain altitude.

${ }^{2}$ Hadron-showers with multiple peaks are rare because the
} chamber is not so thick so far.
(2) Fluctuation of the shower development due to stochastic nature of the cascade processes. In particular LPM effect causes large fluctuation of shower development in the energy region exceeding $50 \mathrm{TeV}$.

(3) Only the core region of the shower is observed by EC's, which makes shower fluctuation larger. For example, one Moliere unit, which is a measure of lateral spread of a shower, is $r_{0}=1.6 \mathrm{~cm}$ in lead, while the aperture of the microphotometer is only $200 \times 200 \mu \mathrm{m}^{2}$. (In other words X-ray film has low sensitivity.)

(4) Experimental errors mimic fluctuations.

\section{Fitting procedure}

The following is an algorithm to fit darkness data points $D(i)$ to the standard curves. (Fig. 1) (0) Standard curves by simulations.

Gamma-showers are produced for various primary energies $E_{0}$ and for various zenith angles of incidence $m \equiv \tan \theta$, by the simulation code of Ref.[1] which takes the chamber structure into account. The standard curves $D_{\text {std }}(i)$ and the dispersions $\sigma_{i}$ are obtained for various $E_{0}$ and $m$ by averaging them.

(1) The layer of maximum darkness $\Rightarrow i_{\max }$

(2) "Fitting region" of $\left[n_{1}, n_{2}\right]$, where $n_{1}$ is the layer of the first data point and $n_{2}=i_{\max }+2$. 
Table 1

Statistics of showers in the experiment and in simulations.

\begin{tabular}{lcc}
\hline (1) Experimental data & No. of registered showers & No. of showers for analysis \\
\hline Single-showers & 13290 & 469 \\
Family-showers & & 265 \\
\hline \hline (2) Simulated data & Conditions & No. of showers \\
\hline Gamma-showers & $E^{-2.8} d E(E \geq 10 \mathrm{TeV})$ & 720 \\
Hadron-showers & $E^{-2.8} d E\left(E_{a l l} \geq 10 \mathrm{TeV}\right)$ & 969 \\
\hline
\end{tabular}

(3) Calculation of deviation between the data points and $D_{s t d}(i)$, moving $E_{0}$ and the shower starting point $\Delta t$.

$$
\chi^{2} \equiv \sum\left[D(i)-D_{s t d}(i)\right]^{2} / \sigma_{i}^{2}
$$

The reduced $\chi^{2}$ is defined as $\mathcal{X}^{2} \equiv \chi^{2} /(n-2)$, where $n$ is the number of data points in the fitting region and consequently $n-2$ is a degree of freedom.

(4) The case of the minimum $\mathcal{X}^{2} \Rightarrow \mathcal{X}_{\min }^{2}$

(5) The standard curve of $\mathcal{X}_{\min }^{2} \Rightarrow$ Energy estimation $E_{I}$ of the shower in the fitting region.

(6) Calculation of the following quantitics outside the fitting region, called "extra region" hereafter.

$$
\begin{aligned}
& \Delta(i) \equiv \sum\left[D(i)-D_{\text {sld }}(i)\right] \text { (difference) } \\
& \delta(i) \equiv \Delta(i) / \sigma_{i} \text { (significance) } \\
& \sum \Delta(i)(\text { Track length } \\
& \quad \text { to estimate energy } E_{X} \text { in the extra region) } \\
& \chi_{e x}^{2} \equiv \sum \delta(i)^{2}
\end{aligned}
$$

(7) Estimation of the shower energy

$$
E_{\text {all }}=E_{I}+E_{X}
$$

\subsection{Statistics of showers}

At first we cxamine the showers which are obtained by thick lead chambers exposed at Pamirs. These data are suitable for examination of show. ers, because the chamber is uniform ( 59 sensitive layers at every $1 \mathrm{~cm} \mathrm{~Pb})$ and thick $(60 \mathrm{~cm} \mathrm{~Pb})$. The uniform chamber allows us to study shower devclopment in detail and the thick chamber to detect high energy hadrons with almost $100 \%$ efficiency. The showers in Table 1 are selected by the following criteria.

$$
\begin{aligned}
& D_{t h}=0.1 \text { (threshold darkness) } \\
& E_{t h}=10.0 \mathrm{TeV} \text { (threshold energy) } \\
& m=\tan \theta \leq 1.5\left(\theta \leq 56^{\circ}\right) \\
& \text { data points. } \geq 3 \\
& D \leq D_{m}\left(D_{m} \sim 4.0\right)
\end{aligned}
$$

Gamma- and hadron-showers are produced by simulations (See Table 1.), where the code of Ref.[1] is used for electromagnetic cascade processes and the code QGSJET[2] is assumed for hadron- $\mathrm{Pb}$ collisions. The energies of the incident particles are sampled from the power-type energy distribution $E^{-\beta-1} d E(\beta=1.8)$.

\section{Fitting procedure to simulation data of gamma-showers}

We examined the following points using gamma-showers from simulation.

(1) Optimum number of data points.

Varying the fitting range as $\left[i_{\max }-n, i_{\max }+\right.$ $n](n=1, \cdots, 5)$, we looked for the optimum number of data points for the fitting procedure. Our conclusion is that increase of data points does not necessarily mean good fit.ting or good energy estimation. $^{3}$

(2) Effect of threshold darkness $D_{t h}$

Decrease of $D_{t h}$ is equivalent to increase of data points. Hence we set $D_{t h}=0.1$.

(3) Validity of the fitting procedure

We applied the fitting procedure to the gammashowers produced by simulations without restricting the fitting region, and obtained the average value of reduced $\chi^{2}$ minimum as $\left\langle\mathcal{X}_{\min }^{2}\right\rangle=$ 1.08. This value means that the fitting procedure works satisfactorily, because $\left\langle\mathcal{X}_{\min }^{2}\right\rangle=1.0$ for an ideal case of $\chi^{2}$ minimum search.

(4) $E_{\text {all }}$ vs. $E_{0}$

Error in the encrgy estimation by our algorithm is $\sim 10 \%$.

\footnotetext{
${ }^{3}$ It is probably because neighboring data points, $D(i)$ and $D(i+1)$, are not independent completely. That is, large electron number at the $i$-th layer means again large number at the $(i+1)$-th.
} 
Table 2

Average value of reduced $\chi^{2}$ minimum

\begin{tabular}{lll}
\hline \multirow{2}{*}{ Experiment } & Single-showers & 2.72 \\
& Family-showers & 3.03 \\
\hline \multirow{2}{*}{ Simulation } & Gamma-showers & 0.80 \\
& Hadron-showers & 1.08 \\
\hline
\end{tabular}

\section{Fitting procedure in the fitting region}

The fitting procedure in the fitting region is applied to both data of the experiment and of the simulations. The average values of $\mathcal{X}_{\min }^{2}$ in Table 2 show that the simulation values are near 1.0 while those by experiment are far from 1.0 . It indicates that there is another source of shower fluctuation in experimental data.

Probably it is the experimental error in darkness measurements, which is not taken into account in the simulations and which would be reduced by smoothing the data or by using the average values of the neighboring two data points. After smoothing the $\mathcal{X}_{\text {min }}^{2}$ distribution of the experiment comes close to that of the simulation, particularly in small $\mathcal{X}_{\min }^{2}$ region. Or the value $\left\langle\mathcal{X}_{\min }^{2}\right\rangle=2.34$ becomes 1.42 after smoothing for single-showers. ${ }^{4}$

\section{Fitting procedure in the extra region}

Fitting procedure (the steps (6) and (7)) is applied to the extra region of the data from the experiment and simulations.

(1) $\chi_{e x}^{2}$ vs. $E_{X} / E_{0}$ by simulation data

The correlation between $\chi_{e x}^{2}$ and $E_{X} / E_{0}$ of gamma-showers and hadron-showers by simulations shows the following.

- Large $\chi_{e x}^{2}$ corresponds to $E_{X} / E_{0}>0$ and small $\chi_{e x}^{2}$ to $\left|E_{X} / E_{0}\right| \sim 0$, which signifies that larger value of $\chi_{e x}^{2}$ represents real successive showers due to hadron interactions.

- A criterion $\chi_{e x}^{2} \geq 20$ is good for hadron-shower identification.

(2) Integral distributions of $\chi_{e x}^{2}$ of the simulation data

\footnotetext{
${ }^{4}$ The number of events decreased from 469 to 410 , because some events do not fulfill the selection criteria after smoothing.
}

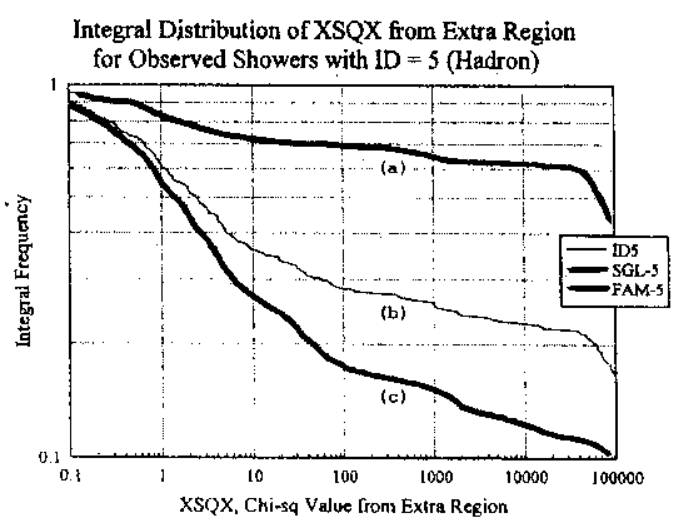

Figure 2. The distribution of $\chi_{e x}^{2}$ for the singleshowers (c) and for family-showers (a), both with the starting depth $\Delta t \geq 6$ c.u.

The $\chi_{e x}^{2}$ distributions of gamma-showers and hadron-showers by simulations are quite different, and show that the criterion of $\chi_{e x}^{2} \geq 20$ for hadron-shower identification works well.

(3) Integral distribution of $\chi_{e x}^{2}$ of the experimental data (hadron-showers)

Fig. 2 presents the $\chi_{e x}^{2}$ distributions of singleshowers and of family-showers, both with $\Delta t \geq 6$ c.u. The figure shows that both distributions are different in spite of the fact that all the showers are hadron-induced ones. Discussion on the origin of the observed difference, methodological or physical, is under way. One of possible descriptions is given by Tamada.[3]

\section{REFERENCES}

1 M. Okamoto et al., Nucl. Instr. Meth. A257 (1987) 155 .

2 N.N. Kalmykov et al., Bull. Russ. Acad. Sci. 58 (1994) 1966.

3 M. Tamada et al., Talk in this symposium. 


\title{
Characteristics of hadron-induced showers observed by the Pamir thick lead chambers
}

\author{
M.Tamada ${ }^{a}$ and $\Lambda$. Ohsawa $^{b}$ \\ ${ }^{a}$ Faculty of Science and Engineering, Kinki University, Osaka 577-8502, Japan \\ bInstitute for Cosmic Ray Research, University of Tokyo, Chiba 277-8582, Japan
}

\begin{abstract}
Structures of hadron-induced showers observed by the Pamir thick lead chambers are compared with sinulations using VENUS, QGSJET, HDPM and the modified UA5 model for hadron-makeus interactions. It is shown nome of the nodels of hadron-nuclens interactions used in the present analysis can describe all the characteristics of the observed two categories of hadron induced-showers, single-isolated and multi hadrons, at the same time.
\end{abstract}

\section{Introduction}

A series of exposures of lomogeneous-type thick lead emulsion chambers was carried out on the Pamir mountains by the Pamir collaboration experiment [1]. The homogeneous-type thick lead chamber has the advantage that we are able to observe the shower development of hadron-induced showers over a rarige of a few nuclear mean free paths, which enables us to study in detail characteristics of hadron-Pb interactions of hadron energy $E_{h_{.}} \geq 10^{2} \mathrm{TeV}$.

In Refs.[2] we discussed the inelasticity of hadron- $\mathrm{Pb}$ collisions at $10^{14} \mathrm{eV}$ by analyzing the structure of observed hadron-induced showers. A parameter $Z$ is defined as $Z \equiv E_{1}^{(\gamma)} / \sum E_{i}^{(\gamma)}$, where $E_{1}^{(\gamma)}$ is released energy at the first interaction and $\Sigma E_{i}^{(\gamma)}$ energy sum released at all the interactions during passage through the whole chamber. We compared the experimental distribution of $Z$ with those by the analytical calculations where various types of inelasticity distributions were assumed. We obtain $\left\langle k_{\text {inel }}^{h-P b}\right\rangle=$ $0.6_{-0.05}^{+0.03}$ for hadron- $\mathrm{Pb}$ collisions in the energy region of $E_{h} \simeq 10^{14} \mathrm{eV}$, appreciably smaller than usually considered. In the analysis the energies $E_{i}^{(\gamma)}$ were estimated by decomposing manually the observed shower transition into respective interactions. Therefore there still remains an ambiguity whether the experimental data can be compared directly with theoretical calculations or nol. In order to make a direct comparison possible, we carry out simulations for hadron-induced showers and apply just the same procedure both to experimental and simulated data.

\section{Pamir thick lead chambers}

The homogenious thick lead chamber is composed of 60 layers of lead plates of $1 \mathrm{~cm}$ thickness, equivalent to $\sim 3.5$ nuclear mean free paths. Russian R'T6-type X-ray films are inserted under every $1 \mathrm{~cm}$ lead plate except for the first $2 \mathrm{~cm}$ of lead. 'The darkness detection threshold, $D_{t h}$, of the shower spot is $\sim 0.2$, which corresponds to a shower energy of $2 \sim 3 \mathrm{~K} \mathrm{eV}$ approximately. Details of the chambers are given in Ref. [1].

A shower detected in the chamber is either a single-isolated shower, which is not accompanied by any other parallel shower of energy above the detection threshold, or a menber of a bundle of parallel showers with the same arrival direction, which we call a cosmic-ray "family"

\section{Simulations}

We use the following four models for hadron$\mathrm{Pb}$ interactions;

(1) VENUS [3], (2) QGSJET [4]: both of which are based on the Gribov-Regge theory of multiple Pomeron exchanges.

(3) HDPM [5] : a semi-enpirical model extrapolating experimental data based on the Dual Multichain Parton model and

(4) a phenomenological UA5 algorithm [6] modified for hadron-nucleus interaction using a geometrical approach. 
We calculate the development of nuclear and electromagnetic cascades in lead initiated by hadron$\mathrm{Pb}$ interactions under the following assumptions: (1) the energy spectrum of hadrons arriving at the chamber is of power-law lype, $I(\geq E) \propto E^{-\beta}$ with $\beta=1.8$ for single-isolated hadrons and $\beta=1.2$ for hadrons in a family,

(2) the zenith angle distribution of arriving hadrons is $I(\leq \cos \theta) \propto(\cos \theta)^{-8}$ and

(3) the total thickness of the chamber is $60 \mathrm{cmPb}$ and the sensitive layers are inserted under every $1 \mathrm{cmPb}$.

The interaction mean free path of hadron- $\mathrm{Pb}$ interactions is assumed to be energy dependent, e.g., $\Lambda_{\text {coll }}^{p-P b}(E)=15.9 \mathrm{cmPb}$ and $\Lambda_{\text {coll }}^{\pi-P b}(E)=$ $17.5 \mathrm{cmPb}$ at $E=10^{14} \mathrm{eV}$.

Protons and pions of $E_{h} \geq 30 \mathrm{TeV}$, respectively, are sampled from the above energy spectra, and all hadrons, produced in the collisions during passage through the chamber, are followed until their energy falls below $80 \mathrm{GeV}$ or they leave the chamber. For $\gamma$-rays of $E_{\gamma} \geq 1 \mathrm{GeV}$, which are mainly decay products of $\pi^{0}$ s, we further calculate the three-dimensional electromagnetic cascade development using the Monte-Carlo code formulated by M. Okamoto and T. Shibata [7]. in which the LPM effect is also taken into accounts. Electrons and photons are followed until their energies fall below $1 \mathrm{MeV}$. The electron number density, $\rho_{e}$, is converted to the local spot darkness, $d$, of $\mathrm{X}$-ray film, by using the characteristic relation for the Russian R'T'6-type X-ray fitm, and finally we obtain the transition curve of the spot darkness $D$ vs. depth ' $T$ ' throughout the chamber.

\section{Estimation of $\Sigma E_{\gamma}$ and $E_{1}^{(\gamma)}$}

\subsection{Total released energy $\Sigma E_{\gamma}^{\prime}$}

The total observed energy of the hadron, $\Sigma E_{\gamma}$, released in form of $\gamma$-rays during the passage through the chamber is estimated by the sum, $\Sigma D_{i}$, of the shower-spot darknesses $D_{i} \geq D_{\min }$ in its longitudinal development. Here we set $D_{\min }=0.3$. We observe a fairly good correlation between $\Sigma E_{\gamma}$ and $\Sigma D_{i}$ as shown in Fig.1 for the QGSJET model. 'The other three models give similar results.

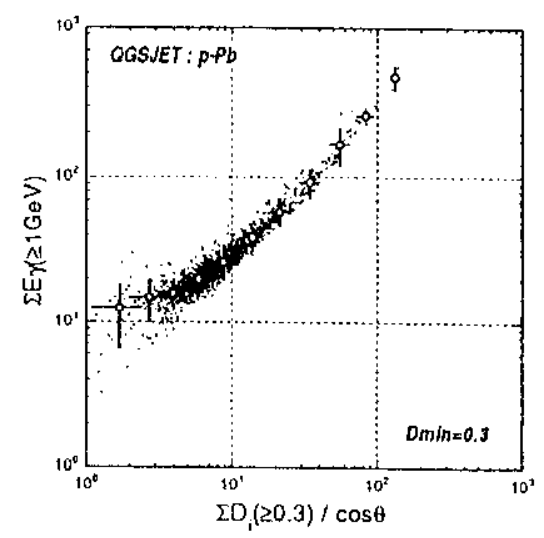

Figure 1. Diagram on $\Sigma E_{\gamma}$ and $\Sigma D_{i}\left(D_{i} \geq\right.$ $0.3) / \cos \theta$ for $p-P b$ interactions. $\theta$ is zenith angle of the incident badron. Open circles show average dependence.

\subsection{Energy $E_{1}^{(\gamma)}$ released in the first inter- action}

In order to extract the energy released in the first interaction from the whole shower transition curve of the event, the following fitting procedure is applied for the first 6 layers in which the spot darkness exceeds $D \geq 0.3$. From a the set of standard transition curves $D^{s t d}$ vs. $T$, calculated for showers of electron-positron pair origin taking into account the exact clamber structure, the best fit is selected by choosing the energy value, $E_{1}^{(\gamma)}$, and the first pair-creation depth, $\Delta T$, by a computer algorithm employing the gradient descent method for a search of the chi-square mininum. We also calculate the deviation of the observed shower transition from the best-fit by $\delta \equiv \Sigma\left(D_{i}-D_{i}^{s t d}\right) / \sigma_{i}$, where the summation is made for the layers beyond the fit range and $\sigma_{i}$ is the dispersion of darkiness of the standard transition curve at the $\mathrm{i}$-th layer. A large value of the deviation $\delta$ indicates that there are contributions from successive interactions.

lig.2 shows a distribution of $E_{1}^{(\gamma)} / E_{1}^{\text {(rue }}(\gamma)$ where $E_{1}^{\text {true }}(\gamma)$ is the true energy released into $\gamma$-rays at the "first interaction". The distribu- 
tion has a clear peak at $E_{1}^{(\gamma)} / E_{1}^{\text {true }}(\gamma)=1.2$, indicating that the present procedure works but overestimates the energy released in the "first interaction" by $\sim 20 \%$ on average. The same fit procedure is also applied to $\gamma$-ray-induced pure electromagnetic cascades. The estimaled energy is very close to the true energy in this case (Fig.2).

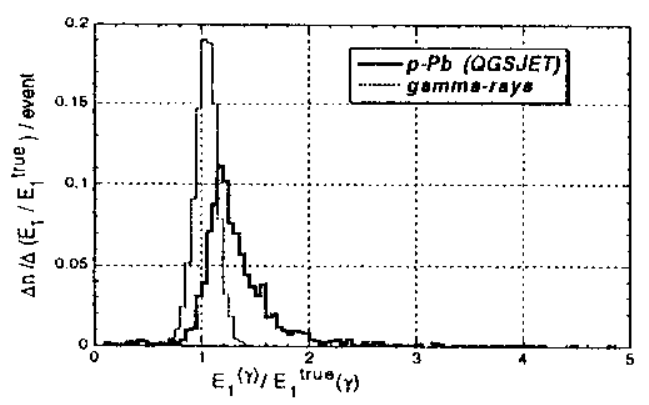

Figure 2. Distribution of $E_{1}^{(\gamma)} / E_{1}^{\text {true }}(\gamma)$, for $p-$ $P b$ interactions using the QGSJEI model (solid histogram). The dotted histogram is for $\gamma$-ray induced pure electromagnetic cascades.

\section{Selection of the events}

Emulsion chambers detect both $(e, \gamma)$-induced and hadron-induced slowers. Here we identify a shower as a hadron-induced one if $\Delta T^{\prime} \geq 4$ $\mathrm{cmPb}$ or $\delta \geq 50$. According to the simulation, almost all $(e, \gamma)$-induced slowers are rejected by these criteria. We demand further that the darkness at shower maximum, $D_{\max }$, in the shower transition is less than 3 , because our standard procedure of photometric measurement is limited up to $D=3 \sim 3.5$, and that the sum of spot darknesses, $\Sigma D_{i}\left(D_{i} \geq 0.3\right) / \cos \theta$, is larger than 8 , which corresponds to a total released energy $\Sigma E_{\gamma} \geq 20-30 \mathrm{TeV}$. In the Pamir thick lead chamber of $66 \mathrm{~m}^{2}$.year exposure, we have 139 showers which satisfy the above criteria. Among them 54 are found as members of atmospheric families and the other 85 are isolated ones. The same selection criteria are also applied to simulated hadroninduced sliowers.

\section{Distribution of $Z \equiv E_{1}^{(\gamma)} / \Sigma E_{\gamma}$}

Applying the procedure described in the section 4 , we obtain $E_{1}^{(\gamma)}$ and $\sum E_{\gamma}$ for the above selected events both in the experiment and in the simulations. We re-define the inelasticity-related parameter $Z$ by $Z \equiv E_{1}^{(\gamma)} / \Sigma E_{\gamma}$. Fig. 3 shows the experimental distributions of $Z$ for single-isolated hadrons and Fig. 4 for hadrons in families. On average hadrons in families have a smaller value of the parameter 7 . The distributions are compared with those of simulated hadron-induced showers. In Fig.3 we assume that half of the incident hadrons are protons and the other half are pions both of which have the integral energy spectrum of the power index $\beta=1.8$. On the other hand, in lig. 4 we assume that all incident hadrons are pions with an energy spectrum of spectral index $\beta=1.2$. The dependence of the $Z$-distribution on the power index $\beta$ and the nature of incident particles, however, is found to be small.

As is seen in the figure, the experimental distribution of single-isolated hadrons is described well by VENUS, QGSJE' ${ }^{\prime}$ and the modified UA5 model, all of which give an average inelasticily of $\left\langle K^{\prime}\right\rangle=0.7 \sim 0.8 .{ }^{1}$ But the data deviate from the HDPM nodel which predicts a smaller inelasticity $\langle K\rangle=0.5 \sim 0.6$. For hadrons in families, on the contrary, more events populate the region of smatler $Z$, and the experimental distribution is close to that of the fll)PM model and not compatible with those of the other three models. Thus none of the models can describe simultaneously the experimental $Z$-distribution of the two categories of hadrons. 'The $Z$-distributions of single-isolated hadrons and hadrons in farnilies can be distorted by the poor accuracy in tracking of the shower spots. However, according to the examinations, these cannot be made responsible for the observed difference of the $Z$ distribution

\footnotetext{
Here the inclasticity is given by $K \equiv 1-x_{l}$ where $x_{l}$ is defined by the energy fraction carried by the highest energy baryon ( $\pi^{ \pm}$meson) in $p\left(\pi^{ \pm}\right)-P b$ interactions.
} 

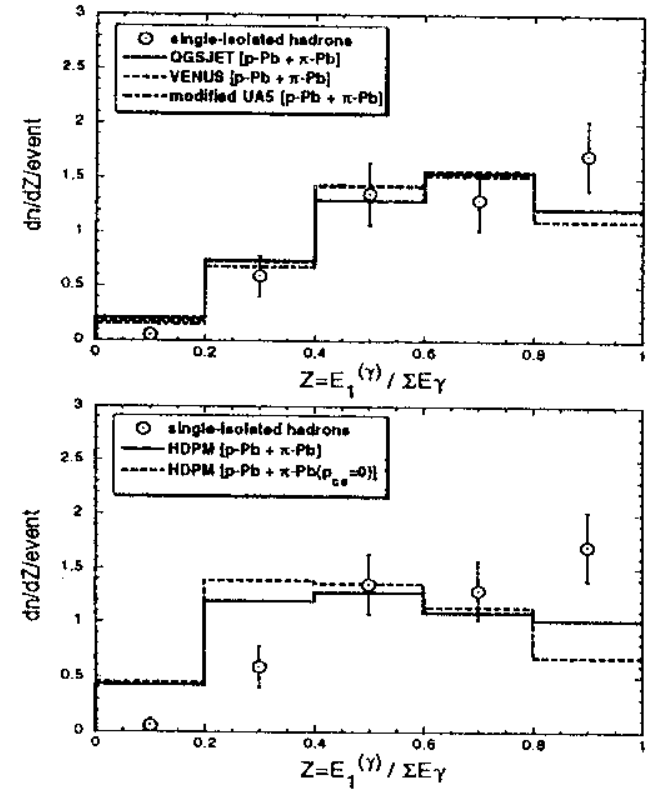

Figure 3. Distribution of $Z \equiv E_{1}^{(\gamma)} / \Sigma E_{\gamma}^{\gamma}$ for single-isolated hadrons. The histograms are those for simulated hadron-induced showers. $p_{c e}=0$ means no charge exchange processes in pion- $\mathrm{Pb}$ interactions.

in the two categories of incident hadrons. One of the possible explanations of the observed diffrence between single-isolated hadrons and hadrons in families is to assume the existence of hadron bundles in high-energy cosmic-ray families. That is, if there exists a hadron bundle in which the mutual distance of the constituent hadrons is extremely small, i.e. less than $\sim 1 \mathrm{~mm}$, we would possibly misidentify two independent hadron-induced showers as those produced by successive interactions. It would produce a smaller value of the parameter $Z$ even if the average inelasticity is as large as that of single-isolated hadrons. The experimental data, however, require the number of those hadron bundles to be $3 \sim 4$ times more frequent than expected simply by chance. Extraordinary correlated hadron-bundles remind us of exotic shower-clusters, named "mini-clusters", in which hadrons and $\gamma$-rays are closely corre-
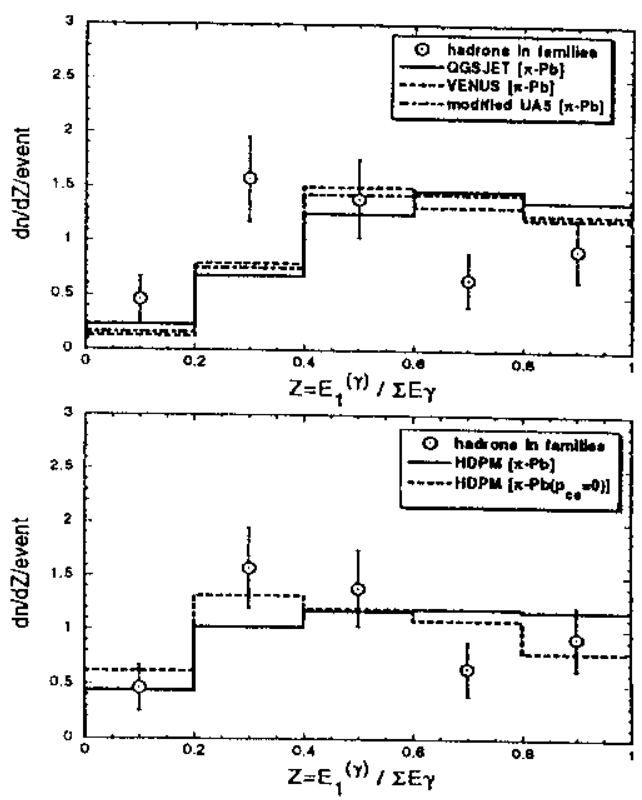

Figure 4. Distribution of $Z$ for hadrons in families. The histograms are those for simulated hadron-induced showers.

lated, which are observed in high-energy cosmicray families detected in carbon-chambers of the Chacaltaya and Pamir experiments.

\section{REFERENCES}

1 Chacaltaya and Pamir Collaboration (T.Arisawa et al.); Nucl. Phys. B 424 (1994) 241

2 C.R.A.Augusto, el al.: Phys. Rev. D 61, (1999) 012003

3 K.Werner, Phys. Rep 232 (1993) 87

4 N.N.Kalmykov, S.S.Ostapchenko\& A.l.Pavlov: Bull. Russ. Acad. Sci. (physics) 58 (1994) 1966,

5 J.N.Capdeville: J. Phys. G: Nucl. Part. Phys. 15 (1989) 909

6 UA5 Collaboration (G.L.Alner et al.): $\mathrm{Nucl}$. Phys. B 291 (1987) 445

7 M.Okamoto \& T.Shibata: 1987, Nucl. Instr. Meth. A257 (1987) 155 


\title{
A study on the penetrating nature of gamma- and hadron- induced cascade showers in the two-storey emulsion chambers
}

\author{
M.Tamada ${ }^{a}$ \\ aFaculty of Science and Engineering, Kinki University, Osaka 577-8502, Japan
}

Penetrating nature of the cascade showers observed in the Chacaltaya two-storey chamber is compared with that of simulated $\gamma$-ray- induced and hadron-induced cascade showers. It is shown that around $40 \%$ of the penetrating showers are neither $\gamma$-ray-induced ones nor hadron-induced ones. A possible explanation is given in connection with 'mini-clusters'.

\section{Introduction}

The Chacaltaya and Pamir emulsion chamber experiments have shown that there exist unusual phenomena which are not yet observed in the present accelerator experiments from the analysis of high energy cosmic-ray families, a bundle of electromagnetic particles and hadrons produced in the nuclear and electromagnetic cascade process in the atmosphere.[1] Those are called 'Centauro-species', multiple hadron production without association of $\pi^{0}$-mesons. It is also reported that the nature of secondary particles is different from that of ordinary hadrons in those unusual phenomena. In Ref.[2] we discussed about unusual behaviour of cascade development of the high energy showers in high-energy cosmic-ray families observed by Chacaltaya twostorey chamber no.19. We studied in detail how the shape of the cascade transition of the observed showers, which penetrate from the upper chamber down to the lower chamber, deviate from that of standard electromagnetic cascades expected in the uniform lead chamber. We found frequent existence of strong penetrating showers which became rejuvenated after passing through the target layer. The results were discussed in connection with 'mini-clusters', clusters which consist of extraordinarily correlated $\gamma$-rays and hadrons. $[1,3]$ Here we discuss the issue again comparing the shape of the cascade showers by those initiated by $\gamma$-rays and also by hadron interaction taking into accounts the exact structure of the Chacaltaya two-storey chamber.

\section{Experimental data}

Fig.1 shows the basic structure of Chacaltaya two-storey chamber no.19. The chamber consists of the upper chamber of $6 \mathrm{cmPb}$, the target layer of $23 \mathrm{~cm}$ carbon (petroleum pitch), wooden support of $5 \mathrm{~cm}$ thick, the air gap of $158 \mathrm{~cm}$ height and the lower chamber of $8.4 \mathrm{cmPb}$. Four sensitive layers (X-ray film and nuclear emulsion plate) are inserted in the upper chamber and eight sensitive layers in the lower chamber. The chamber no.19, the area of which is $44.4 \mathrm{~m}^{2}$ in the upper chamber and $32.4 \mathrm{~m}^{2}$ in the lower chamber, was exposed 677 days at Mt. Chacaltaya. Showers detected in the upper chamber are mainly $(e, \gamma)$ induced ones with small admixture of hadroninduced ones. Showers detected in the lower chamber, on the other hand, are those initiated by nuclear interactions in the target layer $(C-j e t s)$ and in the lead plates of the lower chamber itself $(\mathrm{Pb}-j e t-$ lower $)$. In the present analysis we pick up showers in the high energy cosmic-ray families with total visible energy grater than 100 $\mathrm{TeV}$ observed by the Chacaltaya chamber no.19.

\section{Penetrating cascade showers}

Some of the showers observed in the upper chamber can be followed down into the lower chamber. We define it as a penetrating shower when its spot darkness, $D$, is larger than 0.2 in at least two layers in the upper chamber and at least one layer in the lower chamber. A typical example of the shower transition of the penetrating shower is shown in Fig.2. In Table 1 we summarise the number of showers with visi- 


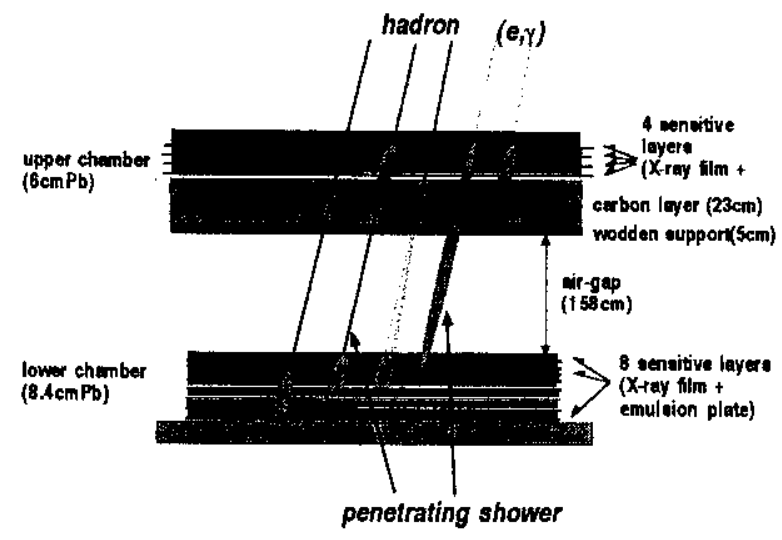

Figure 1. Illustration of Chacaltaya two-storey chamber no.19.

ble energy $E(\gamma) \geq 10 \mathrm{TeV}$ observed in the upper chamber, those observed only in the lower chamber $(C-j e t s, P b-j e t s-l o w e r)$ and those of penetrating. Among 205 showers observed in the upper chamber, 85 penetrate into the lower chamber.

\section{Simulations}

We calculate a development of cascade showers in the two-storey chamber, of the structure just same to the Chacaltaya chamber no.19, initiated from a pure $\gamma$-ray and from a hadron-Pb (C) interaction.

\subsection{Hadron-induced showers}

We use two different models for hadron-nucleus interactions, one is QGSJET [4] based on the Gribov-Regge theory of multiple Pomeron exchanges and the other is phenomenological UA5 algorithm [5] modified for hadron-nucleus interaction using a geometrical approach. In the nuclear cascade all hadrons, produced in the collisions during passage through the chamber, are followed until their energy falls below $80 \mathrm{GeV}$ or they leave the chamber. The interaction mean free path of hadron-nucleus interactions is assumed to decrease with increasing interaction energy, e.g.,

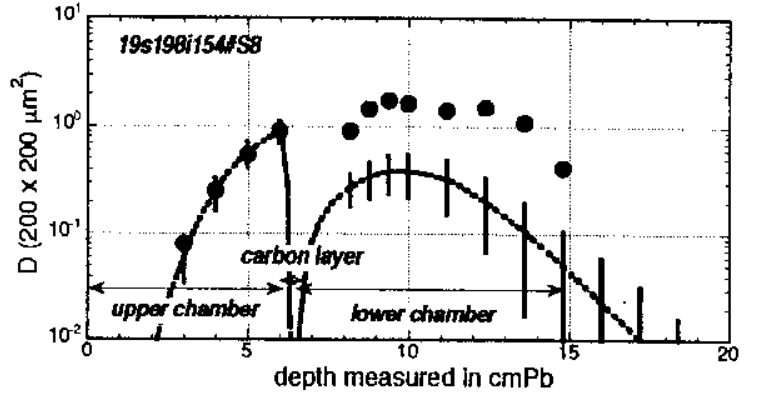

Figure 2. An example shower transition on spot darkness of the penetrating showers observed in Chacaltaya chamber no.19. Dotted curve is the best fit described in the text. Vertical bars are dispersion $\sigma$ of the standard transition curve.

$\Lambda^{p-P b}(E)=15.9 \mathrm{cmPb}, \Lambda^{\pi-P b}(E)=17.5 \mathrm{cmPb}$, $\Lambda^{p-C}(E)=62.4 \mathrm{cmC}$ and $\Lambda^{\pi-C}(E)=79.4 \mathrm{cmC}$ at $E=10^{14} \mathrm{eV}$.

For $\gamma$-rays of $E_{\gamma} \geq 1 \mathrm{GeV}$, which are mainly decay products of $\pi^{0}$ s produced in the collisions, we further calculate the three-dimensional electromagnetic cascade development in the chamber using the Monte-Carlo code formulated by M. Okamoto and T. Shibata [6], in which the LPM effect is also taken into accounts. Electrons and photons are followed until their energies fall below $1 \mathrm{MeV}$. The electron number density, $\rho_{e}$, is converted to the local spot darkness, $d$, of Xray film, by using the characteristic relation for the N-type X-ray film, and finally we obtain the transition curve of the spot darkness $D$, measured by a $200 \times 200 \mu \mathrm{m}^{2}$ slit, vs. depth $T$ throughout the chamber. The experimental error of the measurement of spot darkness $D$ is also taken into accounts by adding noise $\Delta D$ in each spot darkness where $\Delta D$ is sampled from Gaussian distribution with $\sigma_{D}=0.1 D$. Protons and pions of $E_{h} \geq 20 \mathrm{TeV}$ are sampled from the energy spectrum $I\left(\geq E_{h}\right) \propto E_{h}^{-1.2}$ and from zenith angular distribution $I(\leq \cos \theta) \propto(\cos \theta)^{-8}$.

\section{2. $\gamma$-ray-induced showers}

We also calculate electromagnetic cascade development in the chamber initiated by $\gamma$-rays us- 
Table 1 Number of high energy showers, $E(\gamma) \geq$ $10 \mathrm{TeV}$, observed in the chamber no.19.

\begin{tabular}{l}
\hline $\begin{array}{l}\text { atmospheric families of } \\
\Sigma E(\gamma) \geq 100 \mathrm{TeV}\end{array}$ \\
$\begin{array}{l}\text { (a) no. ovents } \\
\text { observed in the upper } \\
\text { chamber }\end{array}$ \\
\hline $\begin{array}{l}\text { (b) penetrating } \\
\text { (c) cxpected no. of pene- }\end{array}$ \\
$\begin{array}{l}\text { trating } \gamma \text {-rays } \\
\text { (d) non- } \gamma \text {-ray origin }\end{array}$ \\
$\begin{array}{l}\text { (e) no. of showers of ob- } \\
\text { served only in the lower } \\
\text { chamber ( } C \text { - jets, } P b-\end{array}$ \\
jets-lower) \\
\hline $\begin{array}{l}\text { (f) expected no. } \\
\text { penetrating showers of } \\
\text { hadronic origin }\end{array}$ \\
\hline $\begin{array}{l}\text { (g) no. of anomalous pen- } \\
\text { etrating showers }\end{array}$ \\
\hline
\end{tabular}

ing the above mentioned Monte-Carlo code assuming the energy spectrum and zenith angle distribution of the $\gamma$-rays arriving at the chamber as $I\left(\geq E_{\gamma}\right) \propto E_{\gamma}^{-2}$ and $I(\leq \cos \theta) \propto(\cos \theta)^{-8}$ respectively.

\section{Penetrating probability}

According to the simulations, the penetrating probability of $\gamma$-ray-induced showers of $E_{\gamma} \geq 10$ $\mathrm{TeV}$ is found to be $0.23 \pm 0.01$. If we assume all the 205 showers observed in the upper chamber are $\gamma$-ray-induced ones, the expected number of penetrating showers of $\gamma$-ray origin is then obtained as $47 \pm 4$. That is, around one half of the observed penetrating showers can be considered to be $\gamma$-ray origin but the other half $(38 \pm 10)$ are non- $\gamma$-ray origin. Possible origin of those which we can consider are hadron-induced showers. Now let's estimate the number of hadroninduced penetrating showers. All the showers detected only in the lower chamber can be considered to be hadron-induced ones $(C-j e t s$ and
$P b-j e t s-$ lower $)$. In the simulation calculations of hadron-induced showers we know that the ratio between the number of penetrating hadroninduced showers and that of showers which are observed only in the lower chamber. The results are summarized in Table 2. As is seen in the table, the ratio is around 0.4 on the average. In the experiment, there are 23 showers which are observed only in the lower chamber. Then we can obtain the expected number of hadron-induced penetrating showers to be $9 \pm 2$. Among 38 penetrating showers of non- $\gamma$-ray origin, only 9 can be due to hadronic interaction in the upper chamber. Then we can conclude the rest $29 \pm 10$ penetrating showers ( $\sim 34 \%$ of all the penetrating showers) are neither $\gamma$-ray-induced nor hadroninduced showers.

Table 2 Number of hadron-induced showers $\left(E_{h}(\gamma) \geq 10 \mathrm{TeV}\right)$.

\begin{tabular}{|c|c|c|c|c|}
\hline & \multicolumn{2}{|c|}{ QGSJET } & \multicolumn{2}{|c|}{ modified UA5 } \\
\hline incident & pion & proton & pion & proton \\
\hline (1) penetrating & 193 & 208 & 192 & 236 \\
\hline $\begin{array}{l}\text { (2) visible only in } \\
\text { the lower chamber }\end{array}$ & 550 & 486 & 527 & 545 \\
\hline ratio $=(1) /(2)$ & 0.35 & 0.43 & 0.37 & 0.43 \\
\hline
\end{tabular}

In each set of the calculations, 2,000 particles are sampled from the spectra described in Section 4.1.

\section{Shape of the shower transition of the penetrating showers}

Here we study how the shape of the shower transition of the penetrating showers differ from that of ordinary $\gamma$-ray induced cascades. ${ }^{1}$ The following fitting procedure is applied for the lay-

\footnotetext{
${ }^{1}$ In the Chacaltaya chamber no.19, full data of spot darkness of the penetrating showers are available at present for a half of the chamber which were measured in Japan. Hence the analysis of the shape of the shower transition is made using 56 penetrating showers in 22 cosmic-rays families.
} 
ers of the upper chamber in which the spot darkness exceeds $D \geq 0.2$. From a set of standard transition curves $D^{\text {sid }}$ vs. $T$, calculated for showers of $e^{-} e^{+}$-pair origin, taking into accounts the exact structure of the two-storey chamber no.19, the best fit is selected out by choosing the energy value $E_{\gamma}^{f i t}$ and the first pair-creation depth $\Delta T$ by a computer algorithm employing the gradient descent method for a search of the chi-square minimum. We then calculate the deviation of the observed shower transition in the lower chamber from the best-fit by $\delta \equiv \Sigma\left(D_{i}-D_{i}^{s t d}\right) / \sigma_{i}$, where the summation is made for the layer in the lower chamber, $\sigma_{i}$ is the dispersion of the standard curve at the i-th layer. Fig. 3 shows integral distribution of $\delta$ for the events with $E_{\gamma}^{f i t} \geq 5 \mathrm{TeV}$. The same procedure is applied to the simulated penetrating showers of $\gamma$-ray origin and of hadronic origin. In the figure we show the expected distributions in the case that penetrating showers consists of a mixture of $\gamma$-ray-induced and pioninduced ones. As is scen in the figure, the experimental distribution is well described by assuming about $40 \% \sim 50 \%$ of penetrating showers are hadron-induced ones, i.e., non- $\gamma$-ray origin. The estimated fraction of hadron-induced penetrating showers does not depend much on the assumed model of hadron-nucleus interaction and on the nature of the incident particle.

\section{Discussions}

We have shown that around $34 \%$ of penetrating showers ( $\sim 14 \%$ of the showers in the upper chamber) observed in the Chacaltaya two-storey chamber no. 19 are neither $\gamma$-ray-induced nor hadroninduced showers. One of the possible explanations is to assume an existence of extremcly collimated pair of a $\gamma$-ray and a hadron. That is, if the mutual distance between a $\gamma$-ray and a hadron is extremely small, e.g., less than $\sim 1 \mathrm{~mm}$, and the $\gamma$-ray-induced showers is observed in the upper chamber and the hadron-induced shower is observed in the lower chamber, we would possibly misidentify those two as a penetrating shower. If a $\gamma$-ray make electromagnetic interactions in the atmosphcre, we can observe collimated several $(e, \gamma)$-particles and a hadron as a 'mini-cluster'

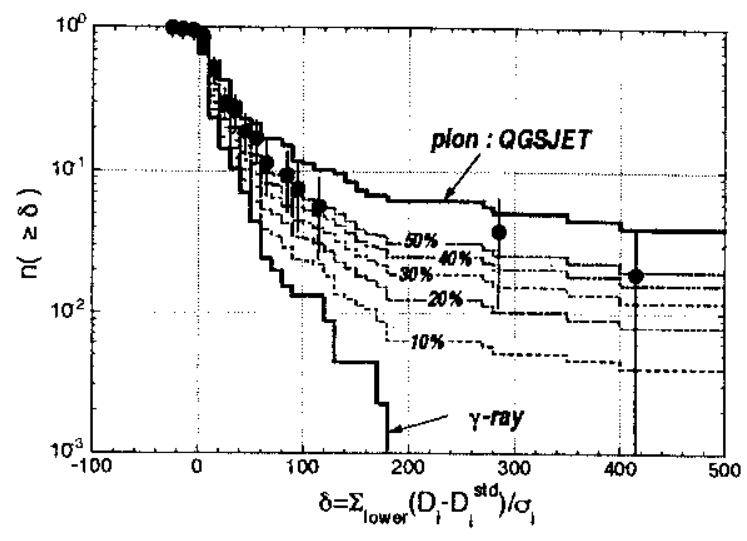

Figure 3. Integral distribution of $\delta \equiv \Sigma\left(D_{i}-\right.$ $\left.D_{i}^{\text {std }}\right) / \sigma_{i}$ for the penetrating showers. Thin histograms are for the case of mixture of pioninduced and $\gamma$-ray induced showers. Percentage attached to the histograms show the assumed fraction of the pion-induced penetrating showers.

which are often found in the exotic events. Possible existence of hadron-bundles in which the mutual distance of the constituent hadrons is extrmely small is also discussed in the analysis of shower transition of high-energy hadronic showers observed in the Pamir thick lead chamber[7, 8].

\section{REFERENCES}

1 Chacaltaya and Pamir Collaboration (L.T.Baradzei et al.); Nucl. Phys. B 370 (1992) 365

2 Y.Funayama \& M.Tamada : J. Phys. Soc. Japan 55, (1986) 2977

3 M.Tamada \& Y.Funayama : J. Phys, Soc. Japan 55, (1986) 2996

4 N.N.Kalmykov, S.S.Ostapchenko \& A.I.Pavlov; Bull. Russ. Acad. Sci. (physics) 58 (1994) 1966,

5 UA5 Collaboration (G.L.Alner et al.): Nucl. Phys. B 291 (1987) 445

6 M.Okamoto \& T.Shibata: Nucl. Instr. Meth. A257 (1987) 155

7 M.Tamada \& A.Ohsawa: Nucl. Phys. B 581 (2000) 73

8 M.Tamada \& V.V.Kopenkin: Nucl. Phys. B 494 (1997) 3 


\title{
Study of Hadronic Component in Air Showers at Mt. Chacaltaya
}

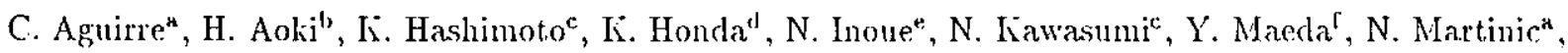 \\ N. Ohmori', A. Ohsawag, K. Shinozakie, M. Tamada ${ }^{\mathrm{h}}$, R. Ticona a and I. Tsushinac: \\ "Instituto de Investigaciones Fisicas, Universidad Mayor de San Andres, La Pa7, Bolivia \\ 'Faculty of Science, Soka University, Hachioji, Tokyo, 192-8577 Japan \\ "Faculty of Education, Yamanashi University, Liofu, 400-8510, Japan \\ "Faculty of Engineering, Yamanashi University, Kiofu, 400-8511, Japan \\ "Faculty of Science, Saitama University, Urawa, 388-8570 Japan \\ 'Faculty of Science, Kochi University, Kochi, 780-8520 Japan \\ BInstitute for Cosmic Ray Research, University of Tokyo, Liashiwa, 277-8582 Japan \\ "Faculty of Science and Technology, Kinki University, Higashi-Osaka, 577-8502 Japan
}

An experiment of an air shower array, a hadron calorimeter $\left(8 \mathrm{~m}^{2}\right)$ and an cmulsion chamber $\left(8 \mathrm{~m}^{2}, 15 \mathrm{~cm}\right.$ $\mathrm{Pb})$ is under way at $\mathrm{Mt}$. Chacaltaya $(5200 \mathrm{~m}$ above sea level, Bolivia), in order to study the hadron interactions and the primary cosmic rays in the energy region exceeding $10^{15} \mathrm{eV}$. The number of hadronic component in the air shower, which is detected by the hadron calorimeter, is not compatible with those by simulations, indicating that violation of the Feymman scaling law is stronger at $10^{60} \mathrm{eV}$ than the one assumed in the sinulations. The average mass number of the primary costnic rays, estimated from the distribution of the number of hadrons in the air shower, is $\langle\ln A\rangle=2.8 \pm 0.5$ at $10^{16} \mathrm{eV}$.

\section{Introduction}

An experiment of an air shower array, a hadron calorimeter and an emulsion chamber is being carried out. at. Mt. Chacaltaya $(5200 \mathrm{~m}$, Bolivia). The enulsion chanber deterts high energy particles in the air shower and those are called "fannily". In this way the experiment. simultaneously supply data of the electron component in the air shower together with those of high energy particles in the air shower core. Air shower experiments and emulsion chamber experiments, which have been carried independently so far, accumulate a large amount of data, respectively. Hence it is interesting and important to briclge the data by both experiments and a large scale of a new experimental set-11p is not needed for this purpose. The present experiment enables us to study the structure of the air showers, the nuclear interactions and the primary cosmic rays in the energy region of $10^{15} \sim 10^{17} \mathrm{eV}$. Detailed knowledge of the air shower development is important, in particular at present because there are experiments, running and as a project, which intend to discriminate the air showers of gamma-origin from those. of proton-origin by their inner structure. On the other hand the energy region of $10^{15} \sim 10^{17} \mathrm{eV}$ is important botls for particle physics and astrophysics. It is so in particle physics because this region is not covered by the existing accelerators and because there are several reports, experimental and theoretical, which point out the change of the nuclear interaction characteristics and/or existence of exotic phenomena $[1,2]$. It is so in astrophysics because there is a bend, called "knee", of the energy spectrum of the primary cosmic rays at $\sim 10^{15} \mathrm{eV}$. 


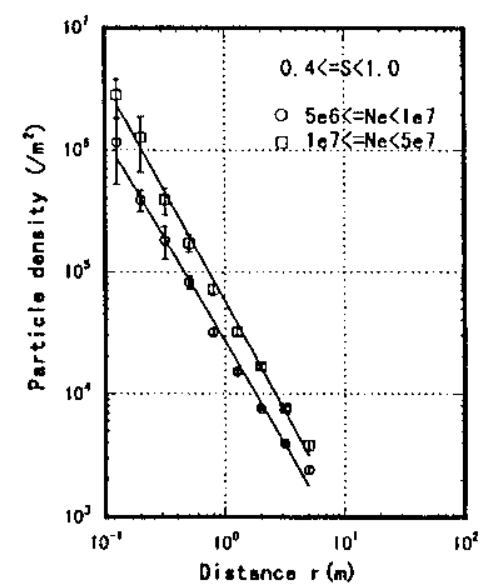

Figure 1. Lateral distribution of particle density for sir showers of $N_{e}=5 \times 10^{6} \sim 10^{7}$ and $10^{7} \sim$ $5 \times 10^{7}$. The air shower are grouped by their age parameters in respective size region.

\section{Experiment}

The experimental apparatus consist of the air shower array, the emulsion chamber and the hadron calorimeter [3]. The air slower array consists of 35 plastic scintillation detectors, which are distributed over an circular area of $50 \mathrm{~m}$ radius. The emulsion chamber and the hadron calorimeter are stored in AS.EC roon. The emulsion chamber consist of 32 units $\left(50 \times 50 \mathrm{~cm}^{2}\right.$ each $)$, each of which is $15 \mathrm{~cm} \mathrm{~Pb}$ thick and contains 14 sensitive layers of $X$-ray films. The hadron calorimeter consists of 32 units of plastic scintillator $\left(50 \times 50 \times 5 \mathrm{~cm}^{3}\right.$ each $)$, which are located beneath the emulsion chamber.

Output from each unit of the hadron calorimeter is related to the energy deposit in the scintillator, and it is converted to charged particle number using the average energy loss of a single muon in the scintillator. The number of charged particles per area of $0.25 \mathrm{~m}^{2}, n_{b}$, is called "particle density" hereafter.
The data produced by the air shower array and by the hadron calorimeter are recorded when at least one unit of the hadron calorimeter has the particle density $n_{b} \geq 10^{3}$ (particles $/ 0.25 \mathrm{~m}^{2}$ ). In this sense the mode of the run is called "hadrot calorimeter triggering". Present datia set is not. biased by the triggering mode in the region of air shower size $\mathrm{Ne}>5 \times 10^{6}$.

The lateral distribution of the particle density is determined by applying the least square fitting to the curve,

$n(r)=\frac{A}{r_{0}^{2}}\left(\frac{r}{r_{0}}\right)^{\alpha} \quad\left(r_{0}=1 \mathrm{~m}\right)$

where the parameters $A$ and $\alpha$ are to be adjusted to the experimental data.

Figure 1 shows the average lateral distribution of the particle density for the air showers of the size $N e=5 \times 10^{6} \sim 10^{7}$ and $10^{7} \sim 5 \times 10^{7}$. The avcrage density is obtained by calculating the particle densities at several distances, using parameter values $A$ and $\alpha$ of eq.(1), which are cletermined by the least square fitting in respective events.

We estimate the energy spectrum of hadrons in the air shower from the lateral distribution of the particle density, detected by the hadron calorimeter, the detail of which is described in Ref.[4]. Figure 2 shows the differential energy spectrum of hadrons in the air showers with the size $\mathrm{Ne}=5 \times 10^{\mathrm{i}} \sim 10^{7}$, together with those by the simulations. The simulations employ different models for multiple particle production; UA5 algorithm modified for hadron-nuclens collisions, VENUS[5], QGSJET[6] and HDPM[i] $]$. And a proton-dominant composition is assumed for the primary cosmic rays. The energy spectrum of hadrons by the experiment is not consistent with those by simulations in the nunber, but consistent in the power index. If one assumes a heavydominant composition of the primary cosmic rays in the simulations, the number of hadrons increases and brings larger discrepancy between the experimental and simulational datia. 


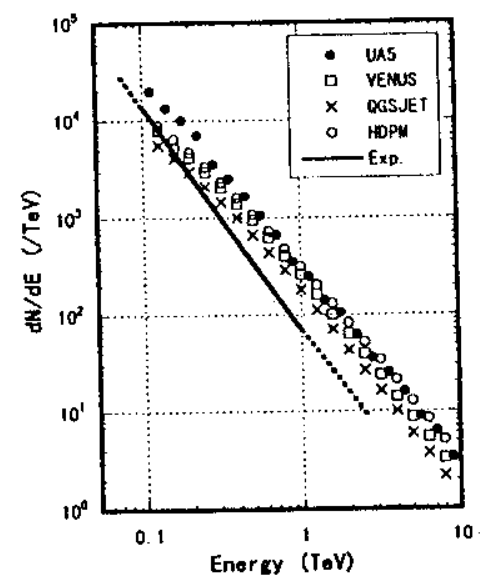

Figure 2. Differential energy spectrum of hadrons in air shower (the solid line) which is estimated from the lateral distribution of the particle density distribution. The size range of the air shower size is $\mathrm{Ne}=5^{6} \sim 10^{7}$. The predictions by various simulations are presented together. The assumptions for the primary cosmic rays are the same as the one for all the cases. The sampling of the primary energy is made for $E_{0} \geq=5 \times 10^{15} \mathrm{eV}$, and the air showers with $N_{e}=5 \times 10^{6} \sim 10^{7}$ are collected.

\section{Discussion and conclusion}

3.1. Primary cosmic-ray composition at $10^{16} \mathrm{eV}$

Figure 3(a) shows the distribution of $n_{h}$ (the diffrential number of hadrons at $E=1 \mathrm{TeV}$ ) for 62 air showers which have the size $\mathrm{Ne}=5 \times 10^{6} \sim$ $10^{\top}$. This air shower size corresponds to the primary energy of $E_{0}=10^{16} \mathrm{eV}$ on average. Figure 3(b) shows the distribution of $n_{h_{h}}$ by a simulation using UA5 algorithm for nuclear interactions. In these figures we can see;

(1) The absolute value of $m_{h}$ is different between those by the experiment and by the simulation, which is pointed out in the energy spectrum of hadrons in Fig. 2.
(2) The $n_{h}$ distribution by the experiment is wider than by simulations, probably due to the experimental errors.

(3) In the figure (b) by the simulations the proton-induced events occupy the left-hand side of the distribution and are distributed over 8 bins in the histogram.

Assuming the superposition model for the primary cosmic-ray particle of mass number $A$, we have a relation

$<\log n_{h}^{(A)}>-<\log n_{h}^{(p)}>=(1-\sigma)<\log A>$

where $\sigma$ is the index of the energy spectrum of hadrons in integral form. It means that, if we can know the average number of hadrons in the air shower $<\log n_{t}^{(p)}>$, due to the primary protons, we can obtain the average mass number of primary cosmic rays $\langle\log A>$ from the average number of harlrons in the air shower $\left\langle\log n_{h}^{(A)}\right\rangle$.
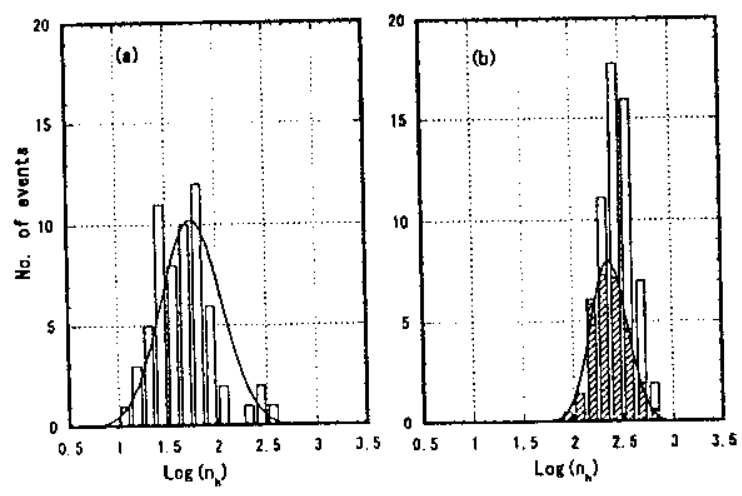

Figure 3. Distribution of $n_{t}$ (a): The experimental data of 62 events. (b) : The UA5 simulation. The munber of events is normalized to the experimental data. The latched events are protoninduced ones.

By the point (3) seen in Fig. 3(b), we assume that the $n_{f}^{(p)}$ distribution of the experimental data has a peak at the fourth bin from the 
left-hand side of the distribution. Then we have $<\log n_{h}^{(p)}>=1.44$ for the experimental data. Because we have $\left\langle\log n_{h}^{(A)}\right\rangle=1.68 \pm 0.04$ from the experimental data and $\sigma=0.8$ by the theoretical consideration, we have

$<\log A>=1.20 \pm 0.22$ or $\langle\ln A>=2.8 \pm 0.5$

One can see that the method of estimation is free from the characteristics of nuclear interaction. Figure 4 shows the average mass number of primary cosmic rays, which is estimated by the number of hadrons in air showers.

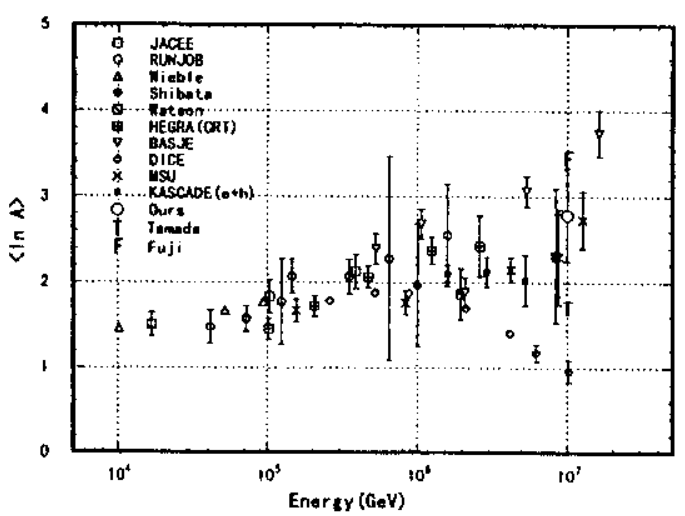

Figure 4. Average mass number of the primary cosmic rays, $\langle\ln A\rangle$, which is estimated by the number of hadrons in the air shower. The data by other experiments, compiled by KASCADE group, are shown together. The marks $\mathrm{F}$ and $\mathrm{T}$, located at $E_{0}=10^{7} \mathrm{GeV}$, are those of typical heavy-dominant[8] and proton-dominant[4] compositions.

\subsection{Nuclear interactions at $10^{16} \mathrm{eV}$}

Figure 2 shows that the number of hadrons in the air shower is lower than those by the simulations in the energy region of $10^{16} \mathrm{eV}$. This tendency is consistent with the relationship between the families and accompanied the air showers [1]. That is, the number of $\gamma$-rays in the family by the experiment is smaller than that by the simulation. It is important to point out that both data of $\gamma$-ray and hadrous are independent, because they are detected by different detertors.

Our argument is in the following way;

(1) Main assumptions in the simulations are on the hadron-air collisions and on the chemical composition of the primary cosmic rays. The experimental data indicate that at least either of the assumptions should be revised in the direction to make the energy subdivision nore rapid.

(2) Heavy-dominant hypothesis of the primary cosmic rays, proposed currently, is not effective. to remove the discrepancy.

(3) Hence we reach the conclusion that the nuclear interaction has different characteristics from those assumed in the simulations in the energy region of $10^{16} \mathrm{eV}$. That is, the multiple particle production in $10^{16} \mathrm{eV}$ is of higher multiplicity, of softer energy spectrum of the produced particles, of larger inelasticity, etc., compared with those assumed in the simulation.

\section{REFERENCES}

1. N. Kawasumi et al., Phys. Rev. D53 (1970) 3634.

2. C. M. G. Lattes et al., Phys. Rep. No.65 (1980) 151.

3. C. Aguirre et al., Nucl. Phys. B(Proc. Suppl.) 75A (1999) 186.

4. C. Aguirre et al., ICRR-Report-460-2000-4, (2000).

5. K. Werner, Phys. Rep. 232 (1993) 87.

6. N. N. Kalykov and S. S. Ostapchenko, Plys. At. Nucl. 56 (1993) 346.

7. J. N. Capdevielle, J. Phys. G 15 (1989) 909.

8. M. Anenomori, et al., Phys. Rev. D25, (1982) 2807 . 


\title{
On the penetrating showers observed in Chacaltaya two-storey
}

\author{
M. TAMada $\left({ }^{1}\right)$ \\ $\left({ }^{1}\right)$ Faculty of Science and Engineering, Kinki University, Osaka 577-8502, Japan
}

\begin{abstract}
Summary. - The penetrating nature of the cascade showers observed in the Chacaltaya two-storey chamber is compared with that of simulated $(e, \gamma)$-induced and hadron-induced cascade showers. It is shown that around $1 / 3$ of the observed penetrating showers are neither $(e, \gamma)$-induced nor hadron-induced ones. A possible explanation is given in connection with 'mini-clusters'.
\end{abstract}

PACS 94.40 - .

\section{1. - Introduction}

The Chacaltaya and Pamir emulsion chamber experiments have shown that there exist unusual phenomena which are not yet observed in the present accelerator experiments from the analysis of high energy cosmic-ray families, a bundle of electromagnetic particles and hadrons produced in the nuclear and electromagnetic cascade process in the atmosphere.[1] Those are called 'Centauro-species', multiple hadron production without association of $\pi^{0}$-mesons. It is also discussed that the nature of secondary particles is possibly different from that of ordinary hadrons in those unusual phenomena. In Ref.[2] we discussed about unusual behaviour of cascade development of the high energy showers in high-energy cosmic-ray families observed by Chacaltaya two-storey chambers no. 18 and no.19. We studied in detail how the shape of the cascade transition of the observed showers, which penetrate from the upper chamber down to the lower chamber, deviate from that of standard electromagnetic cascades expected in the uniform lead chamber. We found frequent existence of strong penetrating showers which became rejuvenated after passing through the target layer. The results were discussed in connection with 'mini-clusters', clusters which consist of extraordinarily correlated $\gamma$-rays and hadrons. $[1,3]$ Here we discuss the issue again by comparing the penetrating probability of the cascade showers with those initiated by $(e, \gamma)$ and also by hadron interaction taking into accounts the exact structure of the Chacaltaya two-storey chambers no.18 and no. 19. 


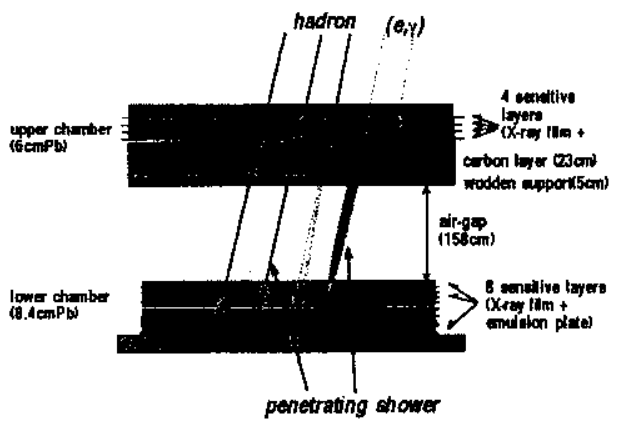

Fig. 1. - Illustration of Chacaltaya two-storey chamber no. 19 .

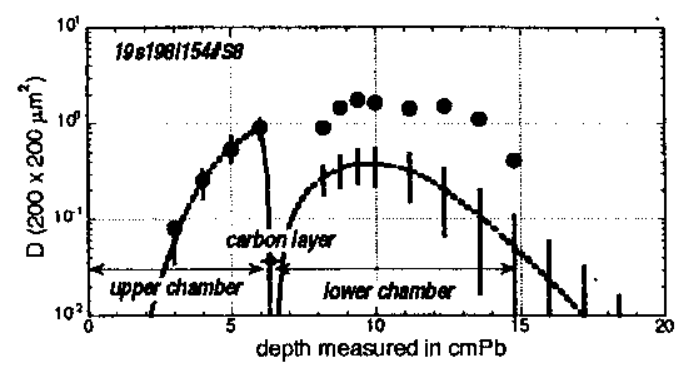

Fig. 2. - An example shower transition on spot darkness of the penetrating showers observed in Chacaltaya chamber no.19. Dotted curve is an expected shower transition best-fitted to the upper chamber data. Vertical bars are dispersion $\sigma$ of the standard transition curve.

\section{2. - Experimental data}

21. The structure of the two-storey chambers. - Fig.1 shows the basic structure of Chacaltaya two-storey chambers no.19. The chamber consists of the upper chamber of $6 \mathrm{cmPb}$, the target layer of $23 \mathrm{~cm}$ carbon (petroleum pitch), wooden support of $5 \mathrm{~cm}$ thick, the air gap of $158 \mathrm{~cm}$ height and the lower chamber of $8.4 \mathrm{cmPb}$. Four sensitive layers (X-ray film and nuclear emulsion plate) are inserted in the upper chamber and eight sensitive layers in the lower chamber. In the chamber no.18, the thickness of the upper chamber is $7 \mathrm{cmPb}$ and 5 sensitive layers (composed of only X-ray films) are inserted. The other structure of the chamber no.18 is just same to that of the chamber no.19. Showers detected in the upper chamber are mainly $(e, \gamma)$-induced ones with small admixture of hadron-induced ones. Showers detected in the lower chamber, on the other hand, are those initiated by nuclear interactions in the target layer (C-jets) and in the lead plates of the lower chamber itself $(\mathrm{Pb}$-jet-lower).

22. Selection of the events. - In the present analysis we pick up showers in the high energy cosmic-ray families, observed by the Chacaltaya chamber no.18 and no.19, which satisfy the following conditions;

1) the total visible energy is grater than $100 \mathrm{TeV}$ and

2) the event has at least two penetrating showers which are observed both in the upper chamber and in the lower chamber.

The latter condition is necessary to confirm the exact upper-lower correspondence.

\section{3. - Penetrating cascade showers}

Some of the showers observed in the upper chamber can be followed down into the lower chamber. We define it as a penetrating shower when its spot darkness, $D$, measured by a $200 \times 200 \mu \mathrm{m}^{2}$ slit, is larger than 0.2 in at least two layers in the upper chamber and at least one layer in the lower chamber. A typical example of the shower transition of the penetrating shower is shown in Fig.2. We summarise in Table 1 the number of showers with visible energy $E(\gamma) \geq 10 \mathrm{TeV}$ observed in the upper chamber, those which penetrate into the lower chamber among them and those observed only in the lower chamber (C-jets, Pb-jets-lower). 
TABLE I. - Number of high energy showers, $E(\gamma) \geq 10 \mathrm{TeV}$, observed in the chambers no.19 and no. 18 .

\begin{tabular}{lccc}
\hline & \multicolumn{2}{c}{ chamber no.19 } & chamber no.18 \\
& Japanese part & all & all \\
\hline $\begin{array}{l}\text { atmospheric families of } \Sigma E(\gamma) \geq 100 \mathrm{TeV} \\
\text { with at least two penetrating showers }\end{array}$ & 15 events & 32 events & 14 events \\
\hline
\end{tabular}

(a) no. of showers observed in the upper chamber

\begin{tabular}{lccc}
\hline (b) no. penetrating showers among (a) & $47(61)$ & $83^{\dagger}(108)$ & $31(45)$ \\
\hline $\begin{array}{l}\text { (c) no. of showers of observed only in the } \\
\text { lower chamber (C-jets, Pb-jets-lower) }\end{array}$ & 7 & 24 & 18 \\
\hline $\begin{array}{l}\text { (d) expected no. of penetrating showers of } \\
\text { hadronic origin }\end{array}$ & $2.7 \pm 1.0$ & $9.1 \pm 1.9$ & $7.5 \pm 1.8$ \\
\hline $\begin{array}{l}\text { (e) no. of }(e, \gamma) \text {-induced showers in the up- } \\
\text { per chamber }\end{array}$ & $107 \pm 10$ & $179 \pm 14$ & $104 \pm 10$ \\
\hline $\begin{array}{l}\text { (f) expected no. of penetrating }(e, \gamma)- \\
\text { induced showers [(a)-(d)] }\end{array}$ & $26 \pm 2.5$ & $44 \pm 3.2$ & $15 \pm 1.5$ \\
\hline $\begin{array}{l}\text { (g) excess of penetrating showers over ex- } \\
\text { pectation [(b)-(d)-(f)] }\end{array}$ & $18.3 \pm 7.4$ & $30.1 \pm 9.8$ & $8.5 \pm 6.0$ \\
\hline
\end{tabular}

Figures in the parenthesis in the raw (b) is all the number of measured penetrating showers where detection threshold darkness is $D_{t h} \sim 0.1$.

f) In the Chacaltaya chamber no.19, full data of spot darkness of the penetrating showers are available at present for a half of the chamber which are measured in Japan. Hence the number of penetrating showers in all events in chamber no.19 which satisfy the present definition is estimated from the number of all the measured penetrating showers by multiplying a factor $47 / 61$, obtained in the Japanese part data.

\section{4. - Simulations of cascade showers in the two-storey chambers}

41. Hadron-induced showers. - We use two different models for hadron-nucleus interactions, one is QGSJET [4] based on the Gribov-Regge theory of multiple Pomeron exchanges and the other is phenomenological UA5 algorithm [5] modified for hadronnucleus interaction using a geometrical approach. In the nuclear cascade all hadrons, produced in the collisions during passage through the chamber, are followed until their energy falls below $80 \mathrm{GeV}$ or they leave the chamber. The interaction mean free path of hadron-nucleus interactions is assumed to decrease with increasing interaction energy, e.g., $\Lambda^{p-P b}(E)=15.9 \mathrm{cmPb}, \Lambda^{\pi-P b}(E)=17.5 \mathrm{cmPb}, \Lambda^{p-C}(E)=62.4 \mathrm{cmC}$ and $\Lambda^{\pi-C}(E)=79.4 \mathrm{cmC}$ at $E=10^{14} \mathrm{eV}$.

For $\gamma$-rays of $E_{\gamma} \geq 1 \mathrm{GeV}$, which are mainly decay products of $\pi^{0}$ 's produced in the collisions, we further calculate the three-dimensional electromagnetic cascade development in the chamber using the Monte-Carlo code formulated by M. Okamoto and T. Shibata [6], in which the LPM effect is also taken into accounts. Electrons and photons are followed until their energies fall below $1 \mathrm{MeV}$. The electron number density, $\rho_{e}$, is converted to the local spot darkness, $d$, of $\mathrm{X}$-ray film, by using the characteristic 
TABLE II. - Number of hadron-induced showers $\left(E_{h}(\gamma) \geq 10 \mathrm{TeV}\right)$.

\begin{tabular}{lcccccc}
\hline & \multicolumn{2}{c}{ chamber no.19 } & \multicolumn{2}{c}{ chamber no.18 } \\
\hline & QGSJET & \multicolumn{2}{c}{ modified UA5 } & \multicolumn{2}{c}{ QGSJET } \\
\hline incident & pion & proton & pion & proton & pion & proton \\
\hline (a) penetrating & 187 & 195 & 199 & 254 & 228 & 224 \\
\hline (b) visible only in the lower chamber & 609 & 496 & 530 & 546 & 572 & 507 \\
\hline ratio $=(\mathrm{a}) /(\mathrm{b})$ & 0.31 & 0.39 & 0.38 & 0.46 & 0.39 & 0.44 \\
\hline
\end{tabular}

In each set of the calculations, 2,000 particles are sampled from the spectra described in $\S 4.1$.

relation for the $\mathrm{N}$-type $\mathrm{X}$-ray film, and finally we obtain the transition curve of the spot darkness $D$, measured by a $200 \times 200 \mu \mathrm{m}^{2}$ slit, vs. depth $T$ throughout the chamber. The experimental error of the measurement of spot darkness $D$ is also taken into accounts by adding noise $\Delta D$ in each spot darkness where $\Delta D$ is sampled from Gaussian distribution with $\sigma_{D}=0.1 D$. Protons and pions of $E_{h} \geq 20 \mathrm{TeV}$ are sampled from the energy spectrum $I\left(\geq E_{h}\right) \propto E_{h}^{-1.2}$ and from zenith angular distribution $I(\leq \cos \theta) \propto(\cos \theta)^{-17}$. $\left({ }^{1}\right)$

42. $(e, \gamma)$-induced showers. - We also calculate electromagnetic cascade development in the chamber initiated by $\gamma$-rays and electrons using the above mentioned Monte-Carlo code assuming the energy spectrum and zenith angle distribution of the $(e, \gamma)$ arriving at the chamber as $I\left(\geq E_{\gamma}\right) \propto E_{\gamma}^{-2}$ and $I(\leq \cos \theta) \propto(\cos \theta)^{-17}$ respectively.

\section{5. - Penetrating showers}

Some of the hadrons interact with $\mathrm{Pb}$-nucleus in the upper chamber and those hadroninduced showers can be visible in the upper chamber. According to the simulations, almost all hadron-induced showers in the upper chamber penetrate into the lower chamber. The number ratio of those penetrating hadron-induced showers to the showers visible only in the lower chamber ( $\mathrm{C}$-jets and $\mathrm{Pb}$-jets-lower) is given in Table 2. As is seen in the table, the ratio is around 0.38 in the chamber no.19 and 0.42 in the chamber no. 18 on the average. Then we can obtain the expected number of hadron-induced penetrating showers ( (d) of Table 1) from the number of showers which are observed only in the lower chamber in the experiment ( (c) of the Table 1). The number of $(e, \gamma)$-induced showers in the upper chamber is then given by subtracting the above number of hadron-induced penetrating showers from the number of showers in the upper chamber and the result is shown in (e) in the Table. The penetrating probability of $(e, \gamma)$ - induced showers of $E_{e, \gamma} \geq 10 \mathrm{TeV}$ is summarized in Table.3. According to the simulations of the atmospheric

(1) The attenuation length of the atmospheric families is usually considered to be $\lambda_{\text {att }} \sim 90$ $\mathrm{g} / \mathrm{cm}^{2}$. Then the zenith angle distribution of those is given by $I(\leq \cos \theta) \propto(\cos \theta)^{-8}$ at Chacaltaya. Due to the selection condition that the events have at least two penetrating showers, most of the selected events have small zenith angle and the zenith angle distributuion of those becomes very steep. 
TABLE III. - Penetrating probability of $\gamma$-ray and electron-induced showers $\left(E_{\gamma, e} \geq 10 \mathrm{TeV}\right)$.

\begin{tabular}{lccc}
\hline & $\gamma$-ray & electron & $(e, \gamma)$ \\
\hline chamber no.19 & 0.26 & 0.23 & 0.245 \\
\hline chamber no.18 & 0.17 & 0.12 & 0.145 \\
\hline
\end{tabular}

In each set of the calculations, 2,000 particles are sampled from the spectra described in $\$ 4.2$. 'The figure in the last column is obtained assuming the number of $\gamma$-rays is two times more than that of $e^{ \pm}$in the atmospheric families.

farnilies using CORSIKA/QGSJET code, the number ratio of $\gamma$-rays to $e^{ \pm}$in the families of $\Sigma E(\gamma) \geq 100 \mathrm{TeV}$ is found to be 2 to 1 . Then the average penetrating probability of $(e, \gamma)$-induced showers is 0.245 for the chamber no.19 and 0.145 for the chamber no. 18 . The expected number of penetrating showers of $(e, \gamma)$ origin is calculated using those penetrating probabilities and is shown in (f) of Table 1 , around one half of the observed penetrating showers can be considered to be $(e, \gamma)$ origin. 'The excess of the number of penetrating showers over the expectation ( shown in (g) Table 1) amounts to $\sim 37 \%$ of all the penetrating showers in the chamber no.19 and $\sim 27 \%$ in the chamber no.18, though the statistical error is rather large.

\section{6. - Discussions}

We have shown that around $37 \%(27 \%)$ of penetrating showers, $\sim 16 \%(\sim 8 \%)$ of all the showers in the upper chamber, observed in the Chacaltaya two-storey chamber no.19 (no.18) are neither $(e, \gamma)$-induced nor hadron-induced showers. One of the possible explanations is to assurne an existence of extremely collimated pair of a $\gamma$-ray and a hadron. That is, if the mutual distance between a $\gamma$-ray and a hadron is extremely small, e.g., less than $\sim 1 \mathrm{~mm}$, and the $\gamma$-ray-induced showers is observed in the upper chamber and the hadron-induced shower is observed in the lower chamber, we would possibly misidentify those two as a penetrating shower. If a $\gamma$-ray make electromagnetic interactions in the atmosphere, we can observe collimated several $(e, \gamma)$-particles and a hadron as a 'mini-cluster' which are often found in the exotic events. Possible existence of hadron-bundles in which the mutual distance of the constituent hadrons is extremely small is also discussed in the analysis of shower transition of high-encrgy hadronic showers observed in the Pamir thick lead chamber[7, 8].

\section{REFERENCES}

[1] Chacaltaya and Pamir Collaboration (L.T.Baradzei et al.); Nucl. Phys. B 370 (1992) 365

[2] Y.Funayarna \& M.Tamada: J. Phys. Soc. Japan 55, (1986) 2977

[3] M.Tamada \& Y.Funayama: J. Phys. Soc. Japan 55, (1986) 2996

[4] N.N.Kalmykov, S.S.Ostapchenko \& A.I.Pavlov: Bull. Russ. Acad. Sci. (physics) 58 (1994)

[5] UA5 Collaboration (G.L.Ainer et al.): Nucl. Phys. B 291 (1987) 445

[6] M.Okamoto \& T.Shibata: Nucl. Instr. Meth. A257 (1987) 155

[7] M.Tamada \& A.Ohsawa: Nucl. Phys. B 581 (2000) 73

[8] M.Tamada \& V.V.Kopenkin: Nucl. Phys. B 494 (1997) 3 


\title{
Study of Nuclear Interactions by Observing Family and Air Showers at Mt. Chacaltaya
}

\author{
C. Aguirre $\left({ }^{1}\right)$, H. Aoki $\left({ }^{2}\right)$, K. Hashimoto $\left({ }^{3}\right)$, K. Honda $\left({ }^{4}\right)$, N. Inove $\left({ }^{5}\right)$, \\ N. Kawasumi $\left({ }^{3}\right)$, Y. Maeda $\left({ }^{6}\right)$, N. Martinic $\left({ }^{1}\right)$, T. Matano $\left({ }^{7}\right)$, N. Ohmori $\left({ }^{6}\right)$, \\ A. OHSAWA $\left({ }^{8}\right)$, K. Shinozaki $\left({ }^{5}\right)$, M. Tamada $\left({ }^{9}\right)$, R. Ticona $\left({ }^{1}\right)$, and I. Tsushima $\left({ }^{3}\right)$ \\ (') Instituto de Investigaciones Fisicas, Universidad Mayor de San Andres, La Paz, Bolivia. \\ $\left({ }^{2}\right)$ Faculty of Engineering, Soka University, Hachioji, Tokyo, 192-8577 Japan. \\ ( $\left.{ }^{3}\right)$ Faculty of Education, Yamanashi University, Kofu, Yamanashi, 400-8501 Japan. \\ (4) Faculty of Engineering, Yamanashi University, Kofu, Yamanashi, 400-8511 Japan. \\ (5) Faculty of Science, Saitama University, Urawa, Saitama, 388-8570 Japan. \\ ( $\left.{ }^{6}\right)$ Faculty of Science, Kochi University, Kochi, 780-8520 Japan. \\ (7) Shibakubo 3 - 28-10, Tanashi, Tokyo, 188 Japan. \\ $\left(^{8}\right)$ Institute for Cosmic Ray Research, University of Tokyo, Kashiwa, Chiba, 277-8582 Japan. \\ $\left({ }^{9}\right)$ Faculty of Sci. and Eng., Kinki University, Higashi-Osaka, Osaka, 577-8582 Japan.
}

\section{1.- - Introduction}

Study of nuclear interactions by two-storey emulsion chambers, which consist of upper and lower emulsion chambers and a jet-producing layer in between, made clear characteristics of multiple particle production in the energy region of $10^{14} \mathrm{eV}[1]$, most of which are confirmed by the experiments at CERN $\ddot{p} p$ collider. [2] This owes to excellent performance of emulsion chamber in energy and position determination. Next step of the study in $10^{15} \sim 10^{17} \mathrm{eV}$ is made by observing nuclear interactions in the atmosphere, called "families", by simple emulsion chambers of large area, because the intensity of high energy cosmic rays is very small.

The farnily data differ in several points from those of target interactions which are obtained by two-storey chambers. Those are (1) unknown interaction height, (2) pollution of the event by overlapping of successive nuclear interactions and by secondary interactions of the produced particles, and (3) bias of the observed events due to high detection threshold energy of the emulsion chamber. Consequently the data of families are used only for the purpose to support the discoveries, made by two-storey chambers, in high energy region.[1]

To overcome such shortcomings we started simultaneous observation of families and air showers which accompany the families. $[3,4]$ That is, we operate an emulsion chamber, a hadron calorimeter and an air shower array simultaneously. Importance of the experiment is to bridge the families and air showers, because both experiments have accumulated large amount of data independently. Therefore a large scale of the experiment is not needed. 


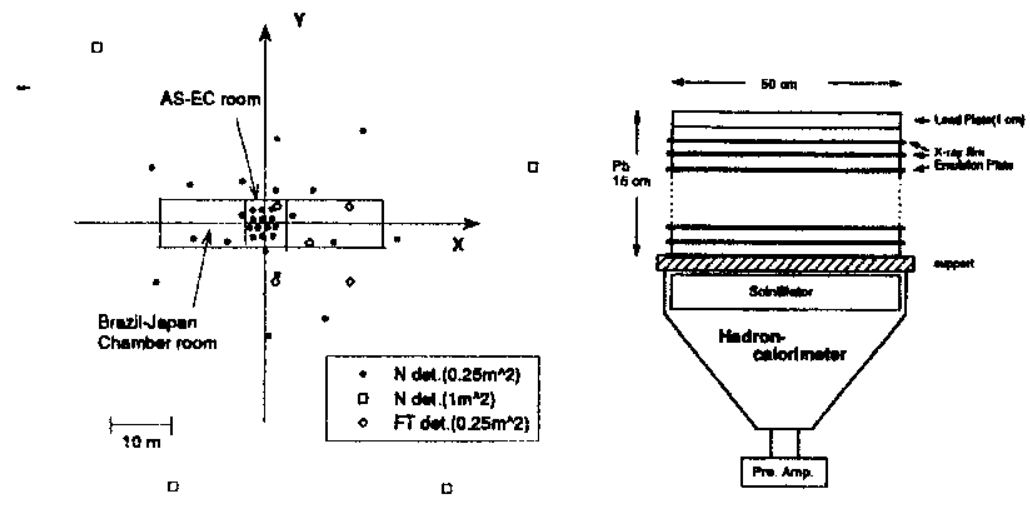

Fig. 1. - Air shower array (left) and a unit of emulsion chamber and hadron calorimeter (right). Emulsion chamber and hadron calorimeter, consisting of 32 units $(50 \mathrm{~cm} \times 50 \mathrm{~cm}$ each), are stored in AS-EC room at the center of the air shower array.

\section{2. - Experimental set-up}

21. Detectors. - The experiment is carried out at Mt. Chacaltaya $(5,200 \mathrm{~m}$, Bolivia) The following is a brief description of the detectors and of their performance.

(1) Air shower array

The air shower array consists of 35 plastic scintillators, which are distributed over an circular area of $50 \mathrm{~m}$ radius. (Fig. 1.) The available data are of arriving time, direction, center position, size, etc. of air showers.

(2) Emulsion chamber

The emulsion chamber, which is located in the center of the air shower array, consists of 32 units $(50 \mathrm{~cm} \times 50 \mathrm{~cm}$ each). Each unit is $30 \mathrm{~cm} \mathrm{~Pb}$ thick with 14 sensitive layers of $\mathrm{X}$-ray films and/or nuclear emulsion plates. (Fig. 1)

An electron or a photon of high energy, incident upon the chamber, produces a cascade shower in the chamber, and the electron component in the shower is detected by several successive sensitive layers. In this way the emulsion chamber is sensitive to electrons and photons, called " $\gamma$-rays" collectively, incident upon the chamber, and determines their positions and energies. $\left({ }^{1}\right)$ Emulsion chamber has high detection threshold energy of $E_{t h}=1 \sim 3 \mathrm{TeV}$.

A family, a nuclear collision in the atmosphere which is observed by emulsion chamber, is a bundle of showers with parallel direction of incidence. Available data are of direction, center position, total observed energy, etc. of the families, but not of the arriving time because the development of sensitive materials is made after $1 \sim 2$ years of exposure.

(3) Hadron calorimeter

The hadron calorimeter of 32 plastic scintillators $(50 \mathrm{~cm} \times 50 \mathrm{~cm}$ each) is located beneath the emulsion chamber. (Fig. 1.) Each unit of them detects charged particles under the emulsion chamber and hence 32 units of them supply us with a two-dimensional map of charged particle density over the hadron calorimeter. These charged particles,

(1) Emulsion chamber is sensitive to hadrons, incident upon the chamber, too, because a hadron produces a bundle of $\gamma$-rays in the chamber through the nuclear collision with $\mathrm{Pb}$. 
mainly electrons and hadrons, are produced by hadrons, incident upon the emulsion chamber, through nuclear and electromagnetic cascade processes in lead of the emulsion chamber. It is worth noting that cascade showers which are produced by electrons and photons, incident upon the emulsion chamber, are absorbed completely before arriving at the bottom of the chamber. The available data are of center position, arriving time, size, etc. of the hadron component, estimated from the charged particle density distribution, in the air shower.

22. Corresponding air showers to families. - Families, which have no data of arriving time, are correlated with the events of hadron calorimeter by the coincidence of the center of the event, and events of the hadron calorimeter is correlated with air showers by the
coincidence of their arriving time.

\section{3. - Analysis of experimental data}

31. Assumptions in the simulation. - Analysis is made by comparing experimental data with those of simulated events, because nuclear interactions are not observed directly in most of the families. Simulation of air shower events is made on the following
assumptions.[3]

(i) Primary cosmic rays

Energy and atomic number of primary cosmic rays are sampled from the energy distribution of

$$
E^{-\gamma-1} d E \quad(\gamma=1.7)
$$

and from so-called normal composition of Table 1[5], respectively. The assumed composition is obtained by extrapolating the one in low energy region where direct measurement
is possible.

TABLE I. - Composition of the primary cosmic rays, assumed in the simulation

\begin{tabular}{c|ccccc}
\hline$E_{0}(\mathrm{eV})$ & $\mathrm{H}(\%)$ & He $(\%)$ & CNO $(\%)$ & Heavy (\%) & Fe (\%) \\
\hline $10^{15}$ & 42 & 17 & 14 & 14 & 13 \\
$10^{16}$ & 42 & 13 & 14 & 15 & 16 \\
\hline
\end{tabular}

(ii) Hadron-air collisions

(1) Collision mean free path of hadrons in the air

$$
\begin{array}{ll}
\lambda_{\text {air }}=760 \sigma_{\text {inel }}^{-0.63} \quad\left(\mathrm{~g} / \mathrm{cm}^{2}\right) & \\
\sigma_{\text {inel }}=\sigma_{0}\left[1+0.0273 \epsilon+0.01 \epsilon^{2} \theta(\epsilon)\right] & (\mathrm{mb})
\end{array}
$$

where $\epsilon=\ln \left(E_{0} / 200 \mathrm{GeV}\right)$ and $\theta(x)$ is a step function. The constant $\sigma_{0}$ is $32.2(\mathrm{mb})$

and $20.3(\mathrm{mb})$ for nucleon and pion collisions, respectively.

(2) The collision of a hadron with an air nucleus follows the geometrical model.

(3) Energy distribution of produced particles in inelastic collisions.

In each collision of a hadron with nucleons in an air nucleus, the multiple particle production is described by UA5 algorithm[6], which is a phenomenological simulation code to describe the UA5 Collaboration data by CERN $\bar{p} p$ collider.[7] It is worthy noting that the Feynman scaling law is violated both in the central and forward regions in the $x$-distribution of UA5 algorithm.[4]

32. Total observed energy in the family. - We obtain the total observed energy in the family by summing up the shower energies in the family, which is denoted by $\sum E_{\gamma}$. 
Fig. 2 presents the avcrage total observed energy in the family for several intervals of air shower size $N_{e}$.[3] The selected events are air showers which are accompanied by families with $n_{\gamma} \geq 5$ and $\sum E_{\gamma} \geq 10 \mathrm{TeV}\left(E_{t h} \geq 2 \mathrm{TeV}\right)$. The air shower size is a good measure relation $E_{0} / N_{e} \sim 2$ of primary particle which initiates the air shower. $\left({ }^{2}\right)$ That is, the and primary composition is shown to be almost independent of the nuclear interactions at Mt. Chacaltaya.
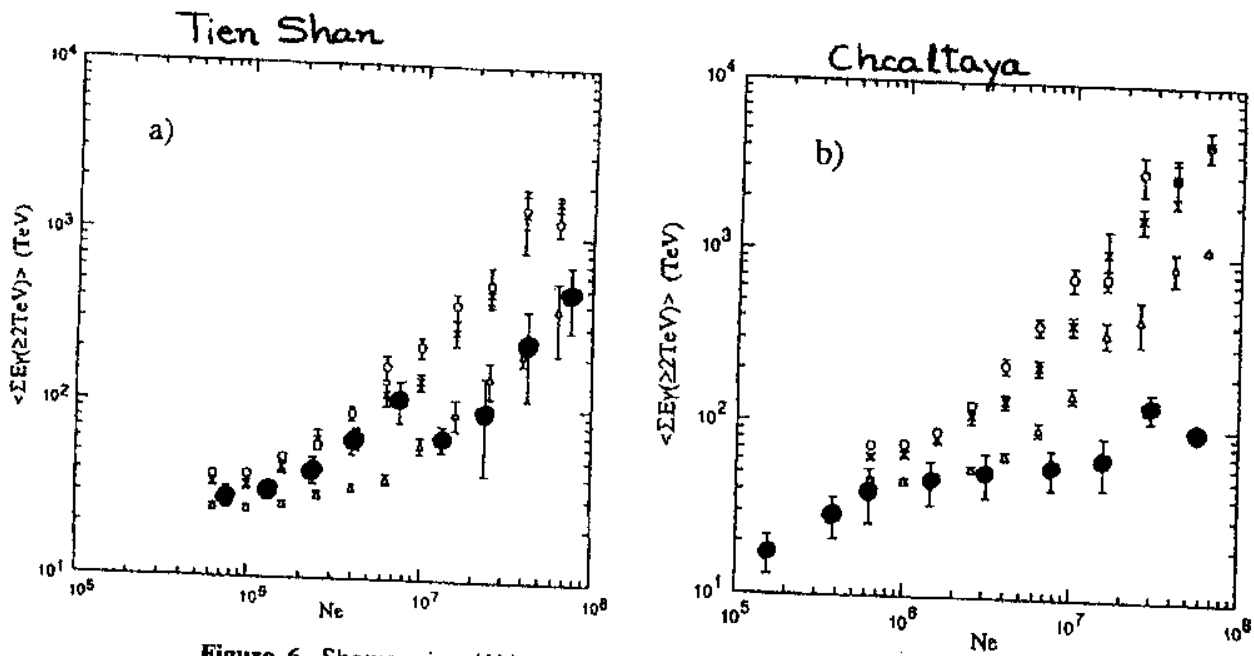

Figure 6. Shower-size $\left(N_{c}\right)$ dependence of average family energy, $\left(\sum E_{\gamma}\right\rangle:\langle a)$ for the HADRON experiment at Tien-Shan, and (b) for the sys experiment at Chacaltaya. 'heavy-dominat' $(O)$ for model-A with 'normal' chemical composition, $(X)$ model-A with composition and ( $\mathcal{L}$ experimentaldion, and $(\Delta)$ model-B with 'normal' chemical

Fig. 2. - Correlation between the total observed energy in the family and air shower size, obtained by the experiments at Tien Shan (a) and at Mt. Chacaltaya (b). Model-A stands for
UA5 algorithm.

The figure shows that the total observed energy in the family is lower than that by the simulation in large size region of $N_{e} \geq 5 \times 10^{6}$. Similar type of experiment at Tien Shan $(3,300 \mathrm{~m}$, Kazakhstan) obtained the same results, which is presented in Fig. 2.[8] They indicate that the subdivision of energy in the atmosphere is stronger at high energies of $\sim 10^{16} \mathrm{eV}$ than the one assumed in the simulation. That is, at least either of the assumptions in the simulation, the energy distribution of produced particles or the primary composition, should be modified into the one of stronger energy subdivision. Consequently following hypotheses are possible. (1) The Feynman scaling law is violated in the forward region more strongly than assumed
in UA5 algorithm.

(2) The composition of the primary cosmic rays becomes heavier or the fraction of irons becomes larger than the one in low energy region.

$\left({ }^{2}\right)$ Air shower size is defined as the number of charged particles in the air shower. It consists of hadron, electron $\left(e^{+}\right.$and $\left.e^{-}\right)$and muon components, among which electron component is 
Fig. 2 shows also that a proposed heavy composition of the primary cosmic rays[9] reduces but does not describe fully the observed discrepancy. Therefore we reach a conclusion that the proposal (1) is valid at high energies.

3. 3adron component in the air shower. - We can estimate the energy-lateral distribution of hadrons, incident upon the emulsion chamber, from the charged particle density map which is obtained by the hadron calorimeter, by taking into account the nuclear and electromagnetic cascade processes in lead of the chamber.[4]

Fig. 3 presents the expected differential energy spectrum of hadrons in the air shower. The energy spectrum is expressed in the differential form, because the observed lateral distribution of charged particle density is limited in the region of $r=0 \sim$ several meters. The figure shows that the number of hadrons is smaller than that by the simulation, indication of which is consistent with the one in Fig. 2. It is important that both components, the high energy $\gamma$-rays of $\geq 2 \mathrm{TeV}$ and the hadrons of $0.1 \sim 1 \mathrm{TeV}$, in the air shower show the same tendency.

KASCADE expcriment (at sea level, Germany) obtained the same conclusion from the study of hadronic cores of extensive air showers by the large hadron calorimeter. $[10]$

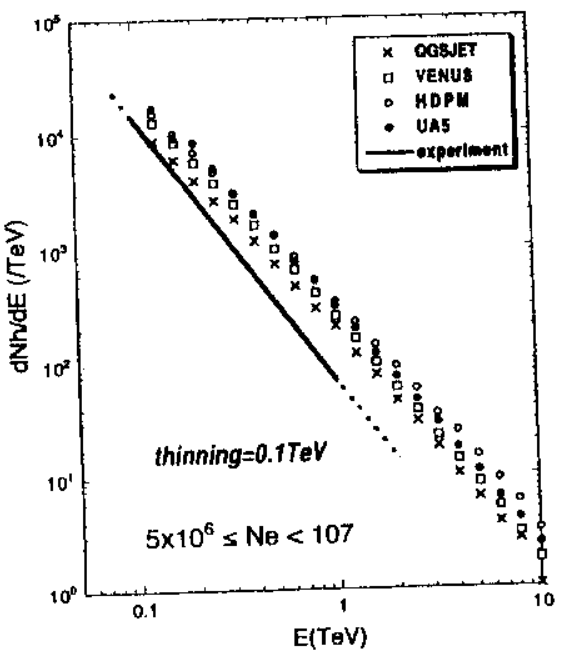

Fig. 3. - Differential energy spectrum of hadrons in the air showers of the size region $N_{e}=5 \times 10^{6} \sim 10^{7}$. The solid line part corresponds to the experimental data, because the observed lateral distribution of charged particle density is limited in the region of $r=0 \sim$ several meters. Plots are those by simulation codes which are employed presently to follow atmospheric diffusion of cosmic rays.

\section{REFERENCES}

[1] J.A. Chinellato et al., Prog. Theor. Phys. Suppl. No.76 (1983) 1.

[2] F. Halzen and N. Yaindagni, Nucl. Phys. B190 (1987)213.

[3] N. Kawasumi et al., Phys. Rev. D53 (1996) 3534.

[4] C. Aguirre et al., Phys. Rev. D62 (2000) 032003.

[5] S.I. Nikolsky, Proc. 3rd Sympo. on Cosmic Rays and Particle Physics, Tokyo (1984) (Inst. for Cosmic Ray Research, Univ. of Tokyo), 507.

[6] G.J. Alner et al. (UA5 Collaboration), CERN-EP/86-213 (1986).

(7) G.L. Alner et al. (UA5 Collaboration), Z. Phys. C33 (1986) 1; Nucl. Phys. B291 (1987) 445; Phys. Rep. Nos.5 and 6 (1987) 247.

[8] V.V. Arabkin, S.I. Nikolsky, K.V. Cherdyntseva and S.B. Schaulov, FIAN Preprint No.140 (1990) (Lebedev Physical Institute) (in Russian); Proc. 22nd Intern. Cosmic Ray Conf. (Dublin) (1991) Vol.4 pp.141, 269, 273.

[9] J.R. Ren et al., Phys. Rev. D38 (1988)1404.

[10] T. Antoni et al., J. Phys. G 25 (1999) 2161. 


\title{
Nuclear Interactions and Highest Energy Air Showers
}

\author{
A. OhSawa $\left({ }^{1}\right)$, E.H. ShibuYa $\left({ }^{2}\right)$, and M. Tamada $\left({ }^{3}\right)$ \\ (1) Institute for Cosmic Ray Research, University of Tokyo, Kashiwa, Chiba, 277-8582 Japan. \\ $\left({ }^{2}\right)$ Inst. de Fisica, Univ. Estadual de Campinas, 13083-970 Campinas, São Paulo, Brasil. \\ (3) Faculty of Sci. and Eng., Kinki University, Higashi-Osaka, Osaka, 577-8582 Japan.
}

\section{1. - Introduction}

At present the energy spectrum of the primary cosmic rays, which is obtained by observing the highest energy air showers, looks to extend beyond the GZK cut-off energy. And there are several experimental efforts to confirm this discovery and many ambitious proposals to describe it, because it is one of the most interesting puzzles to be solved. One should however keep in mind that high energy nuclear interactions, which we cannot say to be established well above $10^{16} \mathrm{eV}$, are assumed to obtain the energy spectrum from the observed data of air shower size.

In this report we discuss whether the energy distribution of produced particles in multiple particle production, formulated by us[1], describes the highest energy $\left(\geq 10^{18} \mathrm{eV}\right)$ air showers or not. The formulation is made phenomenologically on the basis of experimental data of direct observation by accelerator and cosmic-ray experiments, assuming that the Feynman scaling law is valid at low energies but is violated at high energies. It is a merit of discussing the highest energy air showers that the energy dependence of the nuclear interaction characteristics shows itself in the most distinct way. Discussion enables us to examine how the nuclear interactions affect the air shower size at the highest energy region of $10^{18} \sim 10^{20} \mathrm{eV}$, too.

\section{2. - Air Showers}

\section{2'1. Elementary processes for air showers.-}

(1) Inelastic collision mean free path of hadrons in the air.

$$
\lambda_{N}\left(E_{0}\right)=\lambda_{N}\left(E_{0} / B\right)^{-\beta} \quad \lambda_{\pi}\left(E_{0}\right)=(1 / \xi) \lambda_{N}\left(E_{0}\right)
$$

where $\beta=0.056, B=10^{3} \mathrm{GeV}$ and $\xi \equiv \sigma_{\pi N} / \sigma_{N N}=0.71$.

(2) Energy distribution of the surviving particle

$$
(1-b) \delta\left(E-(1-K) E_{0}\right) d E
$$

where $K$ is the total inelasticity to be discussed below. The charge exchange probability of the surviving pion $b$ is 0 and 0.3 for nucleon and pion collisions, respectively. That is, the charge exchange of the surviving pion, i.e. $\pi^{ \pm} \rightarrow \pi^{0}$, is an important process to be taken into account, because the inelasticity is 1.0 in the process.

(3) Energy distribution of charged produced particles

$$
\varphi\left(E_{0}, E\right) d E=a D\left(1-a^{\prime} x\right)^{d} / x d x \quad\left(x \equiv E / E_{0}, a=\left(E_{0} / A\right)^{\alpha}, a^{\prime}=\left(E_{0} / A\right)^{\alpha^{\prime}}\right)
$$

where $D=2(d+1) / 3, d=4.0, A=200 \mathrm{GeV}, \alpha=0.105$ and $\alpha^{\prime}=0.210$. The formula is obtained on the basis of experimental data of direct observation by accelerator experiments $(\sqrt{s}=53,200,546,630$ and $900 \mathrm{GeV}$ of CERN SPS $\bar{p} p$ collider $)[2,3,4]$ 
TABLE I. - Scaling violation parameters assumed in the models

\begin{tabular}{lcccc} 
& $\alpha$ & $\alpha^{\prime}$ & Feynman scaling law & Remark \\
\hline Model-0 & 0 & 0 & valid & $<K>=0.5$ \\
Model-1 & 0.105 & 0.105 & violated & $<K>=0.5$ \\
Model-2 & 0.105 & 0.210 & violated & the best-fit to the data \\
\hline
\end{tabular}

and by cosmic-ray experiment $(<\sqrt{s}>=500 \mathrm{GeV}$ of emulsion chamber experiment at Mt. Chacaltaya) [5]. [1]

The formula with $a=a^{\prime}=1.0$, which is attained at low energy of $E_{0} \sim 200 \mathrm{GeV}$, is one of the empirical distributions to follow the Feynman scaling law.[6] Validity of the law is verified experimentally in the energy region $\sqrt{s} \leq 63 \mathrm{GeV}$.[7] One can see in the formula that the law is violated strongly at high energies and consequently the average inelasticity decreases appreciably at high energies. (See Fig. 1.)

The formula leads to the energy dependences of charged multiplicity and average total inelasticity (See Fig. 1.), defined as \[ m\left(E_{0}\right) \equiv \int \varphi\left(E_{0}, E\right) d E<<K>\equiv \frac{3}{2} \int \varphi\left(E_{0}, E\right) E d E \]
where the factor $3 / 2$ is due to charge independence of produced pions on the assumption that all the produced particles are pions.

(4) Inelasticity is assumed to be distributed uniformly between 0 and $2\langle K\rangle$.

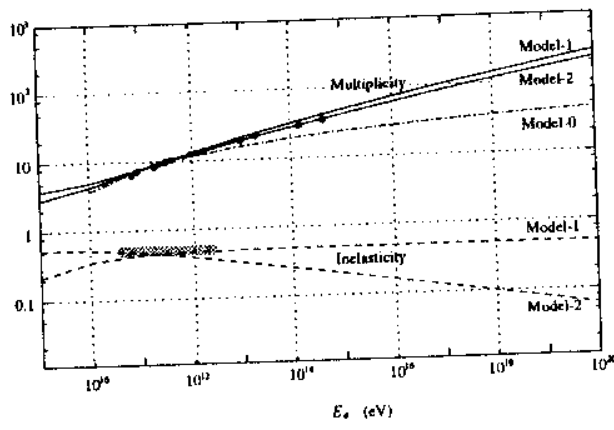

Fig. 1. - Energy dependences of charged multiplicity and average total inelasticity. Model-0, Model-1 and Model-2 are explained in Table 1 . The hatched area is the energy region where the Feynman scaling law is verified experimentally. Full circles are the experimental data of charged multiplicity.

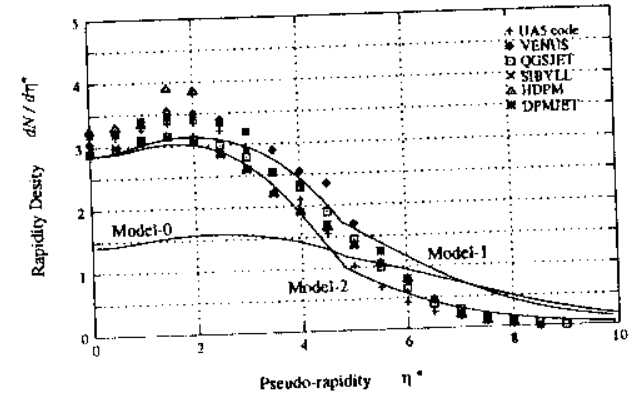

Fig. 2. - The pseudo-rapidity density distributions at $\sqrt{s}=546 \mathrm{GeV}$, assumed in Table 1 (solid lines) and obtained by the simulation codes (plots) which are used recently to simulate the diffusion of cosmic rays in the atmosphere.

22. Comparison with the models used in simulations. - We assume three types of energy distributions for the discussion made below. (See Table 1.)

It is interesting to see whether the formula of eq.(1) is consistent with the nuclear interaction models, such as VENUS, QGSJET, DPMJET, SYBILL, HDPM and UA5 code, [8] which are incorporated in the simulations to follow the atmospheric diffusion of cosmic rays. Fig. 2 shows the pseudo-rapidity density distributions at $\sqrt{s}=546 \mathrm{GeV}$ by 
TABLE II. - The cases possible to be solved

\begin{tabular}{lccccccc} 
& & & & & \multicolumn{3}{c}{ Remark } \\
\cline { 6 - 8 } & $\alpha$ & $\alpha^{\prime}$ & $\beta$ & $b$ & Model & $\sigma$ & $<K>$ \\
\hline Case A & 0 & 0 & 0 & $0,0.3$ & Model-0 & const & 0.5 \\
Case B & 0 & 0 & 0.056 & 0 & Model-0 & increasing & 0.5 \\
Case C & 0.105 & 0.105 & 0 & 0 & Model-1 & const & 0.5 \\
Case D & 0.105 & 0.210 & 0 & 0 & Model-2 & const & decreasing \\
\hline
\end{tabular}

the formulated models in Table 1 and by the simulation codes. We can see the following in the figure. $\left({ }^{1}\right)$

1) Difference of the densities, predicted by the simulation codes, is not negligible.

2) In the central region all simulation codes predict the rapidity densities consistent with the experimental data except HDPM.

3) In the middle rapidity region, i.e. $2.0 \leq \eta^{*} \leq 6.0$, which is the most important for the atmospheric diffusion of cosmic-ray particles, predictions by VENUS, QGSJET and DPMJET are higher than that of Model-2, while those by other codes are consistent with that of Model-2.

4) In the forward region all the codes predict consistent densities with that of Model-2.

5) The density distribution by UA5 code is almost consistent with that of Model- 2 over all rapidity region.

\section{3. - Air Showers of $E_{0}=10^{20} \mathrm{eV}$}

Our plan is to solve the diffusion of cosmic-ray particles in the atmosphere analytically on the basis of elementary processes, mentioned in Section 2, for the three models, listed in Table 1. $\left({ }^{2}\right)$ The diffusion equations can be solved for the cases, listed in Table 2, although it is not easy to solve them in a general way.

Then we obtain the size of the air shower, which is initiated by the incident proton with the fixed energy $E_{0}$, based on the solutions of diffusion equations. The size the air shower is defined as the number of charged particles which pass the horizontal plane at the observation level. The electron component is dominant among the charged particles in the air shower, and hence we refer only the electron number as the air shower size.

(i) Fig. 3 shows the transition curve of the air shower size for the primary proton with the energies $E_{0}=10^{18}, 10^{19}, 10^{20} \mathrm{eV}$ for Case A (with $b=0$ ), where the exact analytical solutions are possible. One can see in the figure that the air showers are at the maximum development at sea level and that the relation $E_{0} / N_{e} \simeq 2.0(\mathrm{GeV})$ holds approximatcly. (ii) Fig. 4 shows the ratio of the air shower size between the cases of $\mathrm{B}, \mathrm{C}, \mathrm{D}$ and the case A for the primary energy $E_{0}=10^{20} \mathrm{eV}$. One can see the following in the figure.

( $\left.{ }^{1}\right)$ The distributions by simulations are for NSD events while those by the formulation are for all inelastic events. The definitions of them are $\sigma_{N S D}=\sigma_{N D}+\sigma_{D D}$ and $\sigma_{i n e l}=\sigma_{N S D}+\sigma_{S D}$, where NSD, ND, DD and SD stand for non-single-diffractive, non-diffractive, double-diffractive and single-diffractive, respectively. According to the data by UA5 Collaboration[2] the pseudorapidity density of NSD events is higher by $10 \%$ than that of all inelastic events in the range $0 \geq \eta^{*} \geq 3.5$ and is almost equal in the region $\eta^{*} \geq 3.5$ at $\sqrt{s}=546 \mathrm{GeV}$.

$\left({ }^{2}\right) \pi \rightarrow \mu$ decay is neglected. 
(1) Effect of the charge exchange process of the surviving pion is almost constant over the atmospheric depth, amounting $13 \% .\left(^{3}\right)$

(2) Effect of increasing cross section is large $(100 \sim 200 \%)$ at high altitude, but is small $(\sim 18 \%)$ at sea level. $\left({ }^{3}\right)$.

(3) The effects of scaling violation, in Model-1 and in Model-2, have similar depth dependence, but the absolute values of them differ by five times.

(4) Model-2 gives smaller air shower size, and the attenuation of the air shower size after the shower maximum is very slow due to the small value of inelasticity. $\left({ }^{3}\right)$.

(5) At sea level the air shower size is dependent most strongly on the energy distribution of produced particles, but less strongly on the increasing cross section and on the charge exchange.

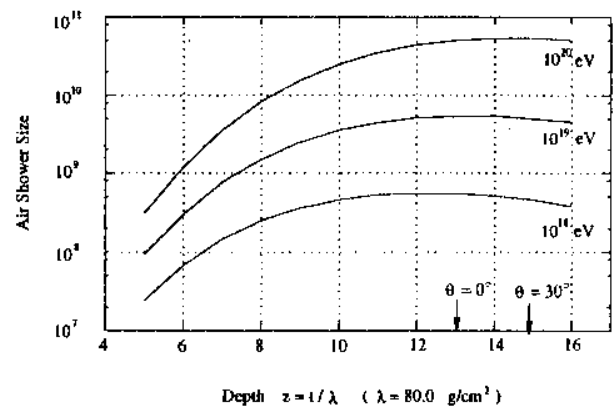

Fig. 3, - Transition curve of the air shower size for the primary proton with the energy $E_{0}=10^{18}, 10^{19}$, and $10^{20} \mathrm{eV}$, for Case A (Model-0, constant cross section and $b=$ $0)$. . The arrows indicate the depth of the sea level $\left(1,030 \mathrm{~g} / \mathrm{cm}^{2}\right)$ for the air showers with the inclination $\theta=0^{\circ}$ and $30^{\circ}$.

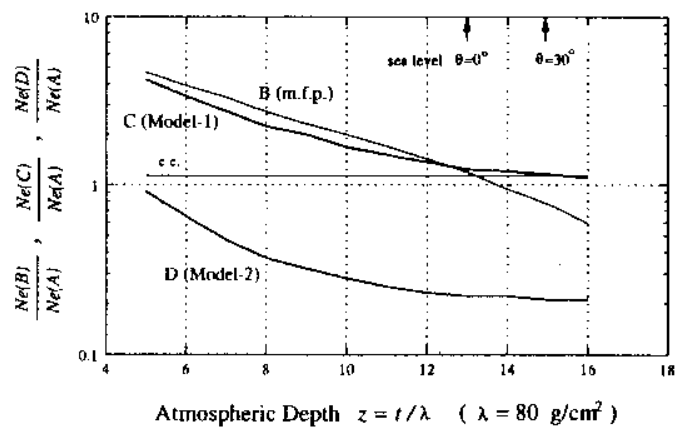

Fig. 4. - Ratio of air shower size, $N_{e}(\mathrm{~B}) / N_{e}(\mathrm{~A})$, $N_{e}(\mathrm{C}) / N_{e}(\mathrm{~A})$ and $N_{e}(\mathrm{D}) / N_{e}(\mathrm{~A})$, along the depth. The cases of $A, B, C$ and $D$ are tabulated in Table 2. The primary energy of a proton is $10^{20} \mathrm{eV}$.

(iii) The air shower size at sea level, expected by the present calculation, is tabulated in Table 3 for the incident proton of $E_{0}=10^{20} \mathrm{eV}$. In the table the effects of the charge exchange process and the increasing cross section are obtained by Fig. 4. To calculate the expected air shower size, to which the effects of charge exchange probability and increasing cross section are included, we multiplied all the factors because the factors are near 1.0 .

(iv) M. Nagano et al. examined the method of energy determination of extremely high energy air showers, employed by AGASA experiment, by the simulation code of CORSIKA[8](with QGSJET code). And they reached the conclusion that the method works well for the highest energy air showers. [9] The simulation gives $N_{e}=5.5 \times 10^{10}$ for the proton-induced air showers of $E_{0}=10^{20} \mathrm{eV} .\left({ }^{4}\right)$ We can see the following points by comparing the value with the expected sizes in Table 3 .

(1) The value by the simulation is between those of Model-1 and Model-2. In this sense our calculation and the simulation are consistent each other, because we saw in Section 2 that the pseudo-rapidity density distribution by QGSJET code is between those

$\left({ }^{3}\right)$ This tendency can be explained by the analytic expression of air shower size.

( ${ }^{4}$ ) We obtained $N_{e}=5.5 \times 10^{10}$ for the proton-induced air showers of $E_{0}=10^{19} \mathrm{eV}$, from the figure in Ref.[9], and multiplied it by 10 . 
TABLE III. - Air shower size at sea level by the models for the incident proton of $E_{0}=10^{20} \mathrm{eV}$

\begin{tabular}{lcccc} 
& Model-0 & Model-1 & CORSIKA & Model-2 \\
\hline size $^{*}$ & $5.0 \times 10^{10}$ & $6.2 \times 10^{10}$ & & $1.1 \times 10^{10}$ \\
ratio to Model-0 & $(\times 1.0)$ & $(\times 1.23)$ & & $(\times 0.22)$ \\
charge exchange & $\times 1.13$ & $\times 1.13$ & & $\times 1.13$ \\
increasing cross section & $\times 1.18$ & $\times 1.18$ & & $\times 1.18$ \\
\hline size (expected) $^{* *}$ & $6.7 \times 10^{10}$ & $8.3 \times 10^{10}$ & $5.5 \times 10^{10}$ & $1.5 \times 10^{10}$ \\
\hline
\end{tabular}

* without the processes of increasing cross section and the charge exchange.

** with the processes of increasing cross section and the charge exchange.

by Model-1 and Model-2.

(2) If we take Model-1, the energy spectrum of highest energy air showers shifts to the left (toward lower energy) by a factor 1.5 .

(3) If we take Model-2, which is the best-fitted to the experimental data, the energy spectrum shifts to right (toward higher energy) by a factor 3.7 .

Among the major factors to govern the cosmic-ray diffusion in the atmosphere the encrgy distribution of produced particles, the charge exchange probability of the surviving pion and the increasing cross section of hadron-air collisions -, the first one has the largest effect on the size of extremely high energy air showers. Hence we have to specify the energy distribution of produced particles in multiple particle production in more detail, in order to confirm the extremely high energy cosmic rays exceeding GZK cut-off energy.

(v) The item (3) in the above paragraph (iv) makes the puzzle of extremely high energy cosmic rays more serious. Probably it is not irrelevant to conclude that the energy dependences of the scaling violation parametcrs in Model-2 are not valid at extremely high energy region. In other words Model-2 does not describe the extremely high energy air showers, although the model is formulated based on the experimental data of direct observation. This is due to the fact that Model-2 predicts quite small inelasticity at high energies. For example, the value is as small as 0.2 even at $E_{0}=10^{16} \mathrm{eV}$. (See Fig. 1.) According to our previous analysis of attenuation mean free paths of hadron and $(e, \gamma)$ components [10], the inelasticity of $\langle K\rangle=0.5$ is compatible but smaller inelasticity is not compatible with the experimental data in the energy region of $10^{14} \sim 10^{16} \mathrm{eV}$.

\section{REFERENCES}

[1] A. Ohsawa, Proc. XIth ISVHECRI (Campinas), to appear in Nucl. Phys. B (Proc. Suppl.).

[2] G.L. Alner et al. (UA5 Collaboration), Z. Phys. C33 (1986) 1; Nucl. Phys. B291 (1987) 445; Phys. Rep. Nos.5 and 6 (1987) 247.

[3] E. Pare et al., Phys Lett. B242 (1990) 531.

[4] R. Haar et al, Phys. Lett. B401 (1997) 176

[5] N. Arata, Nucl. Phys. B211 (1983) 189.

[6] R. Feynman, Phys. Rev. Lett. 23 (1969) 1415.

[7] F.E. Taylor et al., Phys. Rev. D14 (1976) 1217.

[8] References of the simulation codes are found in the paper by J. Knapp et al., Preprint of Forchungszentrum Karlsruhe, FZKA 5828 (1996).

[9] M, Nagano et al., Preprint of Forschungszentrum Karlsruhe, FZKA 6191 (1998).

[10] A. Ohsawa et al., Phys. Rev. D49 (1992) 3128-3133. 